\title{
Charakteristische Eigenschaften laserdeponierter Poly(methyl-methacrylat)-Filme
}

\author{
Dissertation \\ zur Erlangung des Doktorgrades der Mathematisch-Naturwissenschaftlichen \\ Fakultäten \\ der Georg-August-Universität zu Göttingen
}

Vorgelegt von

Erik Süske

aus Hameln

Göttingen 2005 
Referent:

Korreferent:

Tag der mündlichen Prüfung: 17. März 2005
Prof. Dr. H.-U. Krebs

Prof. Dr. R. Kirchheim 


\section{Inhaltsverzeichnis}

1 Einleitung 1

2 Schichtherstellung und Charakterisierungsmethoden 5

2.1 Herstellung der Proben . . . . . . . . . . . . . . . . . . . . . . 5

2.2 Targets und Substrate . . . . . . . . . . . . . . . . . 7

2.3 Untersuchung der Mikrostruktur . . . . . . . . . . . . . . . . . . . 9

2.4 Chemische Analyse der PMMA-Filme . . . . . . . . . . . . . . . . . 10

2.5 Charakterisierungsmethoden . . . . . . . . . . . . . 11

3 Ablation von PMMA 14

3.1 Chemische Modifikation des Targets . . . . . . . . . . . . . . . . . . . 14

3.2 Ablationsmechanismus und -produkte . . . . . . . . . . . . . . . 18

4 Deposition auf dem Substrat $\quad 24$

4.1 Mikrostruktur laserdeponierter Filme . . . . . . . . . . . . . . . . 24

4.2 Molmassenverteilung deponierter PMMA-Droplets . . . . . . . . . . . 31

4.3 Struktur und Zusammensetzung von PMMA-Filmen . . . . . . . . . . 35

4.4 Zusammensetzung der vernetzten Komponente . . . . . . . . . . . . . 39

4.5 Mechanische Eigenschaften . . . . . . . . . . . . . . . . . . . . . . 42

5 Einfluss der Energiedichte $\quad 46$

5.1 Molmassenverteilung von Droplets und Oligomeren . . . . . . . . . 46

5.2 Veränderung der chemischen Zusammensetzung . . . . . . . . . . . . 49

5.3 Modifikation der mechanischen Eigenschaften . . . . . . . . . . 51

5.4 PVCin: Verfestigung mit zunehmender Vernetzung . . . . . . . . . . . 51 
6 Einfluss der Substrattemperatur $\quad 56$

6.1 Veränderung der Oberfläche . . . . . . . . . . . . . . 56

6.2 Änderung der Molmasse . . . . . . . . . . . . . . . . . . . . . 58

6.3 Reaktionsmechanismus . . . . . . . . . . . . . . . . 59

6.4 Chemische Zusammensetzung der Filme . . . . . . . . . . . . . . . . 62

6.5 Thermische Zersetzung . . . . . . . . . . . . . . . . . 64

6.6 Mechanische Eigenschaften . . . . . . . . . . . . . . 66

7 Thermische Veränderung der Filme $\quad 70$

7.1 Glasübergang laserdeponierter Filme . . . . . . . . . . . . . . . . . . 70

7.2 Zerfall des Polymers . . . . . . . . . . . . . . . . . . . . 74

7.3 Vernetzte Komponente als Rückstand . . . . . . . . . . . . . . . . 77

7.4 Mechanische Eigenschaften . . . . . . . . . . . . . . . . . 78

7.5 Mechanische Spektroskopie . . . . . . . . . . . . . . . 80

$\begin{array}{llr}8 & \text { Zusammenfassung } & 86\end{array}$ 


\section{1}

\section{Einleitung}

Polymere Werkstoffe nehmen einen immer größeren Platz in unserer Umwelt ein. Ihre Verbreitung reicht von organischen Makromolekülen, wie sie zum Beispiel als Proteine, Polysaccharide oder Kautschuke in der Natur gefunden werden, bis zu synthetischen Werkstoffen, deren Produktionsmenge das Volumen des klassischen Werkstoffs Stahl erreicht [46]. Polymere werden technisch auf Grund ihrer chemischen Korrosionsbeständigkeit, ihrer einfachen Verarbeitung, ihrer mechanischen Eigenschaften wie Bruchfestigkeit und Zähigkeit und ihres geringen Gewichtes eingesetzt [12]. Anwendungsgebiete synthetischer Polymere reichen von einfachen Verpackungsmaterialien über Textilien, und optische Komponenten bis zu komplexen elektronischen Bauteilen. Entsprechend vielfältig sind auch die Eigenschaften der Polymere. Sie werden wesentlich bestimmt durch die Struktur sich wiederholender Monomereinheiten mit durchgehender Hauptkette und abzweigenden Nebengruppen, durch ihr Molekulargewicht und durch eine Vernetzung der Materialien.

Mit der Miniaturisierung von Bauelementen verbunden ist eine Entwicklung von dünnen Schichten, deren Bedeutung in den letzten Jahrzehnten stetig gewachsen ist. Auch Polymere werden vermehrt als dünne Filme eingesetzt. Die Anwendungen dieser Schichten sind vielfältig. Einfache Beispiele sind optische, mechanische oder korrosionshemmende Vergütungen, aber auch pharmazeutische, elektronische oder mikrosensorische Aufgabengebiete seien genannt [14].

Zur Herstellung dieser Schichten wurden die unterschiedlichsten Methoden entwickelt. Einfache Herstellungsverfahren basieren auf einem nasschemischen Prozess, bei dem das Polymer in Lösung auf entsprechende Substrate aufgetragen wird. Am 
weitesten verbreitet ist dabei das Spin-Coating-Verfahren. Für die weitere Verarbeitung von polymeren Dünnschichten kann die Verwendung von Lösungsmitteln von Nachteil sein: Einerseits ist eine gleichbleibende Schichtqualität stark vom Verhalten des Lösungsmittels abhängig, andererseits sind komplexere Depositionsvorgänge wie die Herstellung von Mehrfachschichten unterschiedlicher Materialien wegen des Abdampfens des Lösungsmittels mit langen Verzögerungen verbunden. Daher wurden alternative Verfahren entwickelt, bei denen die Schichten lösungsmittelfrei aufgebracht werden. Ein häufig verwendetes Verfahren ist die Plasmapolymerisation [5], bei der die Schicht durch chemische Reaktionen von Monomeren innerhalb eines Niederdruckplasma auf dem Substrat entsteht. Ein Nachteil dieser Methode ist die Anwendbarkeit auf nur wenige Polymere und der hohe Vernetzungsgrad der Schichten, der für viele Einsätze unerwünscht ist.

Eine andere Herstellungsmethode ist die gepulste Laserdeposition (pulsed laser deposition, PLD), die bereits 1965 erfolgreich zur Herstellung von organischen Materialien eingesetzt werden konnte [81]. Der Durchbruch dieser Depositionsmethode ist eng mit der erfolgreichen Herstellung von hochtemperatursupraleitenden Filmen im Jahr 1987 [21] - vorwiegend $\mathrm{YBa}_{2} \mathrm{Cu}_{3} \mathrm{O}_{7}$ - verbunden. Seitdem hat die gepulste Laserdeposition ihre Vielseitigkeit bei der Herstellung verschiedener Materialien unter Beweis gestellt. Die Vorteile der gepulsten Laserdeposition - beispielsweise bei Chrisey und Hubler [13] zusammenfassend dargestellt - liegen neben der einfachen technischen Umsetzung mit einer Energiequelle außerhalb des Vakuums der Depositionsanlage vor allem im stöchiometrischen Übertrag, der die Deposition fast beliebiger Materialien erlaubt. Entsprechend konnten Hansen und Robitaille 1988 [41, 42] die Möglichkeit der gepulsten Laserdeposition unterschiedlichster Polymere vorstellen - darunter auch Poly(methyl-methacrylat), PMMA.

PMMA, das seit 1933 synthetisch hergestellt werden kann, ist ein glasklares Thermoplast, das - anwendungsbezogen - auch als Plexiglas bekannt ist. Technische Anwendungen von PMMA-Dünnfilmen sind beispielweise Schutzschichten gegen Elektronenstrahlung. Dünne Schichten werden aber auch in medizinischen Aufgabenbereichen, Halbleiter-Design und in der Lithographie als Photoresist [64] eingesetzt.

Für eine Beschreibung der Deposition, wie sie in dieser Arbeit entwickelt werden soll, ist vor allem die letzte Anwendung mit großen Vorteilen verbunden: Wegen 
der Anforderung immer kleinerer lithographischer Strukturen wurde die Wechselwirkung von PMMA mit UV-Strahlen detailliert untersucht. Ein besonderer Schwerpunkt lag dabei auf dem Abtragsprozess, der ausführlich charakterisiert wurde. Das große Interesse an diesem Prozess lässt sich daraus ermessen, dass 1992 über 40\% aller Veröffentlichungen über die gepulste Laserdeposition von Polymeren sich mit PMMA beschäftigt haben [86]. Trotz dieser vielfältigen Untersuchungen steht eine zusammenhängende Beschreibung der Mechanismen der gepulsten Laserdeposition von PMMA noch aus.

In dieser Arbeit sollen daher die Depositionsmechanismen von PMMA unter Verwendung eines KrF-Excimerlasers mit einer Wellenlänge von $248 \mathrm{~nm}$ erarbeitet werden. Dazu gehört eine vollständige Charakterisierung der Schichten - von der Zusammensetzung und molekularen Masse der Filme über die Mikrostruktur bis zu makroskopischen mechanischen Eigenschaften. Die Beschreibung der Deposition soll insbesondere Ablationsvorgänge mit einschließen und die Deposition mit den Eigenschaften der Filme verbinden. Im Hinblick auf einen späteren Einsatz der Filme von Interesse ist - neben einem Vergleich der Depositionsmethode mit anderen Verfahren - eine Möglichkeit, die Eigenschaften der Filme einstellen zu können. Über ein Verständnis der maßgeblichen Mechanismen soll diese Modifikation der Filme durch eine Veränderung der Depositionsparameter abgeleitet werden. Anwendungsbezogen sind auch Untersuchungen hinsichtlich der thermischen Stabilität und Alterung laserdeponierter PMMA-Filme.

Der experimentelle Teil dieser Arbeit gliedert sich daher in drei Teile. Die Kapitel 3 und 4 beschreiben die Ablation und Deposition der Filme im Hinblick auf Filmcharakteristika. Im nächsten Teil, der die Kapitel 5 und 6 umfasst, werden Modifikationen der Filme über einen Eingriff in den Ablationsmechanismus einerseits und den Depositionsmechanismus andererseits beschrieben. Im Kapitel 7 werden die thermischen Veränderungen der Filme bei einer Auslagerung diskutiert.

Der Prozess der Ablation ist der wesentliche Schlüssel zum Verständnis der Eigenschaften laserdeponierter PMMA-Schichten. Ihm wird im Rahmen einer ausführlichen Literaturdiskussion im Kapitel 3 besonderer Platz eingeräumt. Eigene Experimente zum Ablationsprozess werden mit den aus der Literatur gezogenen Schlüssen über Ablationsmechanismen verglichen.

Der eigentliche Depositionsprozess findet nach dem Übertrag der Ablationspro- 
dukte auf das Substrat statt. Im Kapitel 4 wird dieser Prozess aus den Eigenschaften der Filme - angefangen von der Mikrostruktur über die Molmasse bis zur chemischen Zusammensetzung - entwickelt. Besondere Berücksichtigung finden auch makroskopische Eigenschaften wie der Elastizitätsmodul.

Aus dem Ablations- und Depositionsmechanismus lassen sich Möglichkeiten ableiten, die Eigenschaften der Filme über die Zusammensetzung der Komponenten zu verändern. Im Kapitel 5 wird mit der Variation der Energiedichte ein Eingriff in den Ablationsprozess vorgeschlagen. Besonderer Wert wird dabei auf eine Charakterisierung der Änderung der mechanischen Eigenschaften gelegt, die mit einem Modellsystem - Poly(vinyl-zimtsäureester), PVCin, verglichen werden.

Eine andere Möglichkeit, die Schichteigenschaften zu verändern, bietet eine Modifikation der Ablationsprodukte auf dem Substrat. Im Kapitel 6 wird der Einfluss einer zunehmenden Substrattemperatur auf die Filme charakterisiert.

Über Alterungsprozesse bieten Auslagerungen von Polymeren eine Möglichkeit, nachträglich die Eigenschaften der Materialien zu beeinflussen. Im Kapitel 7 werden diese Veränderungen bei einer thermischen Auslagerung diskutiert. Ausführlich werden Relaxationen - wie der kalorische Glasübergang - der Filme diskutiert, bevor der thermische Zersetzungsprozess detailliert charakterisiert wird. Als Anwendung wird der Einfluss von Relaxationen und Zersetzung auf die mechanischen Eigenschaften der Filme beschrieben.

Abschließend werden die Ergebnisse in Kapitel 8 zusammengefasst. 


\section{2}

\section{Schichtherstellung und}

\section{Charakterisierungsmethoden}

Im Rahmen dieser Arbeit wurden Polymerschichten mit Hilfe der gepulsten Laserdeposition (Pulsed Laser Deposition, PLD) hergestellt und anschließend charakterisiert. In diesem Kapitel werden zunächst die Grundzüge der Herstellungsmethode kurz skizziert. Im Folgenden wird das als Targetmaterial verwendete Poly(methylmethacrylat), PMMA, vorgestellt, bevor die experimentellen Methoden, mit denen die Filme auf ihre Struktur und Eigenschaften untersucht wurden, beschrieben werden.

\subsection{Herstellung der Proben mit gepulster Laserdeposition}

Die gepulste Laserdeposition hat sich zu einem Standardverfahren zur Herstellung von dünnen Schichten etabliert. Eine ausführliche Diskussion dieser Herstellungsmethode ist zum Beispiel bei Chrisey und Hubler [13] zu finden. Der hier verwendete Aufbau ist in Abbildung 2.1 skizziert. Zur Herstellung der Polymerschichten wurde ein gepulster KrF-Excimerlaser (Lambda Physik LPX 110i) mit einer Wellenlänge von $248 \mathrm{~nm}$, einer Pulsdauer von 30 ns bei einer Frequenz von $5 \mathrm{~Hz}$ verwendet. Der Laserstrahl, der eine maximale Laserenergie von $300 \mathrm{~mJ}$ aufweisen kann, wird mit Hilfe einer Linse unter einem Winkel von $45^{\circ}$ auf ein Polymertarget fokussiert, das 


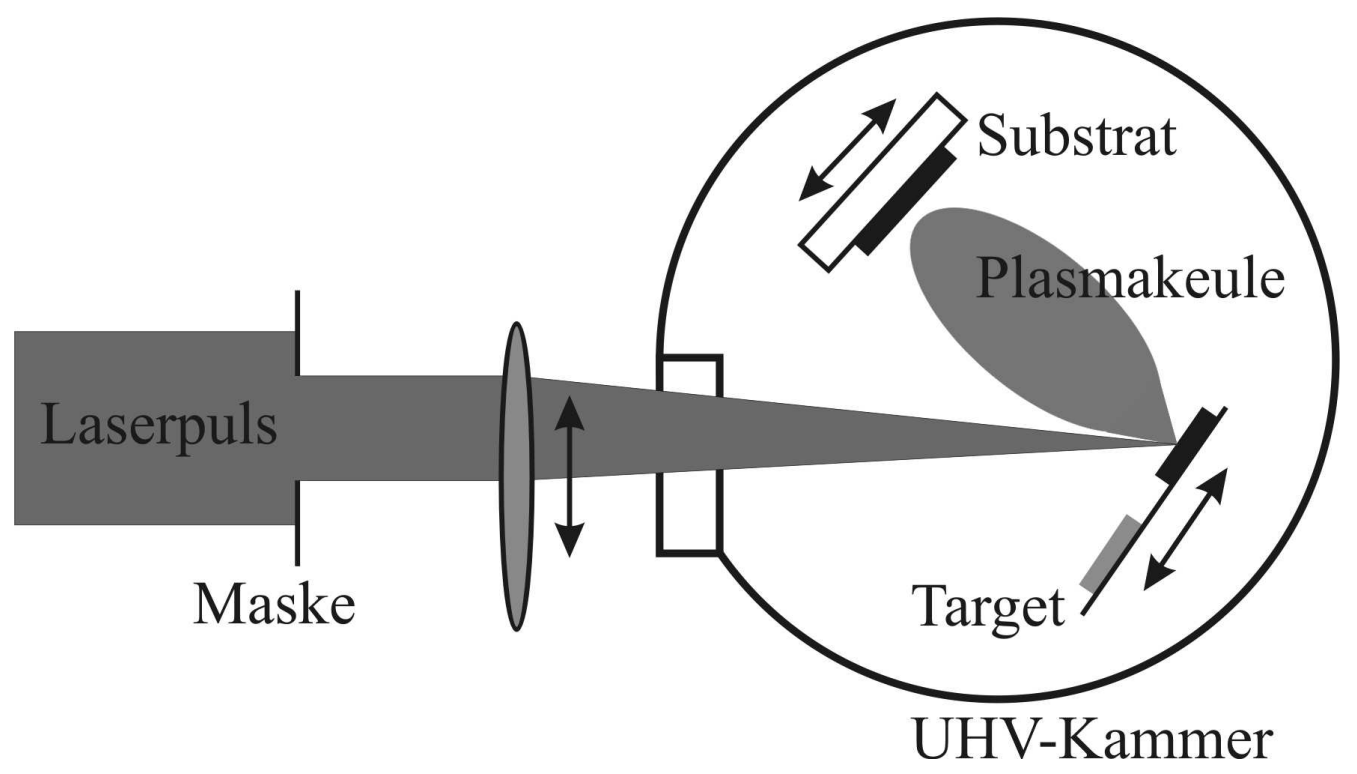

Abbildung 2.1: Prinzip der gepulsten Laserdeposition.

sich in einer Vakuumkammer mit einem Restgasdruck von weniger als $1 \cdot 10^{-8} \mathrm{mbar}$ befindet. Der Brennfleck auf dem Target weist eine Größe von $6 \mathrm{~mm}^{2}$ auf; durch eine geeignete Wahl der Laserenergie in Kombination mit Abschwächern können so auf dem Target mittlere Energiedichten von $0,1-5 \mathrm{~J} / \mathrm{cm}^{2}$ eingestellt werden. Die Depositionsanlage, die während der Schichtherstellung computergesteuert wird, ist detailliert von Fähler [29] beschrieben worden.

Bei Energiedichten oberhalb der Ablationsschwelle (PMMA: 65-100 mJ/ $\mathrm{cm}^{2}$ $[83,9])$ wird beim Depositionsprozess durch den Laserstrahl Material vom Target abgetragen (Ablation), und annähernd senkrecht von der Targetoberfläche weg auf ein heizbares Substrat in einem Abstand von 30 - 40 mm deponiert; die Aufwachsrate von PMMA-Filmen liegt bei der Deposition auf ein Substrat mit einer Temperatur von $25^{\circ} \mathrm{C}$ zwischen 0,1 und $0,5 \mathrm{~nm} /$ Puls [91].

Während der Deposition kann der Partialdruck einzelner Moleküle mit einer Masse von bis zu $300 \mathrm{~g} / \mathrm{mol}$ und der Gesamtdruck in der UHV-Kammer mit einem Quadrupol-Massenspektrometer (VG IQ Smart ${ }^{+}$) bestimmt werden. Die Analyse der Ablationsprodukte erlaubt eine Kontrolle der Ablation und Depositionsrate [61]. 


\subsection{Targets und Substrate}

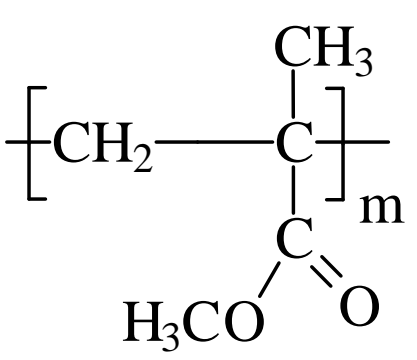

Abbildung 2.2: Strukturformel von PMMA.
Als Targetmaterial wurde überwiegend pulverförmiges PMMA mit definierter Molmasse $\left(\mathrm{M}_{W}=996,350\right.$ bzw. $\left.15 \mathrm{~kg} / \mathrm{mol}\right)$ verwendet, das bei Aldrich bezogen wurde. Abbildung 2.2 skizziert die Strukturformel des Makromoleküls. Zur Herstellung fester Targets wurde das Material ohne Hinzugabe von Lösungsmitteln unter einem Druck von $3 \cdot 10^{7} \mathrm{~N} / \mathrm{cm}^{2}$ für mindestens eine Stunde bei etwa $120{ }^{\circ} \mathrm{C}$ gepresst. In Einzelfällen wurden auch kommerziell erhältliche PMMA-Folien von klinischer Reinheit, die über Goodfellow bezogen wurden, verwendet. PMMA

mit geringen Molmassen $(<5000 \mathrm{~g} / \mathrm{mol})$ wurde in Zusammenarbeit mit dem Institut für Physikalische Chemie der Universität Göttingen durch radikalische Polymerisation von Methylmethacrylat (MMA) in Toluol hergestellt (siehe Abbildung 2.3). Als Initiator wurde AIBN $\left(\mathrm{NCCH}_{2} \mathrm{~N}=\mathrm{NCH}_{2} \mathrm{CN}\right)$ verwendet, das bei Erwärmung auf über $80^{\circ} \mathrm{C}$ in Starterradikale zerfällt, die die Polymerisationsreaktion einleiten. Geringe Molmassen wurden durch Zugabe von DDM $\left(\mathrm{CH}_{3}\left(\mathrm{CH}_{2}\right)_{11} \mathrm{SH}\right)$ als Regler in unterschiedlichen Konzentrationen realisiert. Nach Beendigung der Polymerisationsreaktion wurde das Toluol entfernt und das pulverförmige PMMA unter Druck zu festen Targets gepresst.

Eine Reinigung der Targets wurde auf kurzes Abspülen mit Methanol beschränkt. Auf Grund der schwachen Absorption von 248 nm-Strahlung zeigt PMMA Inkubationseffekte, durch die die Abtragsrate erst nach 40 Pulsen konstant ist [85]. Um Einflüsse dieser Effekte auf die Schichteigenschaften zu minimieren, werden die Targets vor einem Depositionsvorgang mit 50 Pulsen/Position gereinigt.

Als Substrate dienten Si-Einkristalle, deren (111)-Orientierung um $5^{\circ}$ aus der Probennormalen verkippt ist. Die Kristalle sind mechanisch poliert und werden chemisch mit Azeton und Methanol im Ultraschallbad gereinigt und mit warmer Luft getrocknet. Der Einbau der Substrate in die UHV-Kammer erfolgte über eine Schleuse, so dass das Vakuum nicht gebrochen werden musste. Dadurch wurden 


\section{Starterreaktion}

$$
\mathrm{NC}-\mathrm{CH}_{2}-\mathrm{N}=\mathrm{N}-\mathrm{CH}_{2}-\mathrm{CN} \stackrel{\mathrm{T}}{\longrightarrow} 2 \mathrm{NC}-\mathrm{CH}_{2} \bullet+\mathrm{N}_{2}
$$

2. Initierung<smiles>C=C(C)C(=O)OCCCC#N</smiles>

3. Propagation<smiles>[R]CC(C)(C[C@@](C)(CC)C(=O)OC)C(=O)OC</smiles>

4. Terminierung

$$
\begin{aligned}
& \mathrm{RM}_{\mathrm{n}}^{\bullet}+\mathrm{RM}_{\mathrm{m}}^{\bullet} \longrightarrow \mathrm{RM}_{\mathrm{m}+\mathrm{n}} \mathrm{R}
\end{aligned}
$$

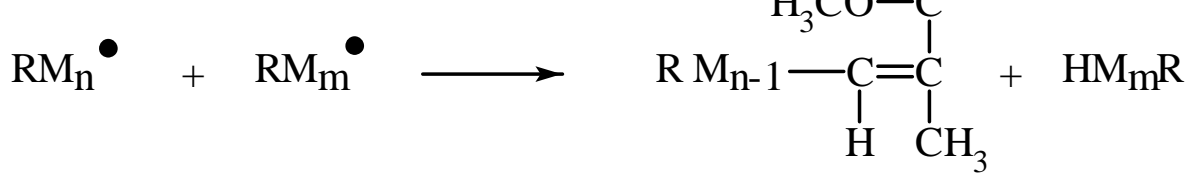

Abbildung 2.3: Schema der radikalischen Polymerisation von PMMA. Durchgeführt wurde die Polymerisation zur Herstellung von niedermolekularem PMMA mit $20 \mathrm{ml}$ MMA und 0,2 g AIBN $\left(\mathrm{NCCH}_{2} \mathrm{~N}=\mathrm{NCH}_{2} \mathrm{CN}\right)$ als Starter in $50 \mathrm{ml}$ Toluol und Hinzugabe unterschiedlicher DDM-Konzentrationen $\left(\mathrm{CH}_{3}-\left(\mathrm{CH}_{2}\right)_{11}-\mathrm{SH}\right)$ als Regler bei Temperaturen zwischen 80 und $100{ }^{\circ} \mathrm{C}$. 


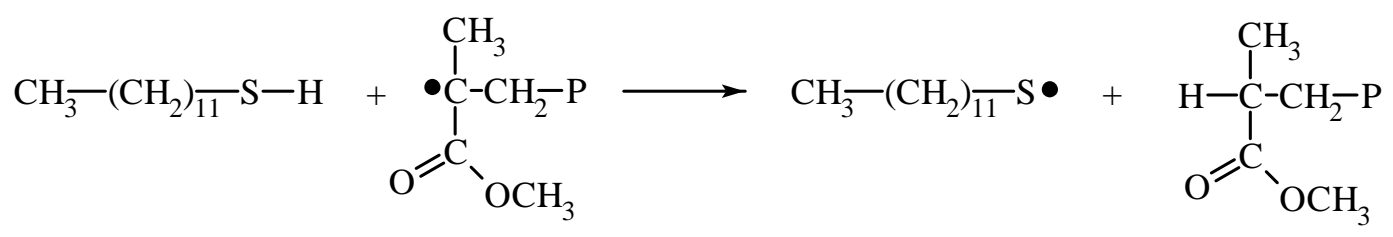<smiles>C=C(C)C(=O)OC</smiles>

Abbildung 2.4: Abbruch der Propagation durch Reaktion mit DDM $\left(\mathrm{CH}_{3}-\right.$ $\left.\left(\mathrm{CH}_{2}\right)_{11}-\mathrm{SH}\right)$ als Regler.

Adsorbate auf den Targets minimiert und ähnliche Bedingungen für mehrere Depositionen erreicht. Zur Analyse der chemischen Zusammensetzung mit Hilfe der Infrarotspektroskopie (vgl. Kap. 2.4.1) wurden wegen der hohen Reflektivität vereinzelt Al-Substrate verwendet, bei Transmissionsmessungen (vgl. Kap. 2.4.2) auch Quarzglassubstrate.

\subsection{Untersuchung der Mikrostruktur}

\subsubsection{Rasterelektronenmikroskopie (REM)}

Die Oberfläche der PMMA-Filme wurde mit Hilfe eines Rasterelektronenmikroskops vom Typ Cambridge Instruments S360 (W-Kathode, 20 kV, Sekundärelektronendetektor) untersucht. Grundzüge der Rasterelektronenmikroskopie (REM) sind zum Beispiel bei Lüth [67] beschrieben. Um starke Aufladungserscheinungen der elektrisch isolierenden Polymerschichten zu vermeiden, wurden polymere Proben mit einer 15 bis $20 \mathrm{~nm}$ dicken Au-Schicht überzogen. 


\subsubsection{Rasterkraftmikroskopie (AFM)}

Zusätzliche Höheninformationen lassen sich mit Hilfe der Rasterkraftmikroskopie (atomic force microscopy, AFM) [6] gewinnen. Dazu wurden die Proben in Zusammenarbeit mit dem I. Physikalischen Institut, Universität Göttingen, mit einem NanoScope III im Taping-Mode analysiert. Zur Bildauswertung wurde auf die Software NanoScope R III, Version 5.12r3 zurückgegriffen.

\subsubsection{Röntgenstreuung (XRD) und -reflektometrie (SAXS)}

Zur Analyse der Mikrostruktur der Filme wurde auf die Röntgenstreuung (X-ray diffraction, XRD), die zum Beispiel bei Cullity [17] ausführlich beschrieben ist, zurückgegriffen. Verwendet wurde ein Philips-4-Kreisdiffraktometer vom Typ X'Pert MRD mit einer Co-Röhre in Verbindung mit einem Fe-Filter (Wellenlänge Co- $K_{\alpha}$ $1,7902 \mathrm{~nm})$.

Gleichzeitig lassen sich dünne PMMA-Filme (Schichtdicke $<50 \mathrm{~nm}$ ) mit Hilfe der Röntgenreflektometrie (small angle X-ray scattering, SAXS) [72, 36] charakterisieren. Eine Anpassung der gemessenen Spektren wurde mit Hilfe des IMDSoftwarepakets [103] vorgenommen, das auf einem von Parratt [75] entwickelten Rekursionsverfahren beruht.

\subsection{Chemische Analyse der PMMA-Filme}

\subsubsection{Fouriertransformationsinfrarotspektroskopie (FTIR)}

Zur Analyse der chemischen Zusammensetzung der Polymerfilme wurde auf die Fouriertransformationsinfrarotspektroskopie (FTIR) zurückgegriffen, die zum Beispiel bei Günzler [38] detailliert beschrieben wird. Im Rahmen dieser Arbeit wurde das FTIR-Absorptions-Reflexions-Verfahren (FTIRAR) mit einem Bruker Tensor 35 mit MCT-Detektor in Verbindung mit einer Reflexionseinheit mit variablem Einfallswinkel oder - in Zusammenarbeit mit dem Institut für Rechtsmdizin, Universität Göttingen - ein Nicholet 710 mit einem Plan-Mikroskop verwendet; soweit nicht anders angeben, wurde der Einfallswinkel bei $5^{\circ}$ konstant gehalten. Aufgenommen wurden jeweils 100 Scans mit einer Auflösung von $4 \mathrm{~cm}^{-1}$, die Transmission wurde 
als Quotient aus dem Spektrum des Filmes auf dem Substrat und dem Spektrum eines leeren Substrates angegeben.

\subsubsection{Transmissionsmessungen}

In Zusammenarbeit mit dem Laserlaboratorium Göttingen e.V. wurde mit Hilfe der UV-VIS-Spektrometrie die optische Transmission der auf Quarzglassubstraten deponierten Filme charakterisiert. Verwendet wurde ein Perkin-Elmer Lambda 19 Spektrometer im Wellenlängenbereich von 200 - 800 nm. Analog zur FTIR-Spektroskopie wurde die Transmission als Verhältnis aus dem Spektrum von Film und Substrat einerseits und dem Spektrum des leeren Spektrometers andererseits angeben.

\subsubsection{Größenauschlusschromatographie (SEC)}

Zur Bestimmung der Molmasse der deponierten PMMA-Schichten wurde in Zusammenarbeit mit dem Institut für Physikalische Chemie, Universität Göttingen, die Größen-Ausschluss-Chromatographie (size exclusion chromatography, SEC) angewendet, bei der Trennung der gelösten Moleküle durch sterischen Ausschluss in einem porösen Trenngel beruht [12]. Dazu wurde ein Waters-System mit drei PSSSDV-Säulen mit einer Partikelgröße von $5 \mu \mathrm{m}$ und Porendurchmessern von $10^{4}, 10^{2}$ und $10 \mathrm{~nm}$ mit Tetrahydrofuran (THF) als Eluent mit einer Durchflussmenge von $1 \mathrm{ml} / \mathrm{min}$ verwendet, das mit PMMA-Standards (Polymer Standards Service, PSS, $800 \mathrm{~g} / \mathrm{mol}<\mathrm{M}_{P}<1,8 \cdot 10^{6} \mathrm{~g} / \mathrm{mol}$ ) kalibriert wurde. Die laserdeponierten PMMASchichten wurden durch Behandlung in THF (Raumtemperatur, mindestens eine Stunde) aufgelöst.

\subsection{Charakterisierungsmethoden thermischer und mechanischer Eigenschaften}

\subsubsection{Thermogravimetrische Analyse (TGA)}

Die thermische Stabilität und der Zerfall von Polymeren lassen sich mit Hilfe der thermogravimetrischen Analyse (TGA) aus dem Gewichtsverlust während eines Aufheizprozesses untersuchen. Dazu wurde die Probe in einer Perkin Elmer TGA 7 unter 
Argon (Reinheit 99,998\%) mit einer Temperaturrampe von $20 \mathrm{~K} / \mathrm{min}$ erhitzt und gleichzeitig die Masse mit einer minimalen Auflösung von 1,5 $\mu \mathrm{g}$ bestimmt.

\subsubsection{Ultramikrohärteprüfer}

Mechanische Eigenschaften wie die Härte dünner Schichten lassen sich mit Ultramikrohärteprüfern untersuchen [2, 74, 3, 4]. Verwendet wurde ein Fischer HV 100 C XYm oder - in Zusammenarbeit mit dem II. Physikalischen Institut der Universität Göttingen - ein Fischer HV 100 C XYProg, bestückt mit einer Diamantpyramide mit einem Öffnungswinkel von $136^{\circ}$. Zur Messung der Härte wird diese mit definierter Kraft bei gleichzeitiger Messung der Eindringtiefe in den Film gedrückt. Aus der Eindringtiefe bei maximaler Kraft ergibt sich die Universalhärte als $H U=1 / 26,429 \cdot F_{\max } / h^{2}$ [4]. Gleichzeitig lässt sich aus der plastischen Eindringtiefe nach Rücknahme der Kraft ein Elastizitätsmodul $E_{\mathrm{HU}}$ angeben [74, 78], der proportional zum klassischen Elastizitätsmodul $E$ ist $\left(E_{\mathrm{HU}}=1,03 \cdot E\right)$. Die Bestimmung der Materialkonstanten eines dünnen Filmes mit einem Mikrohärteprüfer setzt eine Dicke voraus, die mindestens der zehnfachen Eindringtiefe entspricht, um Einflüsse des Substrates vernachlässigen zu können. Experimentell wurden daher Filme mit einer minimalen Dicke von $15 \mu \mathrm{m}$ verwendet.

\subsubsection{Mechanische Spektroskopie}

Dynamische mechanische Eigenschaften lassen sich mit Hilfe der mechanischen Spektroskopie untersuchen [100]. Experimentell wurde dazu in Zusammenarbeit mit dem I. Physikalischen Institut, Universität Göttingen, auf einen Doppelpaddeloszillator (DPO) zurückgegriffen. Die auf einkristallinen Si-Substraten deponierten PMMAFilme werden zu Schwingungen mit einer Frequenz von etwa 5,4 kHz angeregt, und die Dämpfung dieser Schwingungen, die von den Eigenschaften und der Dynamik im Film abhängt, als Funktion der Temperatur bestimmt. Mechanische Eigenschaften der Filme wie der Schermodul $G$ können bei bekannten Eigenschaften des Substrates aus der Frequenzverschiebung der freien Schwingung über

$$
G_{\text {Film }}=\left(\frac{\Delta f}{f}+\frac{\rho_{\text {Film }} d_{\text {Film }}}{2 \rho_{\mathrm{Si}} d_{\mathrm{Si}}}\right) \cdot \frac{2 G_{\mathrm{Si}} d_{\mathrm{Si}}}{3 d_{\mathrm{Film}}}
$$


bestimmt werden. Eine detaillierte Beschreibung der verwendeten Anlage sowie eine Herleitung des mathematischen Formalismus findet sich bei Rösner [76].

\subsubsection{Dielektrische Spektroskopie}

Eine andere Methode zur Untersuchung von Relaxationen in Polymerfilmen bietet die dielektrischen Spektroskopie [47], bei der dielektrische Verlustspektren durch kapazitive Anregung der Probe gewonnen werden können. Diese Untersuchungen wurden in Zusammenarbeit mit dem I. Physikalischen Institut der Universität Göttingen und dem Lehrstuhl für Experimentalphysik V, Universität Augsburg, an einem Novocontrol $\alpha$-Analyzer vorgenommen. Die PMMA-Filme wurden mittels gepulster Laserdeposition auf $\mathrm{Cu}$-Substraten aufgebracht. Auf die Filme wurde als Gegenelektrode eine $\mathrm{Cu}$-Schicht aufgebracht. Zum Vergleich wurden auch dielektrische Verlustspektren vom Targetmaterial, das beidseitig mit Cu-Elektroden beschichtet wurde, bestimmt. 


\section{3}

\section{Ablation von PMMA}

Die Eigenschaften laserdeponierter PMMA-Filme hängen wesentlich vom Ablationsprozess ab, der aus zwei unterschiedlichen Prozessen, die nacheinander ablaufen, besteht: Das Targetmaterial wird durch Wechselwirkungen mit Laserbestrahlung verändert, bevor ein Abtrag von Material beobachtet wird. In diesem Kapitel werden diese in der Literatur beschriebenen Prozesse ausführlich diskutiert und mit eigenen Ergebnissen verglichen.

\subsection{Chemische Modifikation des Targets}

Als Wechselwirkung zwischen PMMA und Laserpuls sind unterschiedliche Prozesse möglich: Die Energie der einfallenden Lichtquanten beträgt bei einer Wellenlänge von $248 \mathrm{~nm}$ etwa $5 \mathrm{eV}$. Diese Energie liegt oberhalb der Bindungsenergien von kovalenten Einfachbindungen mit einer typischen Energie von $4 \mathrm{eV}$ (vgl. Tab. 3.1). Damit ist es grundsätzlich möglich, die Energie der Lichtquanten auf die Bindungen zu übertragen und unmittelbar eine Bindung zu brechen. Hinweise für diesen photochemischen Mechanismus werden in der Modifikation von Polymeren ohne Ablation (Inkubation) gefunden [58]. Als zweite Möglichkeit bietet sich ein photothermischer Prozess an, bei dem die Energie im Material in Wärme umgewandelt wird (subsurface heating) [70], und dadurch das Polymer zersetzt wird. Anzeichen für diesen Prozess können vor allem in den Ablationsprodukten gefunden werden, die häufig den Produkten der thermischen Pyrolyse entsprechen [9] oder thermische Energien aufweisen [18, 97]. 


\begin{tabular}{ll}
\hline Bindung & Bindungsenergie $(\mathrm{eV})$ \\
\hline$\left(\mathrm{CH}_{2}\right)_{2}\left(\mathrm{CH}_{3}\right) \mathbf{C}-\mathrm{COOCH}_{3}$ & $3,1-3,6$ \\
$\left(\mathrm{CH}_{2}\right)_{2}\left(\mathrm{CH}_{3}\right)\left(\mathrm{COOCH}_{3}\right) \mathbf{C}-\mathrm{CH}_{2}$ & $3,3-3,4$ \\
$\left(\mathrm{CH}_{2}\right)_{2}\left(\mathrm{COOCH}_{3}\right) \mathbf{C}-\mathrm{CH}_{3}$ & $3,4-3,5$ \\
$\left(\mathrm{CH}_{2}\right)_{2}\left(\mathrm{CH}_{3}\right) \mathrm{CCO}-\mathrm{OCH}_{3}$ & 4,1 \\
$\mathrm{C}=\mathrm{O}$ & 7,3 \\
\hline
\end{tabular}

Tabelle 3.1: Bindungsenergien von PMMA nach Manring [68]. Zum Vergleich ist die Energie einer $\mathrm{C}=\mathrm{O}-$ Bindung mit aufgeführt [71].

Beide Mechanismen werden als Wechselwirkung zwischen UV-Lasern und Polymeren in der Literatur bereits seit 20 Jahren kontrovers diskutiert. Eine aktuelle Zusammenfassung dieser Diskussionen bieten zum Beispiel Dyer [23] oder Chrisey et al. [14]. Abhängig von der jeweiligen Absorption der Polymere, der Wellenlänge und der Energiedichte des einfallenden Laserstrahls kann einer der beiden Mechanismen überwiegen. Bei der Deposition von PMMA kann keine eindeutige Aussage zu Gunsten eines der beiden Mechanismen getroffen werden: Photochemische Mechanismen scheinen bei der Modifikation von PMMA während der Inkubation eine bedeutende Rolle zu spielen [58], wohingegen der spätere Zerfall des Polymers über einen thermischen Prozess erklärt werden kann [11].

Zur Charakterisierung der Modifikationen des Targetmaterials infolge von Wechselwirkungen zwischen PMMA und Laser bieten sich Experimente an, in denen PMMA mit UV-Pulsen niedriger Intensität bestrahlt wird. Während dieser Bestrahlung verändert sich das Polymer, ohne dass ein nennenswerter Abtrag stattfindet [85]. Durch die Wechselwirkung mit $248 \mathrm{~nm}$-Laserpulsen niedriger Intensität $\left(40 \mathrm{~mJ} / \mathrm{cm}^{2}\right)$ wird das Polymer jedoch modifiziert: Küper und Stuke [58] beschreiben eine Absorptionszunahme vom UV-Bereich bis in den sichtbaren Wellenlängenbereich hinein. Die Transmission bei $248 \mathrm{~nm}$ sinkt auf 6\% des Ausgangswertes bei einer Bestrahlung mit 1000 Pulsen einer Energiedichte von $40 \mathrm{~mJ} / \mathrm{cm}^{2}$. Verbunden mit diesen Änderungen sind chemische Reaktionen des PMMA unter Bildung von Doppelbindungen. Unter ähnlichen experimentellen Bedingungen $\left(35 \mathrm{~mJ} / \mathrm{cm}^{2}\right)$ durchgeführte Experimente von Blanchet [11] zeigen gleichzeitig eine Reduktion der Molmasse von $5 \cdot 10^{5} \mathrm{~g} / \mathrm{mol}$ auf etwa $8 \cdot 10^{3} \mathrm{~g} / \mathrm{mol}$ nach 900 Pulsen. Als Mechanismus ist ein Zerfall vorstellbar, der durch die photochemische Abspaltung der Ne- 
bengruppe $\mathrm{COOCH}_{3}[59,64]$ oder Teilen davon initiiert wird. Abbildung 3.1 zeigt einen von Gupta et al. [39] vorgeschlagenen photochemischen Zerfallsprozess, bei dem als unterschiedliche Reaktionsprodukte $\mathrm{CO}, \mathrm{CO}_{2}, \cdot \mathrm{CH}_{3}, \cdot \mathrm{OCH}_{3}, \cdot \mathrm{COOCH}_{3}$ mit teilweise radikalischem Charakter und $-\mathrm{CH}_{2}-\mathrm{C} \cdot\left(\mathrm{CH}_{3}\right)$ - als modifizierte Einheit im Makromolekül entstehen; das Hauptprodukt dieses Prozesse ist $\cdot \mathrm{COOCH}_{3}$. Der weitere Zerfallsprozess ähnelt der Pyrolyse von PMMA [10, 11] und ist in Abbildung 3.2 skizziert: Nach der Abspaltung der Ester-Gruppe - $\mathrm{COOCH}_{3}$ bricht das verbleibende Makromolekül an dieser Stelle unter Bildung einer Doppelbindung auf [68]. Während der Teil des Makromoleküls mit der Doppelbindung zunächst unverändert bleibt, depolymerisiert der zweite Teil unter Bildung von MMA [11]. Durch weitere Wechselwirkungen mit nachfolgenden Laserpulsen können die so entstandenen Produkte weiter zerlegt werden. Es entstehen zwei deutlich unterschiedliche Komponenten: ein Polymer mit stark reduzierter Molmasse, das sich in der Endgruppe durch eine $\mathrm{C}=\mathrm{C}$-Doppelbindung und eine fehlende Estergruppe von der Monomereinheit unterscheidet, und kleinere Bestandteile mit teilweise radikalischem Charakter, die

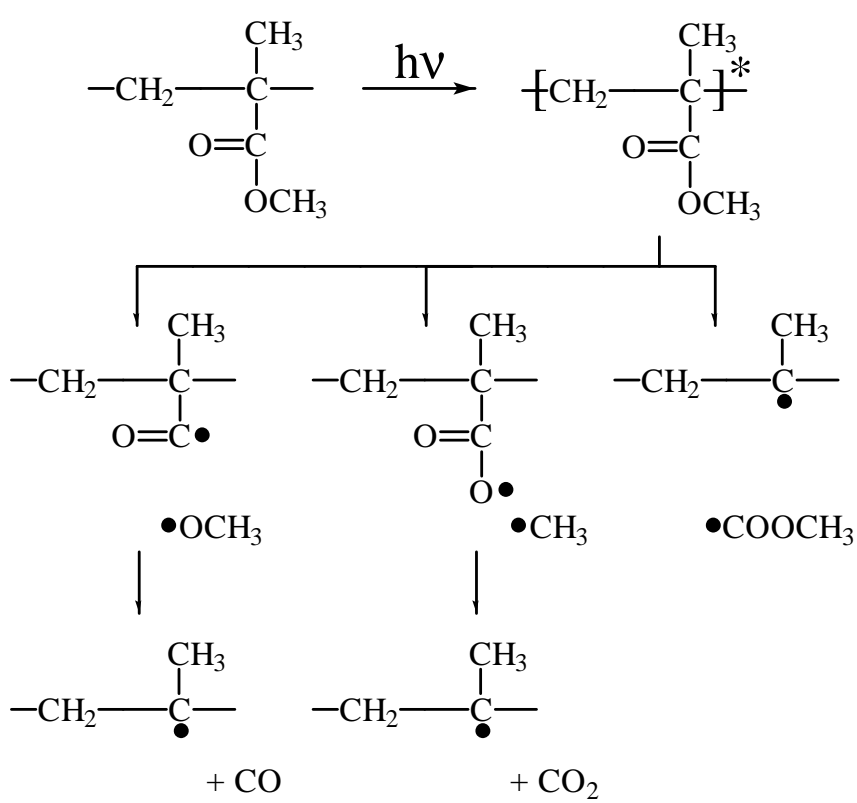

Abbildung 3.1: Schematische Darstellung der photochemischen Degradation von PMMA unter kontinuierlicher 253.7 nm-Bestrahlung nach Gupta [39]. 
(a)<smiles>CCC(C)(CC(C)(CC(C)(CC(C)[C@H](CC(=O)OC)C(C)(CC(C)(CC)C(=O)OC)C(=O)OC)C(=O)OC)C(=O)OC)C(=O)OC</smiles>

(b)<smiles>CC[C@@](C)(CC(C)(CC(C)(C)C(=O)OC)C(=O)OC)C(=O)OC</smiles><smiles>[SiH3]</smiles>

(c)<smiles>CCC(C)(C[C@@H](C)C(=O)OC)C(=O)OC</smiles><smiles>C=C(C)C(=O)OC</smiles><smiles>C=C(C)CC(C)(CC(C)(CC)C(=O)OC)C(=O)OC</smiles>

Abbildung 3.2: Schematische Darstellung der Pyrolyse von PMMA nach Manring [68]. Nach der Abspaltung der Nebengruppe (als $\cdot \mathrm{COOCH}_{3}$-Radikal, (a)) bricht die Hauptkette unter Bildung eines durch die Doppelbindung terminierten Makromoleküls ((c), rechts), und eines freien Radikals am Ende eines Makromoleküls (links). Dadurch wird eine Kettenreaktion gestartet, bei der als Produkte MMA und ein Makromolekül reduzierter Kettenlänge mit radikalischem Charakter entstehen.

im Wesentlichen durch den Zerfall der Nebengruppe - $\mathrm{COOCH}_{3}$ entstehen. 
Die Zunahme der Absorption vom UV-Bereich bis in den sichtbaren Lichtwellenbereich hinein kann durch eine Zunahme der $\mathrm{C}=\mathrm{C}$-Doppelbindungen im Material erklärt werden [61]. Auf Grund der anfänglich niedrigen Absorption mit einer Absorptionslänge von etwa $150 \mu \mathrm{m}$ [63] werden diese Veränderungen der chemischen Zusammensetzung vor allem unter der Oberfläche im Volumen realisiert. Durch eine zunehmende Absorption verkleinert sich das Volumen, in dem das Material verändert wird. Damit verbunden ist eine Zunahme der deponierten Energie pro Volumen, so dass der eigentliche Absorptionsprozess beginnt [11]. Bei einer Bestrahlung von PMMA mit UV-Pulsen höherer Energiedichte werden ähnliche Modifikationen des PMMA beobachtet; auf Grund der höheren im Material deponierten Energie kann ein nennenswerter Ablationsprozess bereits nach wenigen Pulsen beobachtet werden [85].

\subsection{Ablationsmechanismus und -produkte}

Unabhängig von photochemischem oder thermischem Charakter der Wechselwirkungen verläuft die nachfolgende Ablation für beide Mechanismen ähnlich. Die bei der Degradation entstehenden kleinen Moleküle beanspruchen ein im Vergleich zur entsprechenden Gruppe im Makromolekül höheres Volumen, MMA beispielsweise benötigt 30\% mehr Platz als die Einheit im Makromolekül [34]. Dadurch entstehen unter der Oberfläche Regionen hohen Drucks, die unter Eruption von Material aufplatzen können (volume explosion). Unterstützt wird dieser Prozess noch durch zusätzlich in das Material eingebrachte Wärmeenergie durch Photonen, die ihre Energie ohne Brechung einer Bindung als thermische Energie auf das Polymer übertragen [22]. Die Mikrostruktur einer mit 800 Pulsen $/ \mathrm{cm}^{2}$ bestrahlten PMMA-Folie (Abb. 3.3) zeigt kleine Krater mit einem Durchmesser von einigen Mikrometern und Erhebungen, die auf diese Hochdruckblasen im PMMA hindeuten. Der Mechanismus der Eruption wurde durch akustische Analysen bei Bestrahlungsexperimenten von PMMA unter ähnlichen experimentellen Bedingungen nachgewiesen [24].

Bei der Explosion werden kleine Degradationsprodukte, die die Hochdruckblase im Inneren der PMMA-Folie gebildet haben, freigesetzt. Gleichzeitig wird das Material oberhalb dieser Blase weggesprengt. Durch diesen Ablationsprozess werden zwei unterschiedliche Komponenten abgetragen: leicht modifiziertes Polymer reduzierter 


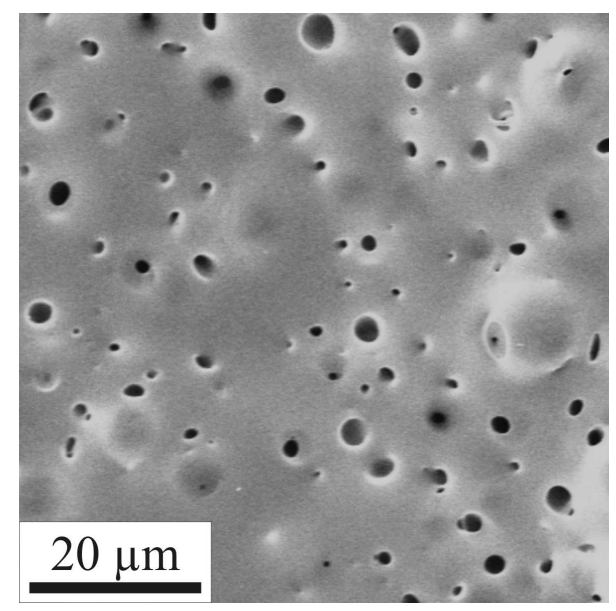

Abbildung 3.3:

Elektronenmikroskopische

Aufnahme einer mit 800 Pulsen $/ \mathrm{cm}^{2} \quad$ bestrahlten PMMA-Folie $\left(1500 \mathrm{~mJ} / \mathrm{cm}^{2}\right)$. Zur Vermeidung von Aufladungseffekten wurde die Folie vor der Untersuchung mit einer dünnen Au-Schicht überzogen.

Molmasse in den abgetragenen Partikeln neben Degradationsprodukten, die teilweise radikalischen Charakter aufweisen. Als Produkte werden durch zeitlich hochauflösende Fotografien im Wesentlichen kleine, nadelförmige Festkörper nachgewiesen, die nach einer Flugzeit von $3 \mu \mathrm{s}$ zu Tröpfchen aufschmelzen [86].

Der charakteristische Unterschied der ablatierten Materialien wird während des gesamten weiteren Depositionsprozesses beibehalten. Er äußert sich zum Beispiel anhand der unterschiedlichen Ausbreitungsgeschwindigkeiten: Die herausgesprengten Partikel weisen eine Geschwindigkeit von 150-200 m/s [84, 22] auf, $\mathrm{CO}_{2}$ als typisches Degradationsprodukt Geschwindigkeiten von 1700 m/s [22]. Die Geschwindigkeiten der Tropfen entsprechen denen der Dropletdeposition bei metallischen Systemen (150-180 m/s, [28]); große Diskrepanzen können aber bei einem Vergleich der Geschwindigkeiten kleiner Degradationsprodukte von PMMA mit Geschwindigkeiten von einigen $10^{4} \mathrm{~m} / \mathrm{s}$ bei metallischen Ionen [66] festgestellt werden. Diese Differenzen können durch unterschiedliche Ablationsprozesse dieser beiden Materialen erklärt werden: Bei der Bestrahlung von Metallen mit 248 nm-Pulsen wird bereits nach wenigen Nanosekunden Material abgetragen [27], das eine sogenannte Knudsenlage vor dem Target bildet [56]. Die hohen Geschwindigkeiten metallischer Ionen werden durch Wechselwirkungen gasförmiger Metallatome in dieser Schicht mit dem restlichen Laserpuls, der eine Länge von 20 ns hat, erzeugt. Mit einer Plasmabildung, die als starke Leuchterscheinung sichtbar wird [89], ist eine hohe Beschleunigung des Materials [29] verbunden. Diese Plasmabildung ist bei Polymeren nur sehr schwach ausgeprägt. Der Unterschied kann durch einen nennenswerten Abtrag von Material 
nach dem Ende des Laserpulses erklärt werden; bei Experimenten an Luft kann ein auflösbarer Abtrag erst nach 60 ns [84] nachgewiesen werden. Da es sich bei dem Abtragsmechanismus von PMMA um einen Volumenprozess im Inneren des Polymers ohne große Wechselwirkungen mit dem umgebenden Medium handelt, kann diese Ablationszeit in guter Näherung - im Einklang mit Untersuchungen von Kelly et al. [53] - auch auf Prozesse im Vakuum übertragen werden. Dadurch gibt es weniger Wechselwirkungen von abgetragenem Material mit dem Laserpuls. Die Plasmabildung spielt eine wesentlich geringere Rolle als bei metallischen Systemen. Die Geschwindigkeiten resultieren aus der Energie, die durch die Volumenexplosion frei wird.

Eine Analyse der abgetragenen Produkte ist erst nach dem eigentlichen Ablationsprozess möglich, der eventuelle Wechselwirkungen durch Plasmabildung beinhaltet. Auch bei diesen Analysen wird die zweikomponentige Zusammensetzung der Ablationsprodukte deutlich: Srinivasan charakterisiert die flüssigen Tröpfchen als Polymer mit einem zu PMMA identischen IR-Spektrum und einer massenmittleren Molmasse $\mathrm{M}_{W}$ von $4500 \mathrm{~g} / \mathrm{mol}\left(\mathrm{M}_{n}=2500 \mathrm{~g} / \mathrm{mol}\right)$ [82, 83]. Als zweite Komponente können kleine Moleküle mit einer Masse bis zu $100 \mathrm{~g} / \mathrm{mol}$ nachgewiesen werden $[26,82,83,59,9,10,101]$.

Diese Produkte können mit Hilfe der Massenspektroskopie charakterisiert werden. Abbildung 3.4 (a) zeigt ein bei der Ablation aufgenommenes Massenspektrum für den Molmassenbereich von 0 bis $120 \mathrm{~g} / \mathrm{mol}$; auf diesen Molmassenbereich entfallen 98,4\% des Gesamtdruckes in der Depositionskammer. Im Einzelnen können Moleküle mit Molmassen von 2, 15, 26, 28, 39, 41, 59, 69, 85 und $100 \mathrm{~g} / \mathrm{mol}$ (vgl. Tab. 3.2) nachgewiesen werden. Da durch die ionisierende Nachweismethode im Quadrupolmassenspektrometer Moleküle weiter zerlegt werden können [18], wurde zur Identifizierung der Ablationsprodukte ein Spektrum bei der thermischen Zersetzung von PMMA bei $300{ }^{\circ} \mathrm{C}$ im UHV zum Vergleich aufgenommen, dargestellt in Abbildung 3.4 (b). Obwohl bei der Pyrolyse von PMMA im Wesentlichen Monomere $(100 \mathrm{~g} / \mathrm{mol},[48,104,11])$ und in geringem Maße die Estergruppe $\left(-\mathrm{COOCH}_{3}\right.$, $59 \mathrm{~g} / \mathrm{mol}$ [68]) gebildet werden, sind in diesem Massenspektrum Moleküle mit einer Molmasse von 15, 28, 39, 41, 59, 69, 85 und $100 \mathrm{~g} / \mathrm{mol}$ nachweisbar. Diese Moleküle sind auf eine Zerlegung durch die Ionisation im Massenspektrometer zurückzuführen; mögliche von Blanchet [9] und Gupta [39] vorgeschlagene Produkte werden in Ta- 


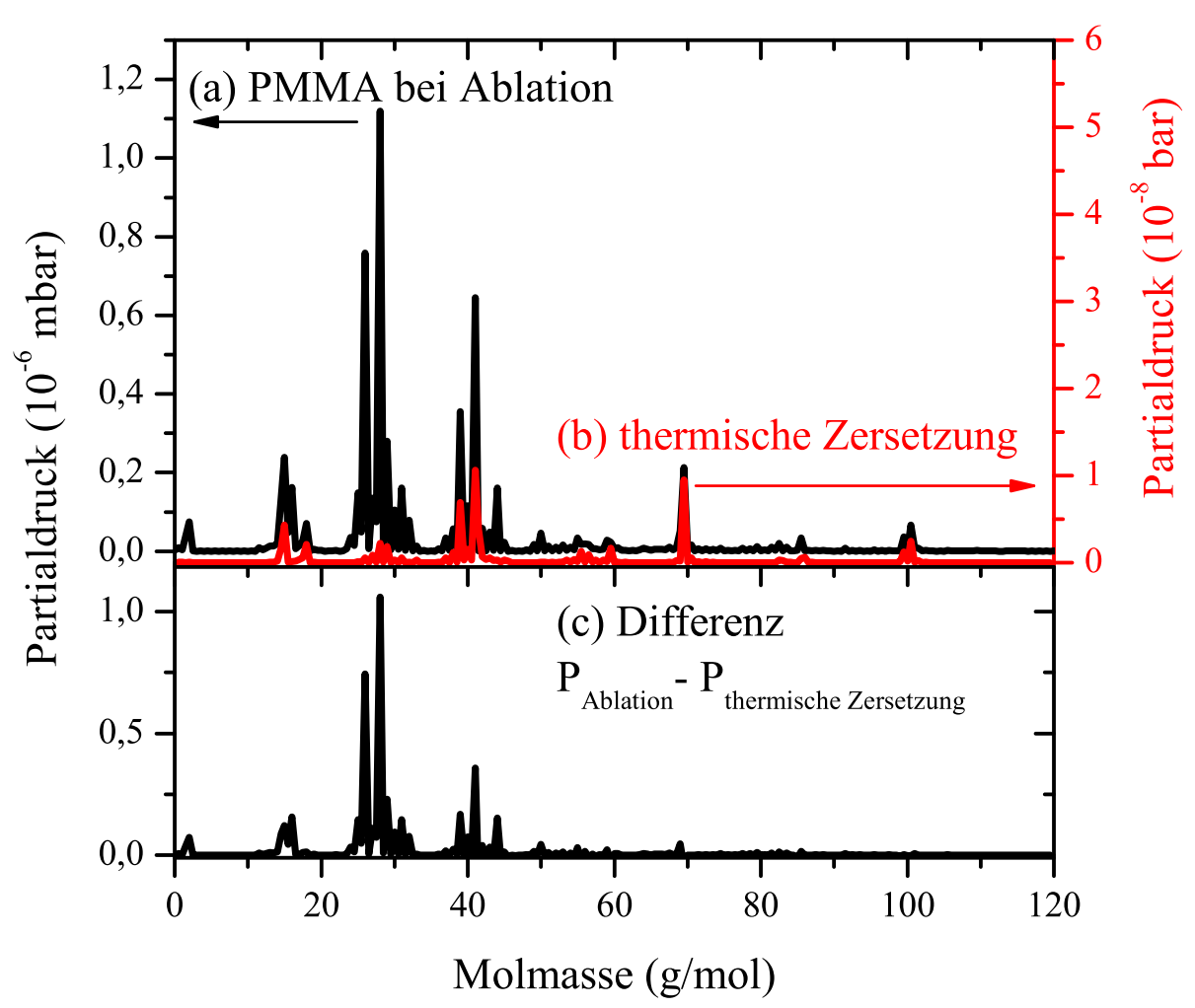

Abbildung 3.4: Massenspektrum der Ablation eines PMMA-Targets $\left(1500 \mathrm{~mJ} / \mathrm{cm}^{2}\right)$. Zum Vergleich ist ein Spektrum der Zersetzung von PMMA bei $300{ }^{\circ} \mathrm{C}$ abgebildet (b), der Übersichtlichkeit halber leicht versetzt angeordnet. Die unterschiedlichen Druckbereiche sind auf experimentelle Unterschiede zurückzuführen. Unten (c) die Differenz zwischen Ablations- und thermischem Zersetzungsspektrum, normiert auf den Partialdruck des Ablationsspektrums bei einer Molmasse von $100 \mathrm{~g} / \mathrm{mol}$ (Monomermasse).

belle 3.2 kurz zusammengefasst.

Alle Produkte, die im Massenspektrum der Pyrolyse nachgewiesen werden können, sind auch bei der Ablation zu beobachten. Abbildung 3.4 (c) zeigt die Differenz der Spektren, normiert auf den Partialdruck der Monomere mit einer Molmasse von $100 \mathrm{~g} / \mathrm{mol}$. In diesem Differenzspektrum sind die bei der thermischen Zersetzung von PMMA dominierenden Moleküle mit Molmassen von 59, 69, 85 und $100 \mathrm{~g} / \mathrm{mol}$ 


\begin{tabular}{rrrl}
\hline $\begin{array}{r}\text { Molmasse } \\
(\mathrm{g} / \mathrm{mol})\end{array}$ & Gew.-Konzentration & Zusammensetzung \\
\hline 2 & 2 & 0 & $\mathrm{c}_{a}$ \\
15 & 54 & 26 & $\mathrm{CH}_{3}$ \\
16 & 39 & 1 & $\mathrm{CH}_{4}, \mathrm{O}$ \\
18 & 19 & 15 & $\mathrm{H}_{2} \mathrm{O}$ \\
26 & 296 & 6 & $\mathrm{H}_{2} \mathrm{C}_{2}$ \\
28 & 471 & 25 & $\mathrm{CO}$ \\
29 & 122 & 21 & $\mathrm{CHO}$ \\
32 & 75 & 7 & $\mathrm{CH}_{3} \mathrm{OH}, \mathrm{O}_{2}$ \\
39 & 208 & 109 & $\mathrm{H}_{2} \mathrm{C}=\mathrm{CCH}$ \\
41 & 398 & 176 & $\mathrm{H}_{2} \mathrm{CCCH}$ \\
44 & 107 & 5 & $\mathrm{CO}_{2}$ \\
59 & 37 & 41 & $\mathrm{COOCH}_{3}$ \\
69 & 224 & 269 & $\mathrm{H}_{2} \mathrm{C}=\mathrm{C}\left(\mathrm{CH}_{3}\right) \mathrm{CO}$ \\
85 & 43 & 21 & $\mathrm{H}_{2} \mathrm{C}=\mathrm{C}\left(\mathrm{CH}_{3}\right) \mathrm{COO}$ \\
100 & 100 & 100 & $\mathrm{H}_{2} \mathrm{C}=\mathrm{C}_{\left(\mathrm{CH}_{3}\right) \mathrm{COOCH}}$ \\
\hline
\end{tabular}

Tabelle 3.2: Massenspektroskopisch nachgewiesene Produkte bei der Ablation und Pyrolyse von PMMA (vgl. Abb. 3.4), mit relativen Gewichtskonzentrationen, normiert auf $100 \mathrm{~g} / \mathrm{mol}$, und mögliche Zusammensetzungen nach Blanchet und Gupta et al. [9, 39].

weitestgehend verschwunden. Aus diesem Vergleich kann geschlossen werden, dass ein Teil der bei der Ablation nachweisbaren Moleküle den bei der thermischen Degradation von PMMA entstehenden Monomeren entspricht. Durch eine Integration der Spektren lässt sich der Anteil dieser Monomere auf etwa 40 Gew.- \% der gesamten gasförmigen Ablationsprodukte abschätzen.

Zusätzlich entstehen bei der Ablation noch weitere Produkte, die im Differenzspektrum als Maxima bei Molmassen von 2, 14, 16, 26, 28, 39, 41, 44 und $69 \mathrm{~g} / \mathrm{mol}$ sichtbar sind. Diese Moleküle entsprechen den Produkten, die bei einer photochemischen Degradation von PMMA durch die Teilzerlegung der Estergruppe gebildet werden (vgl. Tab. 3.2 und Abb. 3.1, [39]). Auf Grund der Ionisation kann eine Zerlegung der Estergruppe im Massenspektrometer nicht ausgeschlossen werden; die Experimente weisen aber klar auf eine Modifikation von Nebengruppen durch 
Wechselwirkungen zwischen UV-Laserpulsen und PMMA hin.

Der wesentliche Schlüssel zum Verständnis des Ablationsprozesses ist somit die Wechselwirkung zwischen Laserstrahlung und Polymer. Ihre Bedeutung wird in der Absorptionsabhängigkeit des Ablationsmechanismus ersichtlich: Bei der Ablation von PMMA mit einer Wellenlänge von $248 \mathrm{~nm}\left(\alpha=4 \cdot 10^{2} \mathrm{~cm}^{-1}[93]\right)$ teilen sich die Produkte in zwei Komponenten auf: ein leicht modifiziertes Polymer mit einer reduzierten Molmasse, das in Tröpfchenform vom Target zum Substrat transferiert wird und gasförmige Degradationsprodukte von PMMA, die zu etwa 40 Gew.- $\%$ als Monomer identifiziert werden konnten. Die übrigen Moleküle lassen sich durch eine Zerlegung der Estergruppe erklären. Die Ablationsprodukte bei einer Bestrahlung von PMMA mit Laserpulsen einer Wellenlänge von 193 nm [82, 83], wo PMMA einen Absorptionskoeffizienten von 4,4 $10^{3} \mathrm{~cm}^{-1}$ [93] aufweist, unterscheiden sich deutlich von diesen Produkten; bei einer weiteren Reduzierung der Wechselwirkung ( $\alpha \simeq 1 \cdot 10^{2} \mathrm{~cm}^{-1}$ bei $308 \mathrm{~nm}$ [93]) ändert sich die Verteilung der Produkte erneut [64]. Diese Absorptionsabhängigkeit der Ablation ist verantwortlich für unterschiedliche Mechanismen, die bei der Bestrahlung unterschiedlicher Polymere diskutiert werden: Während für stark UV-Strahlung-absorbierende Polymere wie Polycarbonat (PC) oder Polyimid (PI) $\left(2,8 \cdot 10^{5} \mathrm{~cm}^{-1}\right.$ bei $248 \mathrm{~nm}$, [79]) bereits bei moderaten Energiedichte eine komplette Zerlegung in kleine Moleküle [87] und Deposition von Schichten mit deutlich geänderter Zusammensetzungen und Eigenschaften [98] diskutiert wird, kann für andere schwach absorbierende Polymere wie Poly(tetrafluorethylen) (PTFE) ein Abtrag von festem Polymer [7, 8, 44] nachgewiesen werden, der vergleichbar zur Ablation von PMMA ist. 


\section{4 \\ Deposition auf dem Substrat}

Nach der Ablation werden beide Komponenten - Polymerdroplets und kleine Degradationsprodukte - zum Substrat übertragen. In diesem Kapitel wird die Deposition beider Komponenten beschrieben. Besonderes Augenmerk liegt dabei auf den Eigenschaften der Filme, die die zweikomponentigen Ablationsprodukte von der Mikrostruktur über die chemische Zusammensetzung der Filme bis hin zu makroskopisch-mechanischen Eigenschaften repräsentieren.

\subsection{Mikrostruktur laserdeponierter Filme: Dropletdeposition und vernetzte Schicht}

Abbildung 4.1 (a) zeigt eine elektronenmikroskopische Aufnahme eines PMMAFilmes nach der Deposition von 200 Pulsen PMMA. Die Mikrostruktur der Filme ist durch die zweikomponentige Zusammensetzung der Ablationsprodukte geprägt: Sie wird wesentlich durch die Deposition von Tröpfchen bestimmt, die als zylinderförmige Inseln auf dem Substrat erscheinen. Kleinere Degradationsprodukte bilden einen kontrastarmen Film zwischen den Droplets. Elektronenmikroskopische Aufnahmen lassen runde Inseln mit einem Durchmesser von 0,3 bis $8 \mu \mathrm{m}$ erkennen. Die Größenverteilung zeigt viele kleine mit einem Durchmesser von weniger als 1,5 $\mu \mathrm{m}$ und nur wenige große Inseln auf. Diese Inseln weisen teilweise eine sehr flache, zylinderförmige Struktur, teils eine halbkugelartige Form auf. Diese charakteristische Form und Höhe der Inseln wird von der Temperatur und der Abkühlgeschwindigkeit der de- 


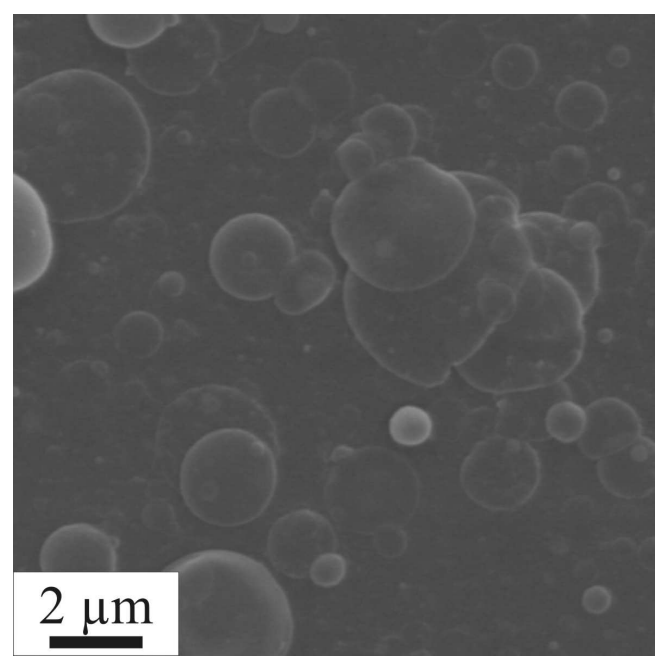

(a) PLD-PMMA

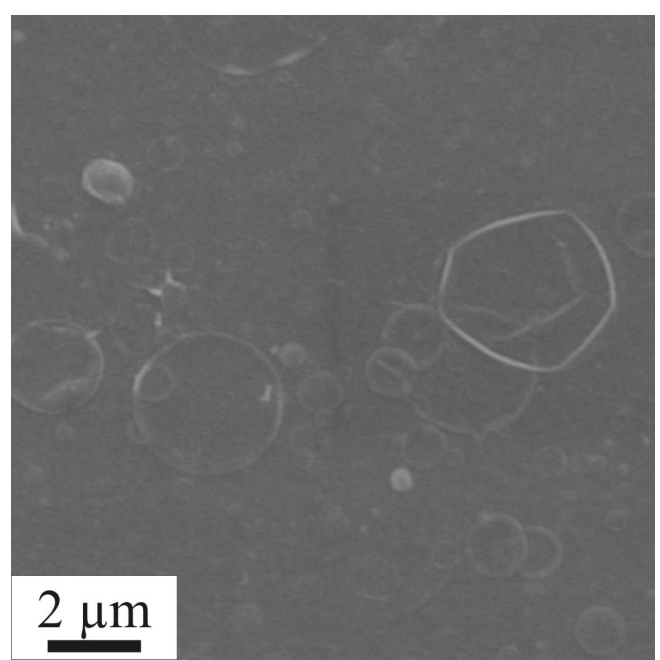

(b) Rückstand

Abbildung 4.1: Rasterelektronenmikroskopische Aufnahme eines PMMAFilmes nach der Herstellung (200 Pulse, $1500 \mathrm{~mJ} / \mathrm{cm}^{2}$ ) (a) und Rückstand nach der Behandlung in THF (b).

ponierten Tropfen bestimmt: Bei schneller Abkühlrate oder geringer Temperatur des Tropfens beim Auftreffen auf das Substrat entstehen dabei eher kugelförmige, bei langsamem Abkühlen flache, zylinderförmige Inseln. Experimentell wurde die Deposition von Tropfen (Droplets) auch bei Metallen [55, 27] oder Keramiken [35] beobachtet. Die Volumenexplosion beim PMMA unterscheidet sich aber klar vom Bildungsmechanismus metallischer Tröpfchen, wo vielmehr die Aufrauung der Targets für die Dropletbildung [51, 52] verantwortlich ist.

Diese Tröpfchen entsprechen einem nur leicht modifizierten Polymer reduzierter Molmasse. Sie sind in organischen Lösungsmitteln wie Aceton oder Tetrahydrofuran (THF) löslich. Abbildung 4.1 belegt dies anhand eines Vergleichs von elektronenmikroskopischen Aufnahmen eines laserdeponierten PMMA-Filmes im hergestellten Zustand mit dem gleichen Film nach der Behandlung mit THF. Nach dem Lösen des Polymers sind nurmehr wenige unveränderte Inseln zu erkennen. Daneben werden ringförmige Strukturen sichtbar, die wie geplatzte Blasen wirken, und durch das Herauslösen von PMMA aus dem Inneren der Droplets entstanden sind. Bei diesem Lösungsprozess bleibt eine vernetzte Haut, die die Oberfläche der Inseln gebildet hat, 
übrig. Diese Haut entsteht beim Überwachsen der Inseln durch eine nachfolgende Deposition von reaktivem Material.

Detaillierter lassen sich die Dropletinseln mit Hilfe eines Rasterkraftmikroskops untersuchen. Abbildung 4.2 zeigt einen Ausschnitt einer Probe als z-Kontrastbild - in Zusammenarbeit mit dem I. Physikalischen Institut der Universität Göttingen entstanden - mit einer deutlichen Höhenüberzeichnung, bzw. als zweidimensionale Aufnahme in Abbildung 4.3 (a). Die hier sichtbaren Droplets weisen Höhen von 50 bis $600 \mathrm{~nm}$ auf. Die Höhenverteilung ist bimodal: So können kleine Tröpfchen mit einem Durchmesser von weniger als $2 \mu \mathrm{m}$ und einer Höhe von bis zu $600 \mathrm{~nm}$ nachgewiesen werden, wohingegen Inseln mit einer lateralen Ausdehnung von mehr als $2 \mu \mathrm{m}$ eine maximale Höhe von $250 \mathrm{~nm}$ zeigen. Als Beispiel ist ein Höhenprofil einer solchen Insel mit einem Durchmesser von 5,6 $\mu \mathrm{m}$ und einer Höhe von $212 \mathrm{~nm}$ in Abbildung 4.3 (b) dargestellt. Die mittlere Inselhöhe beträgt etwa $170 \mathrm{~nm}$.

Nach der Behandlung mit THF nimmt die Inselanzahl und mittlere Höhe der

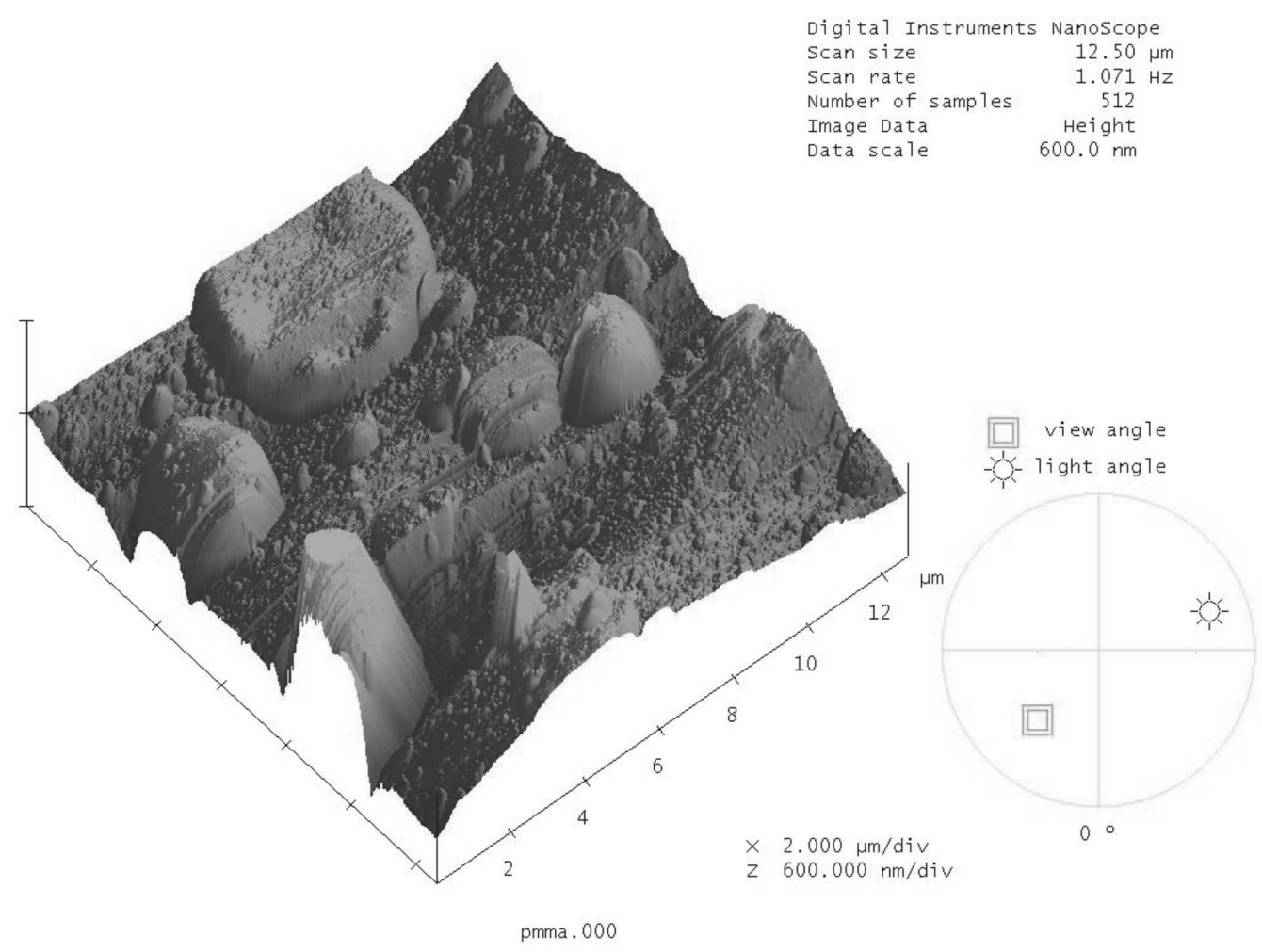

Abbildung 4.2: AFM-z-Kontrast-Abbildung eines PMMA-Filmes (200 Pulse, $1500 \mathrm{~mJ} / \mathrm{cm}^{2}$ ) mit 3,3-facher Höhenüberzeichnung. 


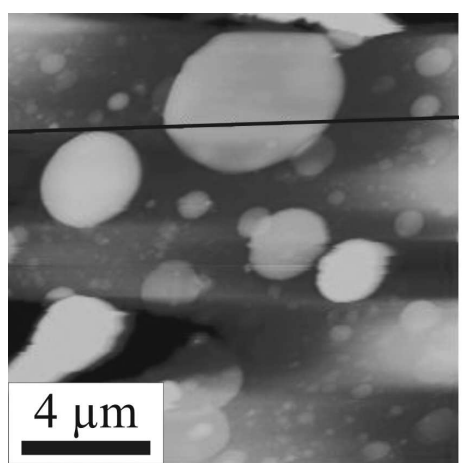

(a) Übersicht

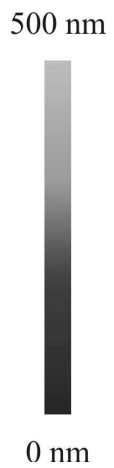

$0 \mathrm{~nm}$

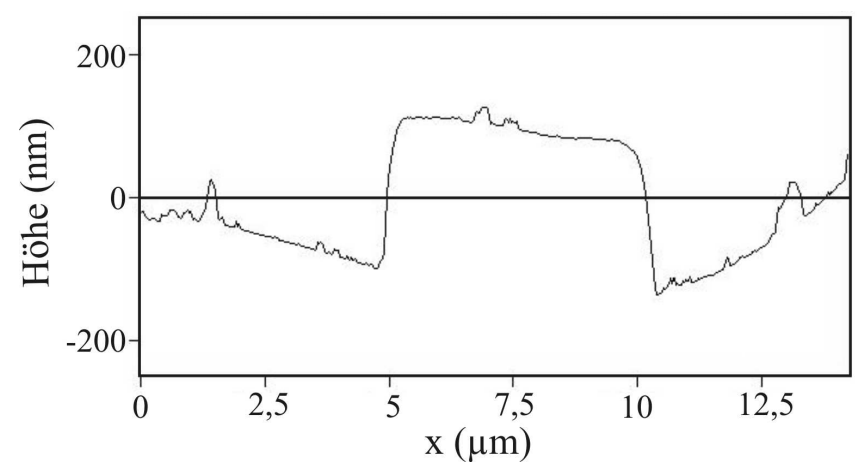

(b) Höhenprofil

Abbildung 4.3: AFM-z-Kontrast einer laserdeponierten PMMA-Probe (200 Pulse, $1500 \mathrm{~mJ} / \mathrm{cm}^{2}$ ) und zugehöriges Höhenprofil eines Droplets mit einer Höhe von $212 \mathrm{~nm}$ und einer lateralen Ausdehnung von 5,6 $\mu \mathrm{m}$.

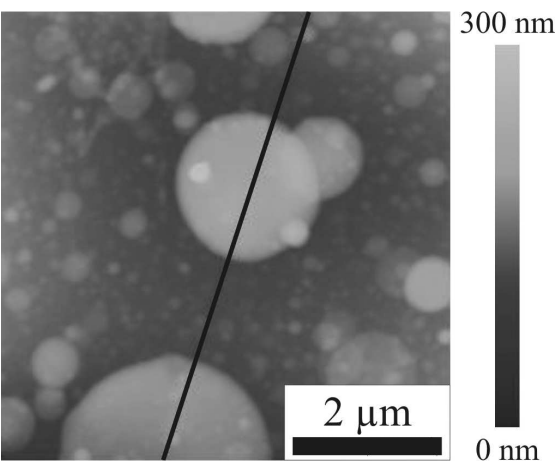

(a) Übersicht

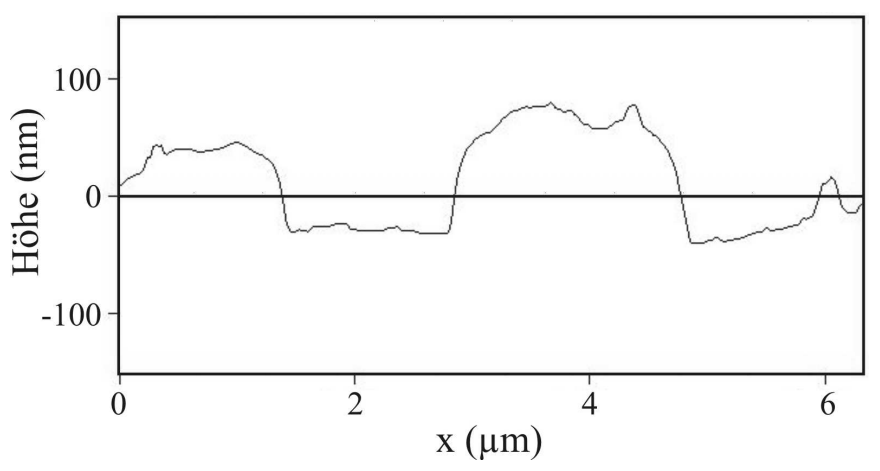

(b) Höhenprofil

Abbildung 4.4: AFM-z-Kontrast eines PMMA-Films (200 Pulse, $1500 \mathrm{~mJ} / \mathrm{cm}^{2}$ ) nach der Behandlung in THF (vgl. Abb. 4.1) und $\mathrm{zu}-$ gehöriges Höhenprofil durch ein Droplet mit einer Höhe von $110 \mathrm{~nm}$ und einer lateralen Ausdehnung von $2 \mu \mathrm{m}$.

Inseln auf $70 \mathrm{~nm}$ ab. Abbildung 4.4 zeigt entsprechende rasterkraftmikroskopische Aufnahmen mit zugehörigem Höhenprofil nach der Behandlung mit THF.

Neben den Droplets werden kleinere Degradationsprodukte ablatiert. Diese bilden bei der Deposition einen glatten Film zwischen den Droplets (vgl. Abb. 4.1). Abbildung 4.5 zeigt eine Röntgenreflektometriemessung der gleichen Probe, die in 


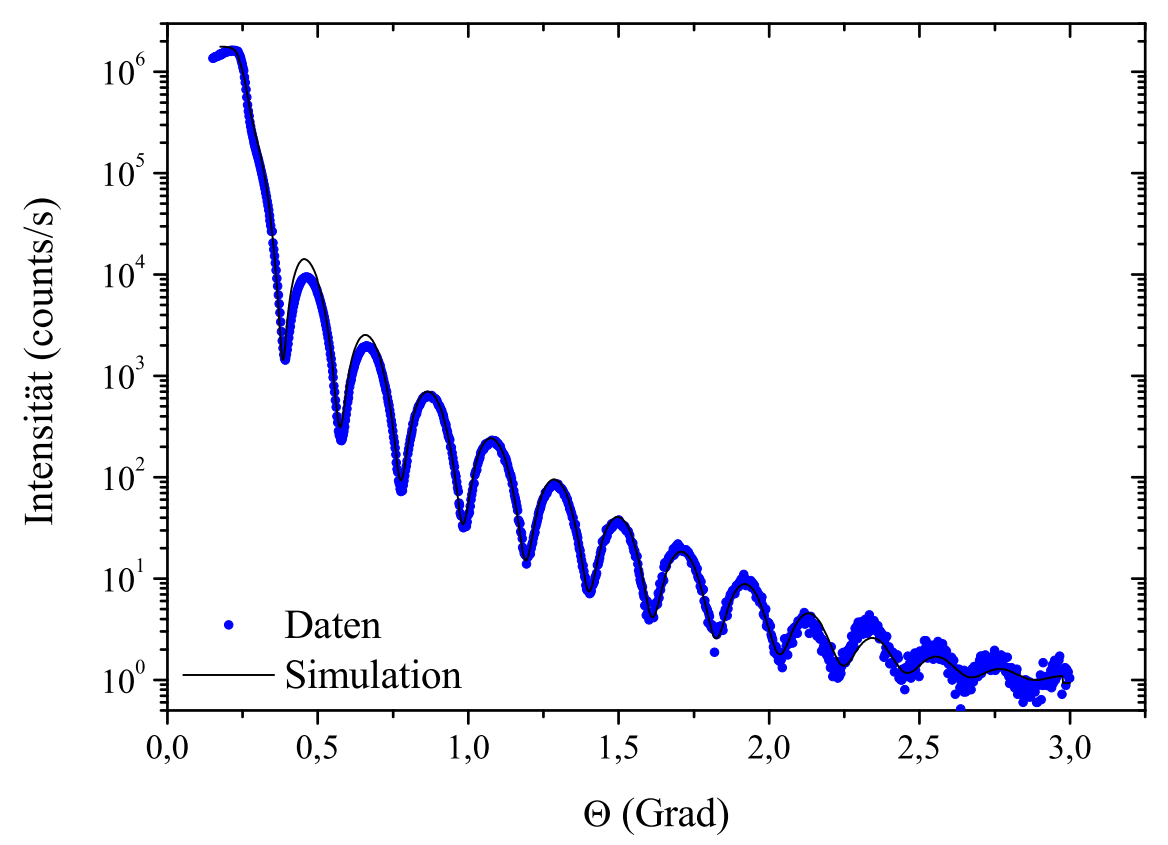

Abbildung 4.5: Reflektometrie eines PMMA-Films (200 Pulse, $1500 \mathrm{~mJ} / \mathrm{cm}^{2}$ ). Zum Vergleich ist auch die IMD-Simulation der Schicht mit aufgetragen; der Film hat eine Dicke von $24,1 \mathrm{~nm}$, bei einer Dichte von $1,4 \mathrm{~g} / \mathrm{cm}^{3}$ und einer Rauigkeit von $0,5 \mathrm{~nm}$; die Substratrauigkeit wurde mit $0,6 \mathrm{~nm}$ modeliert.

den Aufnahmen 4.1 - 4.4 mit Hilfe der Rasterelektronen- und Rasterkraftmikroskopie charakterisiert wurde. Deutlich sind die für Einfachschichten typischen KiessigFringes [36] zu sehen, die auf eine homogene Schicht hindeuten. Genauere Simulationen mit Hilfe des IMD-Softwarepakets [103] zeigen eine Schichtdicke von 24,1 nm bei einer Dichte des Polymers von $1,4 \mathrm{~g} / \mathrm{cm}^{3}$ und einer Oberflächenrauigkeit $\sigma$ von nur 0,5 nm. Ein Beitrag der Inseln kann durch die unterschiedlichen Höhen der Droplets vernachlässigt werden; gleichzeitig liegt die Eindringtiefe von Röntgenstrahlung mit einer Wellenlänge von 0,17902 nm in PMMA bei $855 \mu \mathrm{m}$ [45], so dass die Tröpfchen glatt durchstrahlt werden können, und Information auch aus der Schicht unter den Inseln gewonnen werden kann.

Diese Schicht erfährt durch eine Behandlung mit organischen Lösungsmitteln keine signifikanten Veränderungen, wie in Abbildung 4.6 beim Vergleich der Re- 


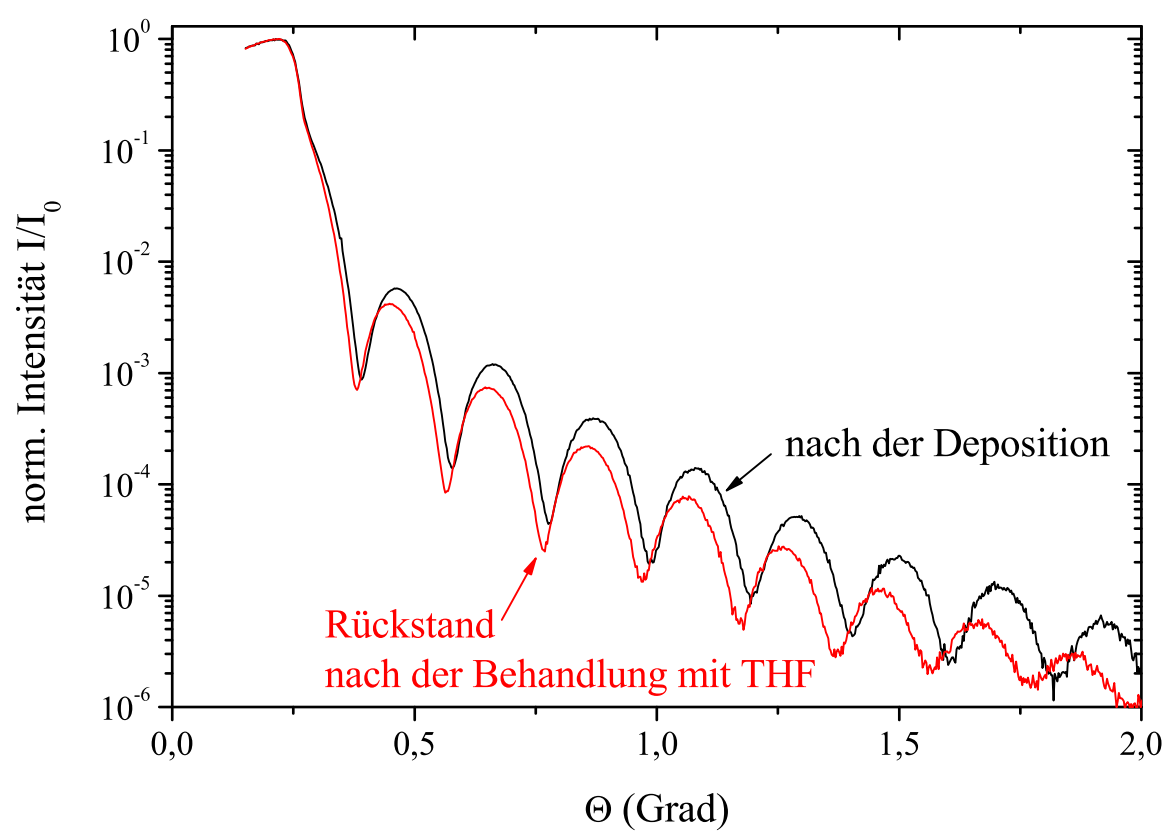

Abbildung 4.6: Reflektometrie eines PMMA-Films (200 Pulse, 1500 mJ/cm²), dessen lösliche Bestandteile in THF gelöst wurden. Das Lösungsmittel ist durch Lagerung des Filmes im UHV $\left(p<10^{-7}\right.$ mbar) für 48 Stunden entfernt worden. Zum Vergleich ist auch die Reflektometrie der unbehandelten Probe dargestellt.

flektogramme der unbehandelten und in THF gelösten Schicht zu erkennen ist. Simulationen der Schicht ergeben eine leichte Zunahme der Schichtdicke der in THF gelösten Probe von 24,1 nm auf 24,4 nm bei einer Dichte von 1,3 g/ $\mathrm{cm}^{3}$ und einer Rauigkeit von 1,1 nm. In Tabelle 4.1 sind die Daten der IMD-Simulationen des

\begin{tabular}{lcccc}
\hline & $d$ & $\sigma$ & $\rho$ & $d \cdot \rho$ \\
& $(\mathrm{nm})$ & $(\mathrm{nm})$ & $\left(\mathrm{g} / \mathrm{cm}^{3}\right)$ & \begin{tabular}{c}
$\left.d \mathrm{~g} / \mathrm{cm}^{2}\right)$ \\
\hline unbehandelter Film
\end{tabular} \\
24,1 & 0,5 & 1,4 & $3,30 \cdot 10^{-10}$ \\
Rückstand & 24,4 & 1,1 & 1,3 & $3,05 \cdot 10^{-10}$ \\
\hline
\end{tabular}

Tabelle 4.1: Vergleich IMD-Simulationsdaten für den unbehandelten Film (200 Pulse, $1500 \mathrm{~mJ} / \mathrm{cm}^{2}$ ) und den in THF unlöslichen Rückstand. 
Filmes vor und nach der Behandlung mit THF zusammengefasst. Diese Resistenz gegen organische Lösungsmittel deutet auf eine vernetzte Struktur der Schicht hin, die durch chemische Reaktionen kleiner Degradationsprodukte bei der Deposition auf dem Substrat gebildet wird.

Eine Zunahme der Schichtdicke kann auf ein Quellen des Filmes infolge der Behandlung mit Lösungsmitteln zurückgeführt werden [37]. Während der Behandlung mit Lösungsmitteln wird die Dichte der vernetzten Schicht leicht reduziert. Eine Abschätzung des analysierten Filmgewichtes pro Fläche $d \cdot \rho$, wie sie in Tabelle 4.1 enthalten ist, zeigt, dass das analysierte Filmgewicht der mit THF behandelten Probe um $8 \%$ verringert ist, was auf einen geringfügigen Massenverlust hindeutet; auch die Zunahme der Rauigkeit weist auf kleine Veränderungen in der vernetzten Schicht hin.

Die Charakterisierung der Mikrostruktur mit Rasterkraftmikroskopie und Röntgenreflektometrie bietet die Möglichkeit, die Volumenanteile des in den Tröpfchen deponierten Polymers und des vernetzten dünnen Filmes abzuschätzen. Grundlage dieser Abschätzung ist die Form der Inseln, deren laterale Ausdehnung i. A. sehr viel größer ist als die Höhe. In einer einfachen Beschreibung lassen sich die Inseln daher als Zylinder ansehen. Mit Hilfe der Auswertesoftware NanoScope R III, Version 5.12r3, wurde die Fläche und Höhe der deponierten Droplets (mit einer unteren Schwelle von $30 \mathrm{~nm}$ ) und damit das Volumen bestimmt. Unter der Annahme rein zylinderförmiger Inseln lässt sich eine mittlere Schichtdicke von etwa $56 \mathrm{~nm}$ angeben. Diese Abschätzung ist geometrisch als eine obere Grenze des deponierten Volumens zu betrachten; eine analoge Abschätzung als untere Grenze, die die Droplets als Ellipsoide beschreibt, ergibt eine mittlere Schichtdicke von $36 \mathrm{~nm}$. Auf Grund der Form großer Inseln erscheint der erste Ansatz jedoch realistischer. Beide Abschätzungen können allerdings Rauigkeiten und Inhomogenitäten der Schicht nicht berücksichtigen und sollen daher auch nur als grobe Annäherungen an das deponierte Volumen verstanden werden. Die so bestimmte mittlere Schichtdicke entspricht der Annahme, dass alles deponierte Material als homogene Schicht aufwächst. Es sei hier darauf hingewiesen, dass eine solche, dicke, homogene Schicht aus den Aufnahmen der Rasterkraft- und Rasterelektronenmikroskopie eindeutig ausgeschlossen werden kann.

Mit Hilfe dieser Abschätzung lassen sich aber die deponierten Volumenbruch- 
teile der homogenen dünnen Schicht und der deponierten Droplets vergleichen. Die insgesamt deponierte mittlere Schichtdicke, die sich aus einer dünnen, homogenen Schicht auf dem Substrat, und den deponierten Inseln zusammensetzt, lässt sich auf eine Dicke von 60-80 nm abschätzen. Damit entspricht das in den Inseln deponierte Volumen zwischen 60 und $70 \%$ des Schichtvolumens. Die Aufwachsrate von 0,3 bis 0,4 nm/Puls entspricht etwa der Rate von PMMA, die man bei einer Interpolation auf eine Energiedichte von $1500 \mathrm{~mJ} / \mathrm{cm}^{2}$ aus Rutherford-BackscatteringExperimenten (RBS) an Ag/PMMA/Ag-Schichtsystemen [91] bestimmen kann. Sie ist abhängig von Depositionsparametern wie Energiedichte und Target-SubstratAbstand [91]. Experimentell ist ein in organischen Lösungsmitteln löslicher Anteil von 70-80 Gew.\% aus gravimetrischen Analysen direkt zugänglich. Die Vergleichbarkeit des Dropletanteils mit dem löslichen Filmanteil bestätigt die Deposition von chemisch kaum modifiziertem Polymer.

Im Vergleich zu anderen dropletbildenden Systemen wie Metallen [55, 27], wo ein Dropletanteil von wenigen Prozent beobachtet wird, ist ihr Anteil an der Gesamtschicht relativ hoch. Aus der Diskussion der Metall-Dropletbildung können auch Vorschläge abgeleitet werden, wie diese vermieden werden können. Auf Grund der unterschiedlichen Ablationsmechanismen erscheint der erfolgsversprechende Ansatz eine Trennung der Komponenten durch ein mechanisches Filter zu sein, das wegen der Geschwindigkeitsunterschiede Droplets und kleine Degradationsprodukte vor dem Auftreffen auf dem Substrat trennt [65]. Insbesondere zur Herstellung von glatten Schichten, wie sie vergleichbar mit Hilfe des Spincoating-Verfahrens aufgebracht werden können, kann ein solches Filter von Interesse sein. Da die Eigenschaften der Filme aber durch die Koexistenz von Droplets und vernetzter Schicht - wie in den folgenden Kapiteln diskutiert - bestimmt werden, würden die Eigenschaften der Filme durch eine Trennung beider Komponenten stark modifiziert werden.

\subsection{Molmassenverteilung deponierter PMMA-Droplets}

Abbildung 4.7 zeigt ein Größen-Ausschluss-Chromatogramm (Size exclusion, SE) einer laserdeponierten Probe, das in Zusammenarbeit mit dem Institut für physi- 


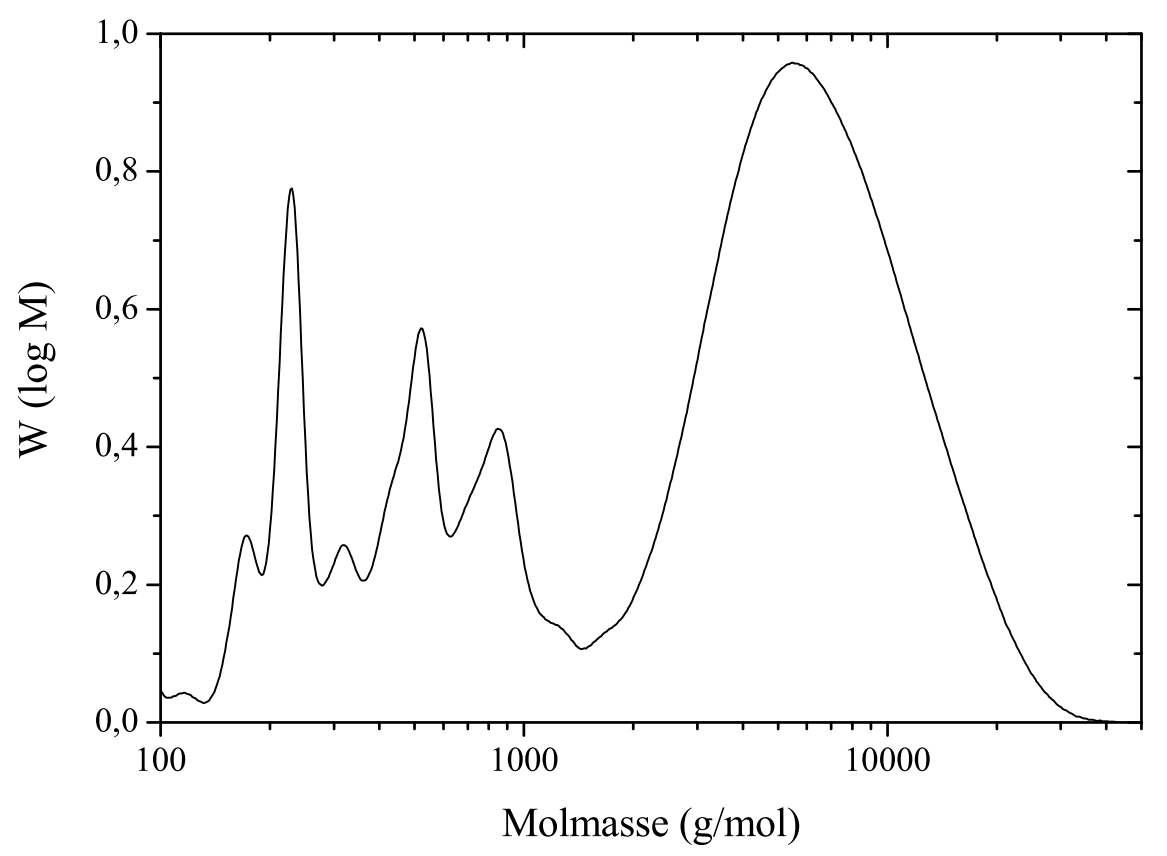

Abbildung 4.7: Molmassenverteilung des löslichen Anteils eines laserdeponierten PMMA-Filmes (1500 mJ/ $\mathrm{cm}^{2}, 10000$ Pulse).

kalische Chemie der Universität Göttingen aufgenommen wurde. Auf Grund der Deposition von Droplets entsprechen zwischen 60 und 80\% des Materials einem unvernetzten Polymer, das sich durch eine reduzierte Molmasse auszeichnet. Entsprechend weist das Chromatogramm ein breites Maximum mit einer massenmittleren Molmasse $\mathrm{M}_{W}$ von etwa $8 \cdot 10^{3} \mathrm{~g} / \mathrm{mol}\left(\mathrm{M}_{n}=5,3 \cdot 10^{3} \mathrm{~g} / \mathrm{mol}\right)$ auf, das den Droplets entspricht. Dieses Ergebnis ist im Wesentlichen unabhängig von der Molmasse des verwendeten Targets; erst bei Ausgangsmaterialien mit einer Molmasse von weniger als $8 \mathrm{~kg} / \mathrm{mol}$ verringert sich auch die Molmasse der Droplets. Abbildung 4.8 zeigt dies exemplarisch durch einen Vergleich eines SE-Chromatogramms einer laserdeponierten Probe mit dem entsprechenden, durch radikalische Polymerisation mit dem Institut für Physikalische Chemie hergestellten Targetmaterial $\left(\mathrm{M}_{W}=3,2 \mathrm{~kg} / \mathrm{mol}\right)$. Der hochmolekulare Anteil der laserdeponierten Probe entspricht dem des Ausgangsmaterials, was als indirekter Beweis eines Übertrages von kompletten Makromolekülen mit einer Molmasse von bis zu 8 kg/mol verstanden werden kann. Srinivasan 


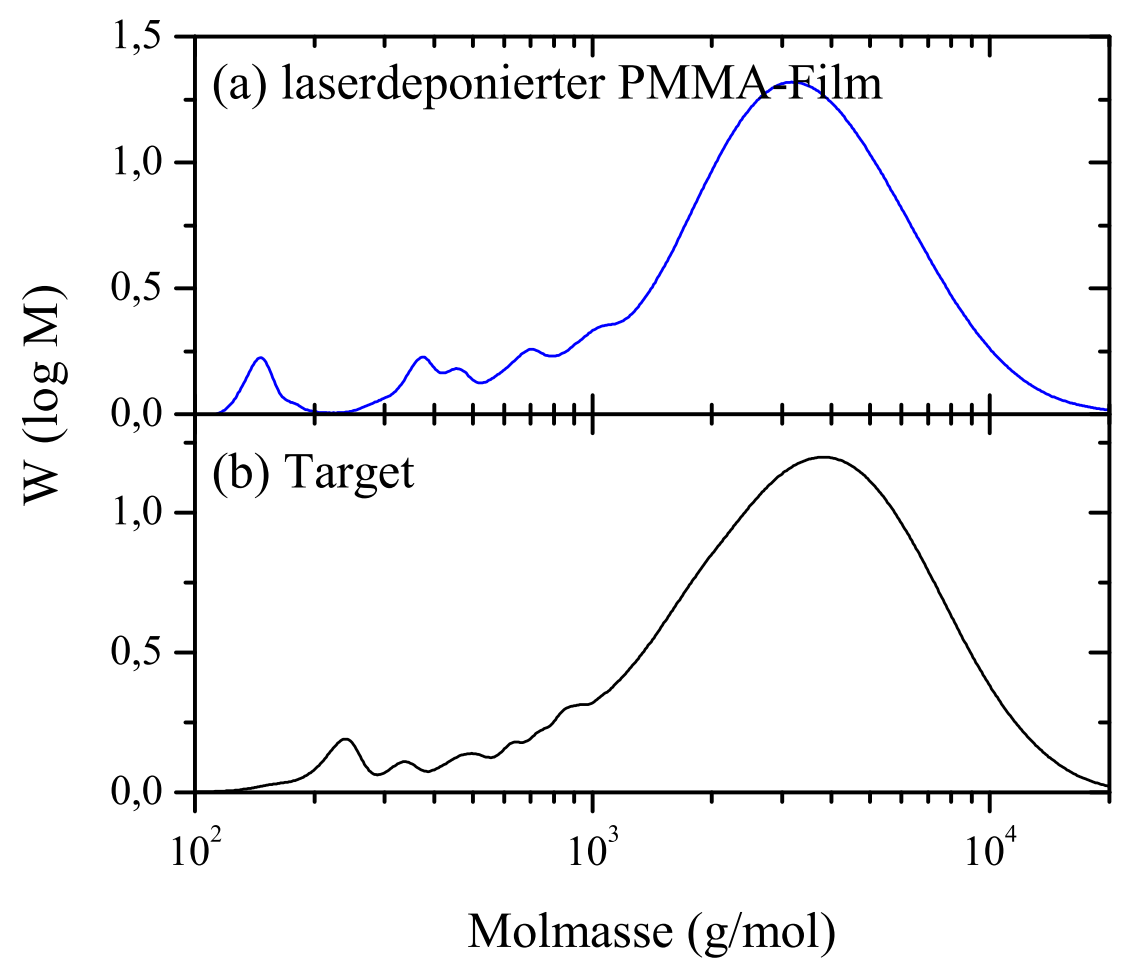

Abbildung 4.8: SE-Chromatogramm eines mittels radikalischer Polymerisation hergestellten Targets $\left(\mathrm{M}_{W} \simeq 3,2 \mathrm{~kg} / \mathrm{mol}\right)$ (b) und Chromatogramm eines entsprechenden laserdeponierten Filmes (a).

und Blanchet beschreiben laserdeponierte Filme mit Molmassen zwischen 4,5 und 10 kg/mol [82, 83, 9]; Diskrepanzen zu den hier geschilderten Werten können durch unterschiedliche experimentelle Parameter (z.B. Laserwellenlänge, Absorption von PMMA, vgl. Kap. 3) erklärt werden.

Neben diesem Hauptmaximum, dessen Anteil am löslichen Film sich durch eine Integration auf über 80 Gew.-\% abschätzen lässt, können auch kleinere Moleküle bei 114, 171, 228, 318, 522 und $843 \mathrm{~g} / \mathrm{mol}$ beobachtet werden. Diese Maxima deuten auf die Deposition von Oligomeren hin. Ihre Absolutposition ist auf Grund einer fehlenden Kalibrierung der SEC-Säulen für Massen unterhalb von $800 \mathrm{~g} / \mathrm{mol}$ fehlerbehaftet. Eine eventuelle Periodizität dieser Bestandteile lässt sich anhand der Wendepunkte des SE-Chromatogramms untersuchen. Abbildung 4.9 (a) zeigt die 


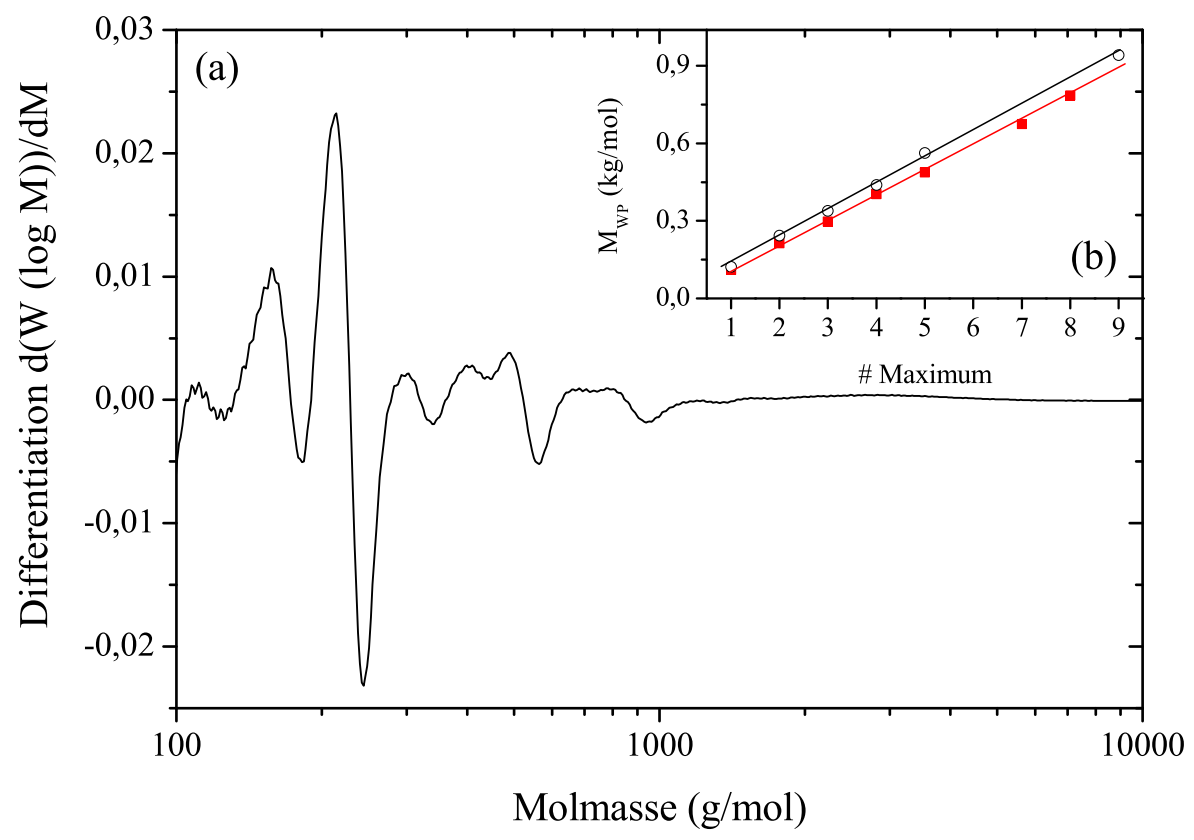

Abbildung 4.9: Ableitung (a) und daraus bestimmte Wendepunkte $\mathrm{M}_{\mathrm{WP}}$ (b) eines SE-Chromatogramms eines PMMA-Filmes $\left(1500 \mathrm{~mJ} / \mathrm{cm}^{2}, 10000\right.$ Pulse, vgl. Abb. 4.7). Der äquidistante Abstand der Wendepunkte deutet auf Dimere und Oligomere im THF-löslichen Anteil hin.

Differentiation des Chromatogramms (vgl. Abb. 4.7). Die daraus bestimmten Wendepunkte, die in Abbildung 4.9 (b) dargestellt sind, steigen linear mit den Maxima an und weisen einen gleichbleibenden Abstand zwischen rechts- und linksseitigen Wendepunkten um ein Maximum herum auf. Dieser Zusammenhang deutet eine Periodizität der Moleküle und damit eine Deposition von Dimeren und kleinen Oligomeren an. Da die Massenspektren der Ablation keine Massen oberhalb der Monomermasse aufweisen (vgl. Abb. 3.4 oder [26, 82, 83, 59, 9]), deutet dieser Befund auf eine chemische Reaktion ähnlich einer Polymerisation von Monomeren zu Oligomeren hin. Sowohl Massenspektren als auch Rauigkeitsänderungen der vernetzten Schicht legen den Schluss nahe, dass die Oligomere teilweise auch aus dem vernetzten Anteil des deponierten Filmes gelöst werden. 


\subsection{Struktur und Zusammensetzung von PMMA-Filmen}

Etwa $70 \%$ des Filmmaterials bestehen aus einem unvernetzten und nur leicht modifizierten Polymer. Entsprechend weisen laserdeponierte PMMA-Filme eine ähnliche Struktur und Zusammensetzung wie das Ausgangsmaterial auf. Sie wachsen amorph [16] auf, wie an der Röntgenbeugung in Abbildung 4.10 sichtbar wird: Das Diffraktogramm des laserdeponierten Filmes zeigt ein charakteristisches amorphes Maximum

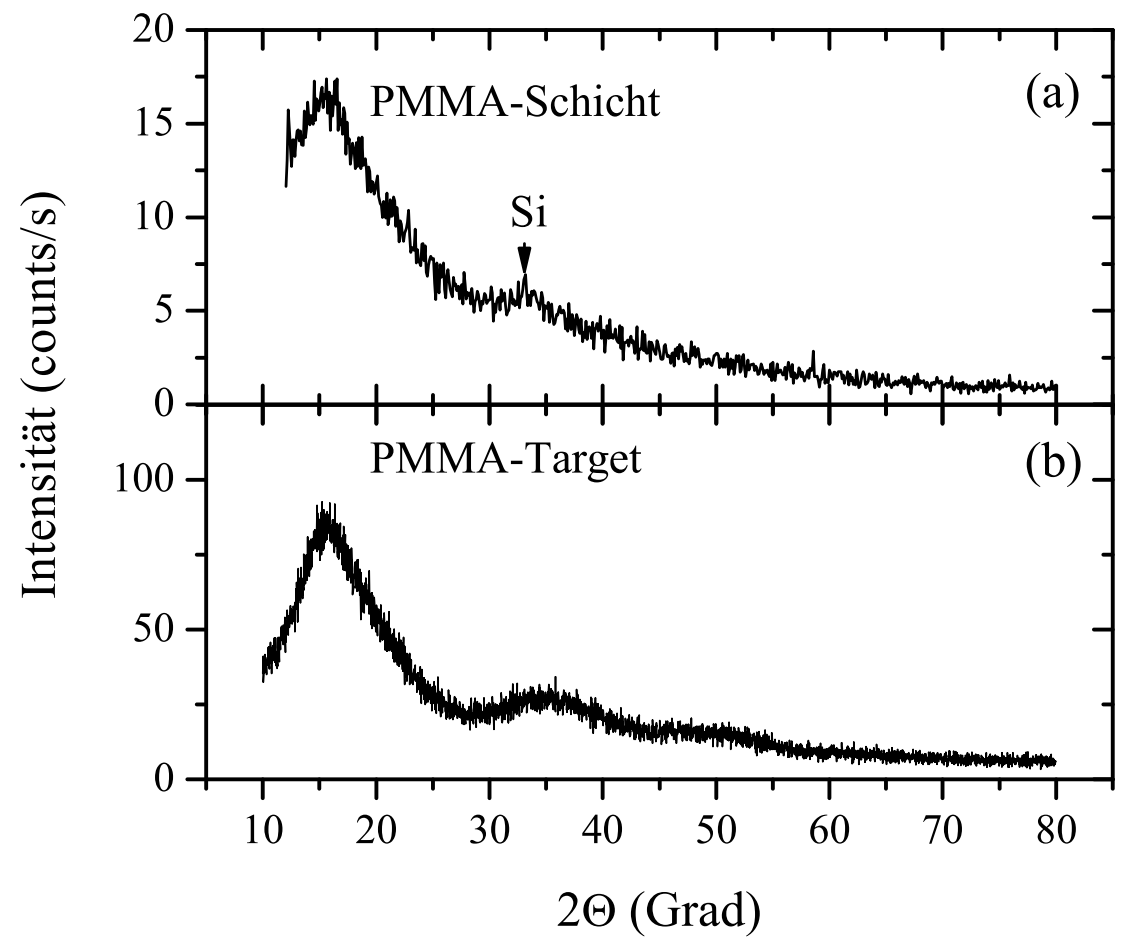

Abbildung 4.10: Diffraktogramm einer laserdeponierten PMMA-Schicht (50000 Pulse, $1500 \mathrm{~mJ} / \mathrm{cm}^{2}$ ) (a) und einer PMMA-Folie (b). Im Diffraktogramm des laserdeponierten Filmes ist eine schwache Andeutung des Si(111)-Reflexes des Substrates bei einem Winkel von $33,1^{\circ}$ zu erkennen. Stark unterschiedliche Intensitäten liegen in der geringen Schichtdicke der laserdeponierten Probe begründet. 
unter einem Winkel von 15,8 , das eine ähnliche Form aufweist wie das entsprechende Maximum einer PMMA-Folie (Abb. 4.10 (b)); auch das zweite, bei der PMMA-Folie unter $35,5^{\circ}$ gut zu erkennende Maximum kann im Diffraktogramm des laserdeponierten Filmes nachgewiesen werden. Die Vergleichbarkeit der beiden Diffraktogramme lässt auf eine ähnliche Nahordnung in Film und bulk-PMMA schließen.

Abbildung 4.11 zeigt ein typisches IR-Transmissionsspektrum einer laserdeponierten PMMA-Schicht, in der zum Vergleich auch ein Spektrum des PMMAAusgangsmaterials dargestellt ist. Im Vergleich der Spektren wird deutlich, dass Film und Target die gleichen Banden mit ähnlichen Absorptionen aufweisen - die che-

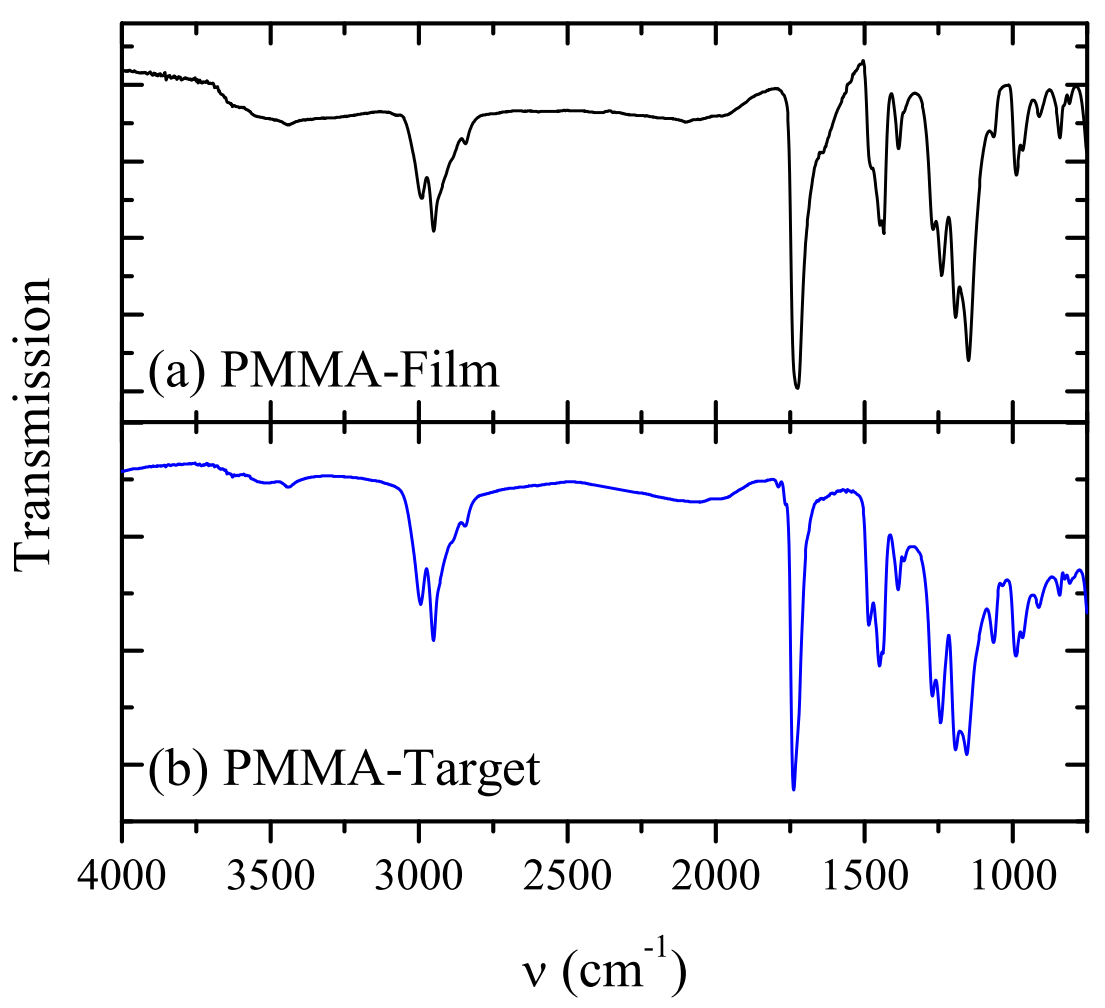

Abbildung 4.11: FTIR-Spektren eines PMMA-Filmes (2000 Pulse, $1500 \mathrm{~mJ} / \mathrm{cm}^{2}$ ) (a) und Vergleichsspektrum des PMMA-Ausgangsmaterials, das durch drop-casting einer PMMA-THF-Lösung hergestellt wurde (b). 
mische Zusammensetzung der Filme repräsentiert daher im Wesentlichen das Ausgangsmaterial. Die Banden sind in Tabelle 4.2 kurz zusammengefasst. Auf Grund des Übertrags von während der Inkubation (vgl. Kap. 3.1) modifizierten Makromolekülen werden jedoch auch einige neue, schwache Banden bei 3078, 2104 und $1643 \mathrm{~cm}^{-1}$, die als $\mathrm{C}=\mathrm{C}$-Doppelbindungen identifiziert werden [58], und eine erhöhte Absorption zwischen 1740 und $1510 \mathrm{~cm}^{-1}$ nachgewiesen. Entsprechend der Absorptionszunahme des Targets [58] weist auch der Film eine vergleichsweise hohe UVAbsorption auf. Abbildung 4.12 zeigt die Absorption vom UV- bis in den sichtbaren

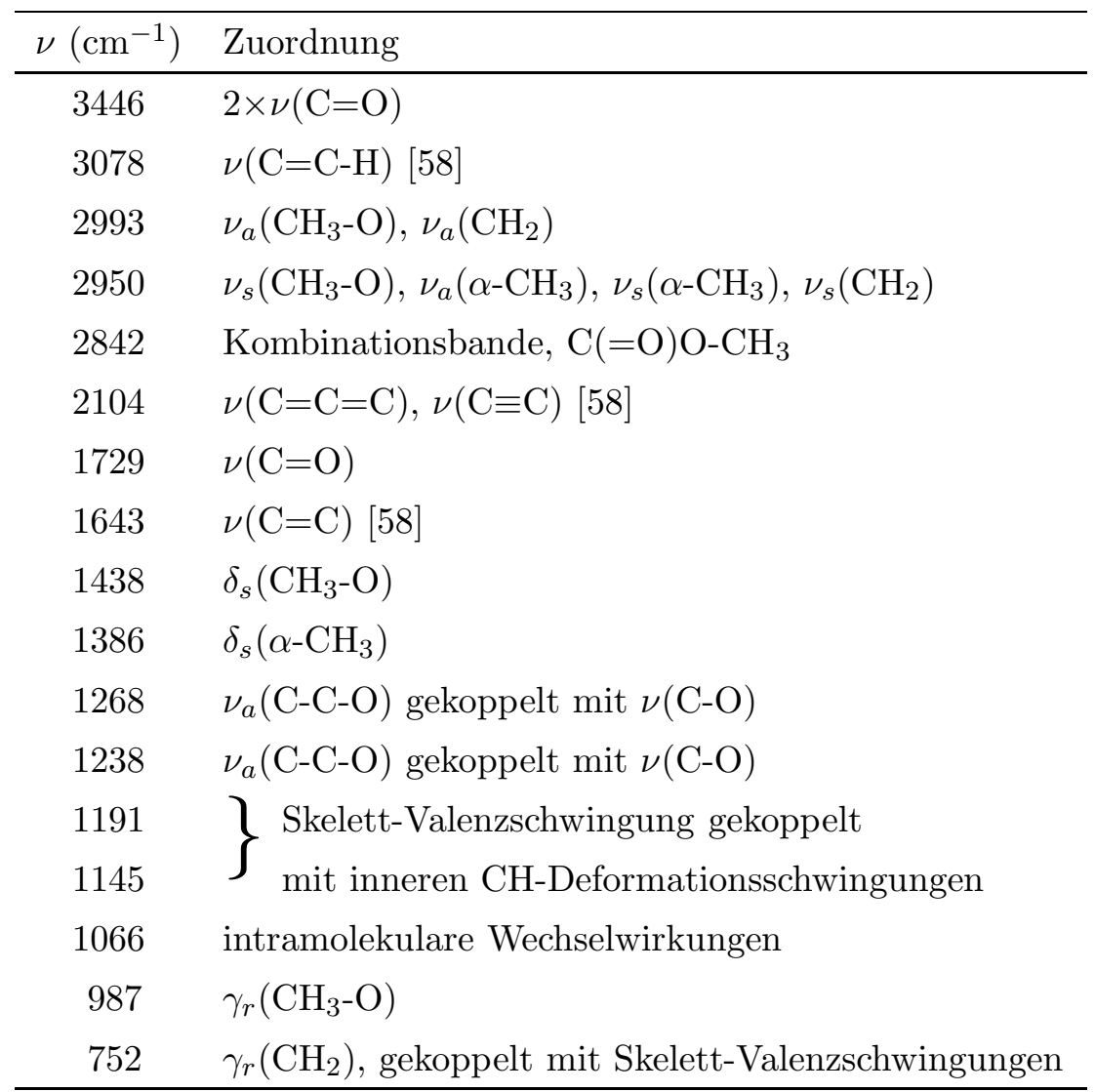

Tabelle 4.2: Experimentell bestimmte Absorptionskanten eines laserdeponierten PMMA-Films (Abbildung 4.11). Die Banden wurden mit der Literatur $[102,20]$ identifiziert. Die Banden bei 3078, 2104 und $1643 \mathrm{~cm}^{-1}$, die in reinem PMMA nicht nachgewiesen werden können, entstehen durch den Ablationsprozess [58]. Bezeichnung der Schwingungen nach Günzler [38]: $\nu_{s / a}$ symmetrische / asymmetrische Streckschwingungen, $\delta / \gamma$ Deformationsschwingungen um den Valenzwinkel / aus der Molekülebene heraus. 


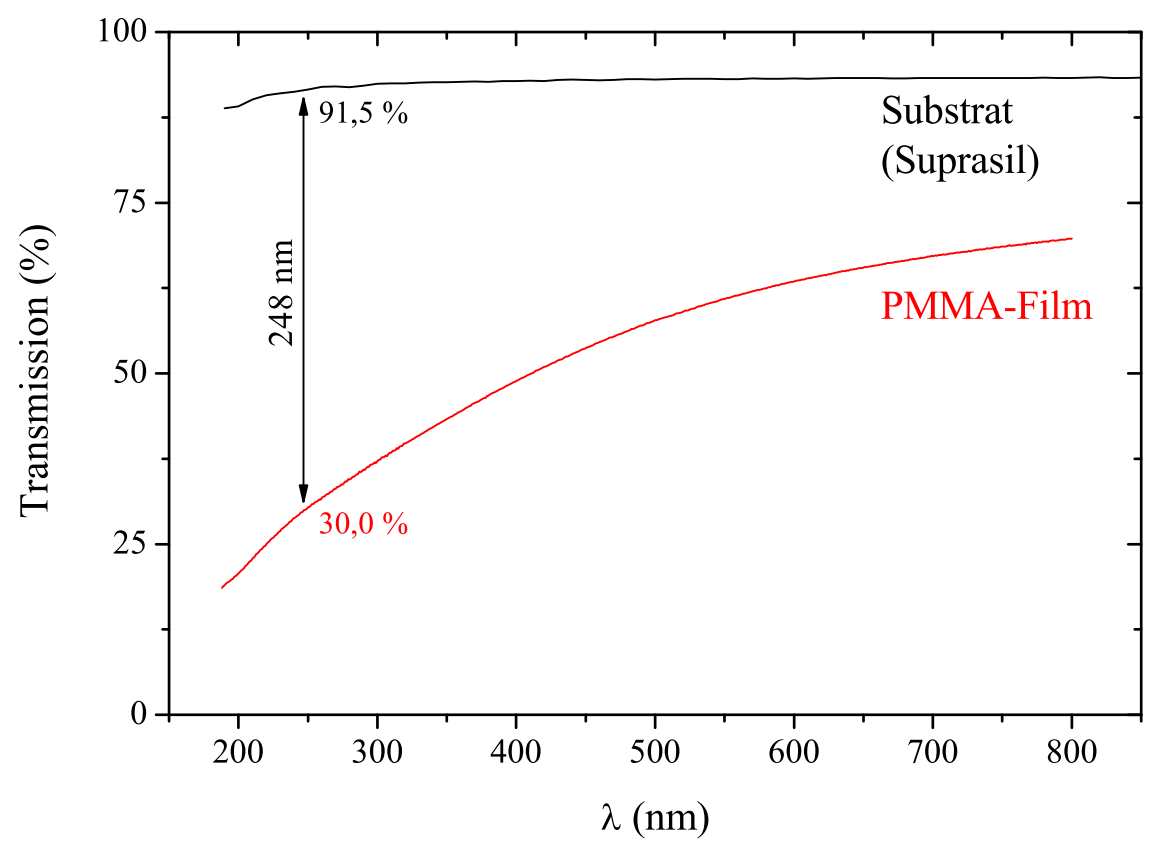

Abbildung 4.12: UV-VIS-Transmissionsspektrum eines PMMA-Filmes (200 Pulse, Energiedichte $1500 \mathrm{~mJ} / \mathrm{cm}^{2}$ ). Zum Vergleich ist auch das Spektrum des Quarzglassubstrates abgebildet.

Wellenlängenbereich anhand eines Transmissionsspektrums eines laserdeponierten PMMA-Films. Aus der Transmission bei 248 nm lässt sich ein Absorptionskoeffizient von etwa $1,5 \cdot 10^{6} \mathrm{~cm}^{-1}$ abschätzen. Die Absorption des Filmes liegt damit zwei Größenordnungen über der Absorption von PMMA [83, 79]. Die damit verbundene gelb-graue Farbe der Filme ist ein weiterer Beweis für die Bildung von Doppelbindungen am Target [62] und nachfolgendem Übertrag von Polymer zum Substrat.

Die chemische Zusammensetzung laserdeponierter PMMA-Filme entspricht im Wesentlichen dem als Tropfen deponierten Targetmaterial. Neben diesem vergleichsweise intakten Polymer existiert eine vernetzte Phase, die in IR-Spektren in Form von veränderten Absorptionsverhältnissen (z.B. bei 1268 oder $1066 \mathrm{~cm}^{-1}$ ) sichtbar wird. 


\subsection{Chemische Zusammensetzung der vernetzten Komponente}

Der vernetzte Anteil laserdeponierter PMMA-Filme entsteht durch die Deposition von Degradationsprodukten, die durch Wechselwirkung der UV-Strahlung mit dem Target entstehen. Aus den Zerfallsmechanismen (vgl. Kap. 3.2, [39, 68, 11]) lassen sich als Zerfallsprodukte im Einklang mit Massenspektren (vgl. Abb. 3.4) im Wesentlichen Moleküle ableiten, die durch Modifikation und Abspaltung der Estergruppe gebildet werden. Bei der Deposition dieser Moleküle auf das Substrat werden chemische Reaktionen, die durch den teilweise radikalischen Charakter der Zerfallsprodukte unterhalten werden, bevorzugt über die Estergruppe ablaufen. Veränderte Absorptionen in den IR-Spektren (vgl. Abb. 4.11, z.B. bei $1268 \mathrm{~cm}^{-1}, \nu_{a}(\mathrm{C}-\mathrm{C}-\mathrm{O})$ ) und eine Zunahme der Absorption zwischen 1740 und $1510 \mathrm{~cm}^{-1}$ deuten auf Modifikationen der Estergruppe im deponierten Film hin [54, 94].

Entsprechend zeigen IR-Spektren des vernetzten Anteils laserdeponierter PMMA-Filme Modifikationen von Banden, die der $\mathrm{OCH}_{3}$-Gruppe zugeordnet werden können. In Abbildung 4.13 sind entsprechende IR-Spektren einer laserdeponierten Probe (bestehend aus Droplets und vernetztem Film) und ihres THF-unlöslichen Rückstandes abgebildet. Deutlich fallen die reduzierten Intensitäten einiger Absorptionsbanden (z.B. bei 1268, 1238, 1191, 846, $752 \mathrm{~cm}^{-1}$ ) auf, während die Bande bei $1729 \mathrm{~cm}^{-1}$ keine nennenswerten Veränderungen zeigt. Die Unterschiede in diesen beiden Spektren sind in Tabelle 4.3 zusammengefasst; gleichzeitig werden dort den Absorptionsbanden Bindungen (nach Willis [102]) zugeordnet. Der Vergleich der beiden Spektren zeigt, dass neben Skelett-Valenzschwingungen, die durch die vernetzte Struktur der Filme eingefroren werden, vorwiegend Banden der $\mathrm{OCH}_{3}$ - und $\mathrm{CH}_{2}$ Gruppen an Absorption verlieren. Die Abnahme der Deformationsschwingungen der $\mathrm{CH}_{2}$-Gruppen ist vor allem auf eine relative Zunahme von $\mathrm{C}=\mathrm{C}$-Doppelbindungen (bei $1643 \mathrm{~cm}^{-1}$ ) zurückzuführen. Die Bildung von Doppelbindungen, die durch die Abspaltung der Estergruppe initiiert wird, entspricht wie die Abspaltung von $\mathrm{OCH}_{3}$ und $\mathrm{CH}_{3}$ aus der Estergruppe der von Gupta [39] beschriebenen photochemischen Zersetzung von PMMA. Die relativen Veränderungen in den IR-Spektren deuten damit im Einklang mit photochemischen Zerfallsmechanismen einen Vernetzungsmechanismus an, der über die $\mathrm{OCH}_{3}$-Gruppe realisiert wird. 


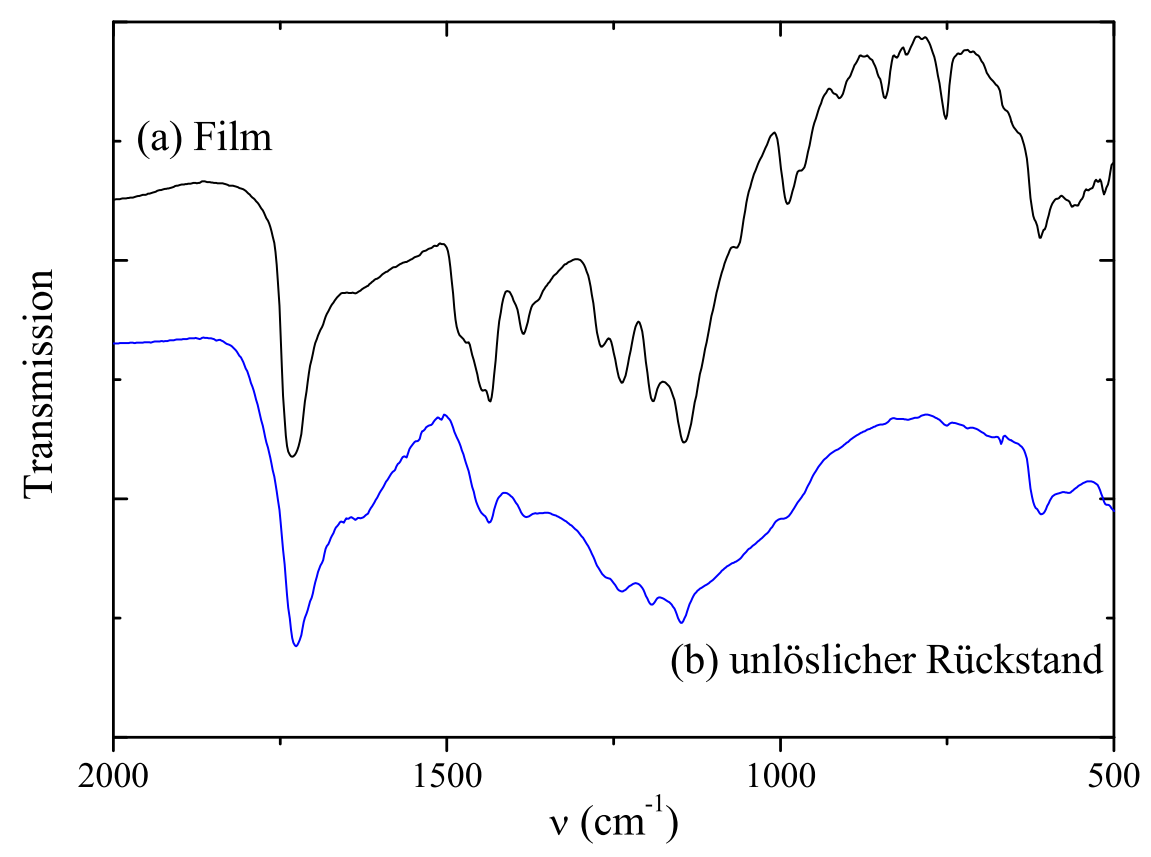

Abbildung 4.13: FTIR-Spektren eines laserdeponierten PMMA-Films (1500 mJ $/ \mathrm{cm}^{2}$, 5000 Pulse, (a)) und des zugehörigen, in THF unlöslichen Rückstandes auf dem Substrat (b). Zur Entfernung des Lösungsmittels aus dem Rückstand wurden die Filme im Vakuum ausgelagert.

Die Vernetzung von PMMA wurde unter etwas geänderten Depositionsbedingungen (XeF-Laser mit einer Wellenlänge von $351 \mathrm{~nm}$, und Anthracen-dotiertes PMMA als Target) beschrieben [96]; allerdings werden hier IR-Spektren beobachtet, in denen die mit $\mathrm{CH}_{2}$ - und $\mathrm{C}=\mathrm{O}$-Gruppen identifizierten Banden abnehmen, woraus auf einen Vernetzungsmechanismus über diese Gruppen geschlossen wird. In den hier gezeigten Spektren kann ein solcher Mechanismus als vorherrschender Prozess ausgeschlossen werden. Die unterschiedlichen Vernetzungsmechanismen sind auf andere Ablationsprodukte bei einer Wellenlänge von $351 \mathrm{~nm}$ zurückzuführen (vgl. Kap. 3). Zusätzlich beigemengtes Anthracen, das zur Steigerung der Absorption des PMMA von Tsuboi verwendet wird, verstärkt diese Unterschiede noch [64].

Auch andere laserdeponierte Systeme wie Polycarbonat (PC) zeigen die Bil- 


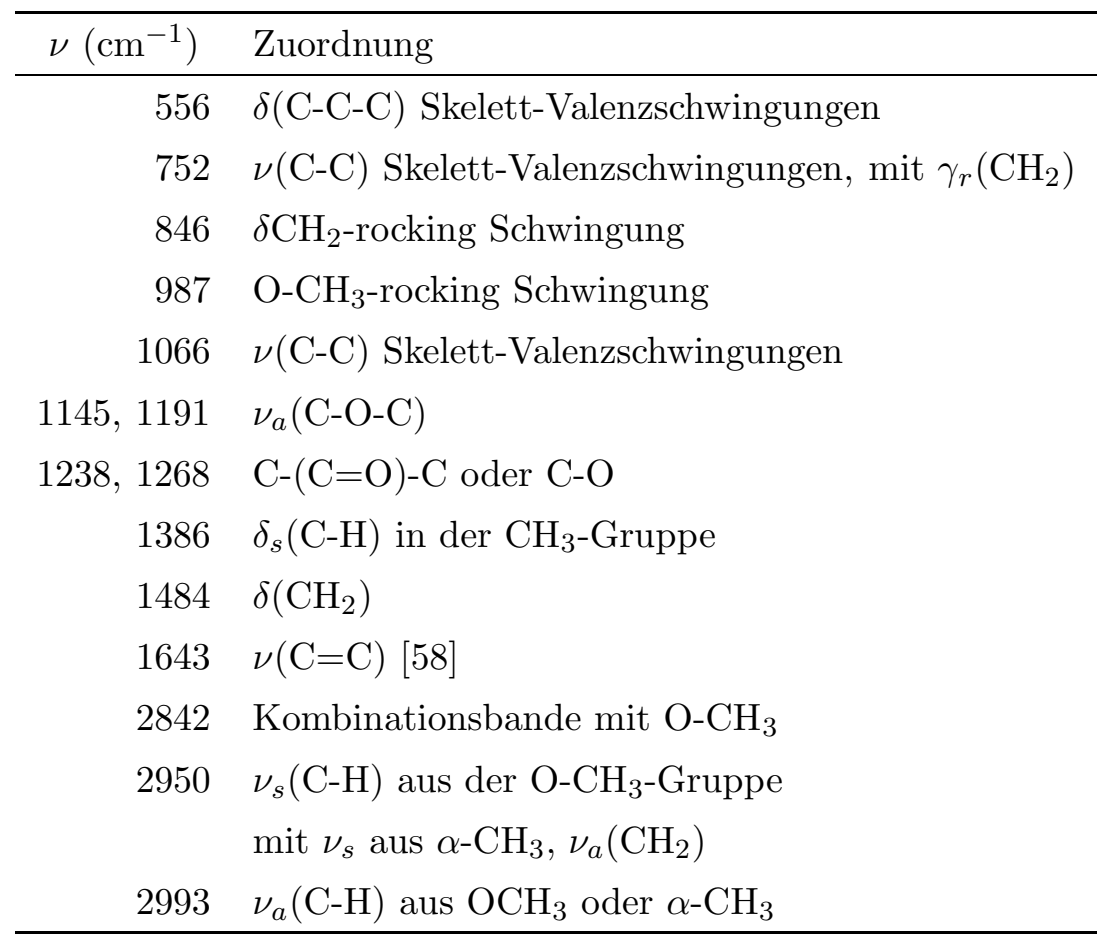

Tabelle 4.3: Position der IR-Banden mit deutlichen Veränderungen zwischen hergestellter Probe und Rückstand (vgl. Abb. 4.13); die Bande bei $1643 \mathrm{~cm}^{-1}$ kann in reinem PMMA nicht nachgewiesen werden, sie ist auf die Bildung von Doppelbindungen zurückzuführen [58]. Die Zuordnung der Banden wurde mit Daten von Willis [102], [20] durchgeführt.)

dung eines Filmes durch vergleichbare chemische Reaktionen von kleinen Degradationsprodukten [98]. Chemische Reaktionen während der Deposition bedingen eine ausreichende Mobilität der Degradationsprodukte auf der Substratoberfläche. Mit Hilfe von Rauigkeitsentwicklungen konnte dieser Aspekt bei der Deposition von PC unter vergleichbaren Bedingungen untersucht werden [40]. Aus dem Übergang vom Einteilchenverhalten bei sehr dünnen Schichten zu kooperativem Schichtwachstum kann auf eine Diffusion kleiner Degradationsprodukte auf der Oberfläche geschlossen werden.

Laserdeponierte PMMA-Filme spiegeln also in ihrer Struktur die zweikomponentige Zusammensetzung der Ablationsprodukte wider. Etwa 65\% des deponierten Filmmaterials besteht aus einem nur leicht modifizierten Polymer mit einer massenmittleren Molmasse von $8 \mathrm{~kg} / \mathrm{mol} ; 15 \%$ des Materials besteht aus Oligomeren mit einer Molmasse zwischen 100 und 1000 g/mol. Diese beiden in organischen Lösungs- 
mitteln löslichen Materialien befinden sich im Wesentlichen in auf das Substrat deponierten Droplets. Neben diesen Droplets liegt ein Anteil von etwa 20\% als vernetzter, durchgehender Film vor. Durch diese Zusammensetzung unterscheidet sich laserdeponiertes PMMA deutlich vom Ausgangsmaterial - und damit genauso von Filmen, wie sie typischerweise mit dem Spincoating-Verfahren hergestellt werden können. Der vernetzte Anteil der Filme ist vergleichbar mit plasmapolymerisierten Filmen.

\subsection{Mechanische Eigenschaften von laserdeponierten PMMA-Filmen}

Auf Grund der besonderen Zusammensetzung, die laserdeponierte PMMA-Filme aufweisen, sind die makroskopischen mechanischen Eigenschaften dieser Filme im Vergleich zum Ausgangsmaterial modifiziert. Während der aus Nanoindentermessungen bestimmte reduzierte Elastizitätsmodul $E_{\mathrm{HU}} /\left(1-\nu^{2}\right)$ (Querkontraktionszahl $\nu \simeq 0$,4) mit 3,2 GPa im Bereich der Modulen für reines Bulk-PMMA liegt (vgl. [104]), nimmt die Härte der Filme stark zu. Abbildung 4.14 stellt die Universalhärte HU eines laserdeponierten Filmes dar; sie nimmt mit steigender Eindringtiefe bis $440 \mathrm{~nm}$ zu, was auf eine raue Oberfläche der Filme zurückzuführen ist: Da die größten Droplets eine Höhe von bis zu 600 nm aufweisen, berührt die pyramidenförmige Spitze zunächst nur einige Droplets und dringt daher nicht vollständig in den Film ein. Bei weiterer Belastung kann die Spitze dann vollständig in den Film eindringen. Dabei fällt die Härte wieder ab.

Bei einer maximalen Eindringkraft von $4 \mathrm{mN}$ wird eine Universalhärte von $62 \mathrm{~N} / \mathrm{mm}^{2}$ erreicht. Dieser Wert liegt deutlich über der Härte des Targets, das nur eine Universalhärte von $12 \mathrm{~N} / \mathrm{mm}^{2}$ zeigt [92]. Bei weiterer Erhöhung der Kraft bleibt die Härte zunächst konstant, bevor sie ab einer Eindringtiefe von $2 \mu \mathrm{m}$ langsam wieder ansteigt. Da dieser Anstieg, der auf den Einfluss des (harten) Si-Substrates zurückzuführen ist, erst bei Eindringtiefen oberhalb von $2 \mu \mathrm{m}$ einsetzt, repräsentieren die bei einer Maximalkraft von $4 \mathrm{mN}$ gemessene Universalhärte und der Elastizitätsmodul im Wesentlichen die mechanischen Eigenschaften des PMMA-Filmes. Mit der vergleichsweise hohen Härte der PMMA-Filme verbunden ist eine schlechte 


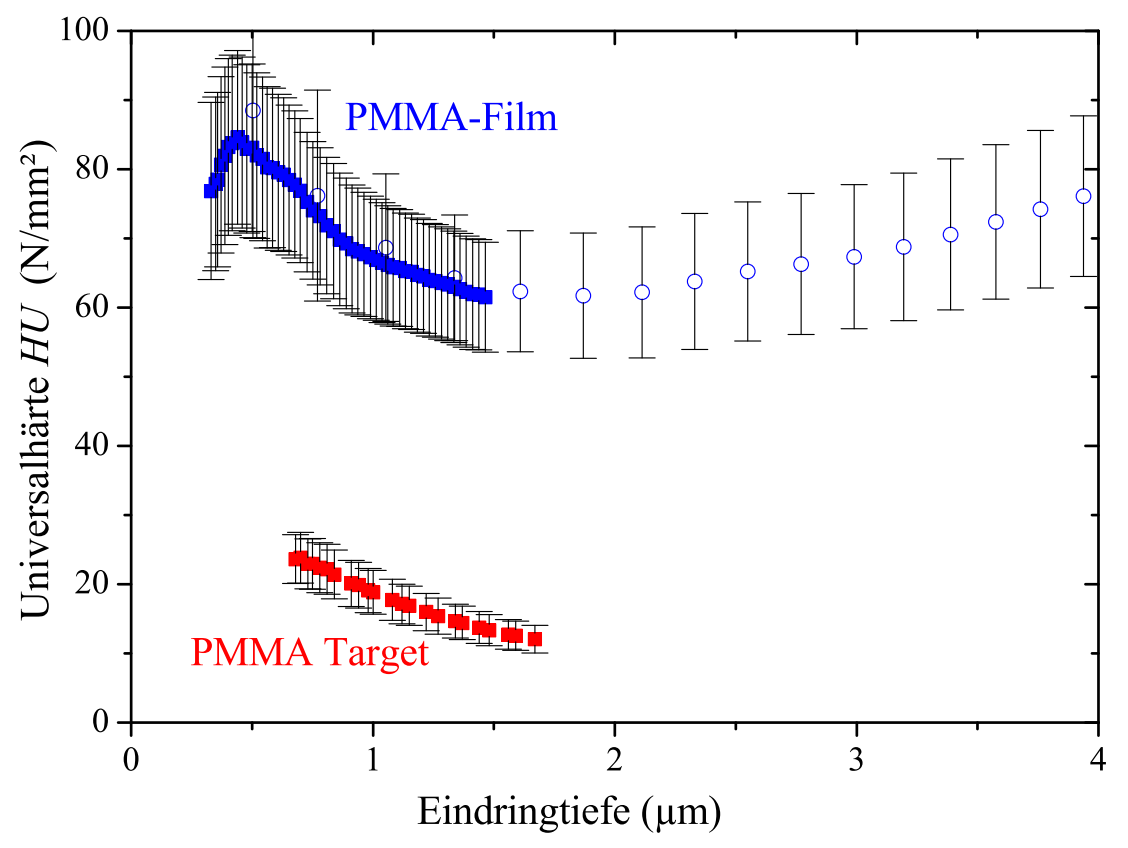

Abbildung 4.14: Universalhärte eines PMMA-Filmes $\left(1500 \mathrm{~mJ} / \mathrm{cm}^{2}\right.$, 20000 Pulse) und des entsprechenden Targets. Die Messreihe mit geschlossenen Symbolen wurde mit einer maximalen Eindringkraft von $4 \mathrm{mN}$ aufgenommen, die offenen Symbole entsprechen einer Maximalkraft von $1 \mathrm{~N}$.

elastische Verformbarkeit der Schichten, die mit etwa 20\% nur einen geringen Anteil - gegenüber $85 \%$ bei PMMA-Folien - an der beim Härteeindruck geleisteten Arbeit einnimmt. Dieser Unterschied ist ein deutlicher Hinweis auf die vernetzte Struktur der Filme.

Die mechanischen Eigenschaften werden damit sowohl durch die Droplets schwach modifizierten PMMAs als auch durch die vernetzte Phase bestimmt: Der Elastizitätsmodul spiegelt ein elastisches Verhalten wider, das einem Polymer entspricht, wohingegen die hohe Härte der Filme auf die Teilvernetzung zurückzuführen ist. Im Vergleich zu idealem PMMA sind noch weitere depositionsbedingte Änderungen der mechanischen Eigenschaften denkbar: Die Deposition von Droplets ist mit einem schnellen Abschrecken der Tröpfchen auf der Substratoberfläche verbunden. Durch das Abschrecken amorpher Materialien wird freies Volumen eingefroren. 
Dadurch müssen Veränderungen wie Defekte oder Spannungen in den Tröpfchen berücksichtigt werden. Anzeichen für diesen Prozess können in Auslagerungsexperimenten gefunden werden, wie sie im Kapitel 7.4 diskutiert werden. Auch eine Ausscheidungshärtung durch Cluster in laserdeponiertem PMMA, wie sie Larciprete [60] vorschlägt, kann nicht ausgeschlossen werden, wenn auch die dort beschriebenen Hinweise auf eine Deposition von Clustern kohlenstoffhaltigen Materials in den hier charakterisierten Proben nicht gefunden wurden.

Zur detaillierteren Charakterisierung der mechanischen Eigenschaften bieten sich weitere Experimente an, die die diskutierten Einflüsse weiter untersuchen: da eine Trennung von polymerer und vernetzter Komponenten auf Grund der makroskopischen Ausdehnung der Nanoindenterprüfspitze nicht gewährleistet werden kann, bieten sich ortsaufgelöste Kraft-Eindringkurven - mit Hilfe der Rasterkraftmikroskopie - an, die eine getrennte Beschreibung von Polymer und vernetztem Schichtanteil ermöglichen. Auch eine Trennung beider Komponenten bei der Deposition mit Hilfe eines mechanischen Filters, wie sie im Kapitel 4.1 diskutiert wurde, bietet die Möglichkeit, den Einfluss von unvernetztem und vernetztem Polymer getrennt zu bestimmen.

Als Ergebnis aus den hier beschriebenen Experimenten lässt sich zusammenfassen, dass laserdeponierte PMMA-Filme aus zwei unterschiedlichen Komponenten bestehen, einem Polymer reduzierter Kettenlänge und einer teilvernetzten Schicht. Beide Komponenten können aus dem Ablationsprozess abgeleitet werden; im eigentlichen Depositionsprozess werden die Ablationsprodukte auf dem Substrat angelagert und bilden dort einen Film. Durch chemische Reaktion entsteht aus kleinen Degradationsprodukte die vernetzte Schicht, während Polymerdroplets durch ein Abschrecken auf dem Substrat erstarren. Durch ein Überwachsen von Droplets mit reaktivem Material wird ein charakteristisches vernetztes Skelett gebildet. Ein Großteil der Schichteigenschaften wird durch Tröpfchen bestimmt, die überwiegend Polymer enthalten, das eine chemisch leicht modifizierte Zusammensetzung bei einer massenmittleren Molmasse von $8300 \mathrm{~g} / \mathrm{mol}$ aufweist. Die Eigenschaften der Schicht, angefangen von der Mikrostruktur über die Zusammensetzung bis hin zu den mechanischen Eigenschaften, spiegeln diese zweikomponentige Zusammensetzung der Schicht deutlich wider. Die gepulste Laserdeposition bietet damit eine Möglichkeit, Schichten herzustellen, die die charakteristische Eigenschaften von unvernetztem Po- 
lymer und einer vernetzten Schicht kombinieren; die hergestellten Schichten weisen Charakteristika auf, die zwischen den Eigenschaften eines unvernetzten, mit Hilfe eines Spincoating-Verfahrens hergestellten Filmes auf der einen Seite, und - auf der anderen Seite - einer durch Plasmapolymerisation (z.B. [5, 80]) hochgradig vernetzten Schicht liegen. 


\section{5}

\section{Einfluss der Energiedichte auf die Zusammensetzung}

Die Eigenschaften laserdeponierter PMMA-Filme hängen wesentlich von der Zusammensetzung der Filme ab, die, wie im Kapitel 4 beschrieben, aus zwei Komponenten bestehen. Zur Modifikation der Eigenschaften ist es notwendig, diese Komponenten zu verändern. Eine Variationsmöglichkeit bietet hierbei der Ablationsmechanismus. Ein Eingriff in die Ablation erfordert eine Veränderung der Wechselwirkungen zwischen Laser und Targetmaterial, die wesentlich durch die Absorption ultravioletter Strahlung im Polymer bestimmt werden. Eine Möglichkeit bietet hier die Verwendung eines Lasers mit veränderter Wellenlänge [19] oder Pulsdauer [57]; eine andere Möglichkeit besteht darin, das Targetmaterial mit einem Absorptionsverstärker zu dotieren [33, 64]. Ohne großen experimentellen Aufwand kann der Ablationsprozess in Grenzen jedoch auch durch die Veränderung der Energiedichte variiert werden. Daher wird in diesem Kapitel der Einfluss der Energiedichte auf die Eigenschaften laserdeponierter PMMA-Filme untersucht.

\subsection{Molmassenverteilung von Droplets und Oligomeren}

Die Zusammensetzung der Filme ist eng mit dem Ablationsmechanismus verbunden, in dem die Produkte - wie im Kapitel 3.2 diskutiert - durch eine Volumenexplo- 
sion abgetragen werden. Aus der Literatur ist bekannt, dass eine Veränderung der Energiedichte sich am Target wesentlich auf die Degradation des Polymers zu kleinen Produkten auswirkt, in dem bei steigender Energiedichte mehr kleine Produkte gebildet werden $[83,9]$.

Abbildung 5.1 zeigt SE-Chromatogramme laserdeponierter PMMA-Proben, die eine - unabhängig von der Energiedichte - konstante massenmittlere Molmasse von $8 \mathrm{~kg} / \mathrm{mol}$ aufweisen. Das unvernetzte, in Droplets deponierte Polymer zeigt keine signifikanten Veränderungen bei einer Variation der Energiedichte, wohl aber der Anteil der Oligomere, die durch eine Polymerisation von Monomeren auf dem Substrat gebildet werden, mit einer Masse von 114, 171, 228, 318, 522 und 843 g/mol. Entsprechend einer zunehmenden Degradation des Polymers in kleine Moleküle steigt die Konzentration dieser Oligomere mit der Energiedichte an, sichtbar am systematischen Anstieg der Chromatogramme bei kleinen Molmassen. Abbildung 5.2 (a)

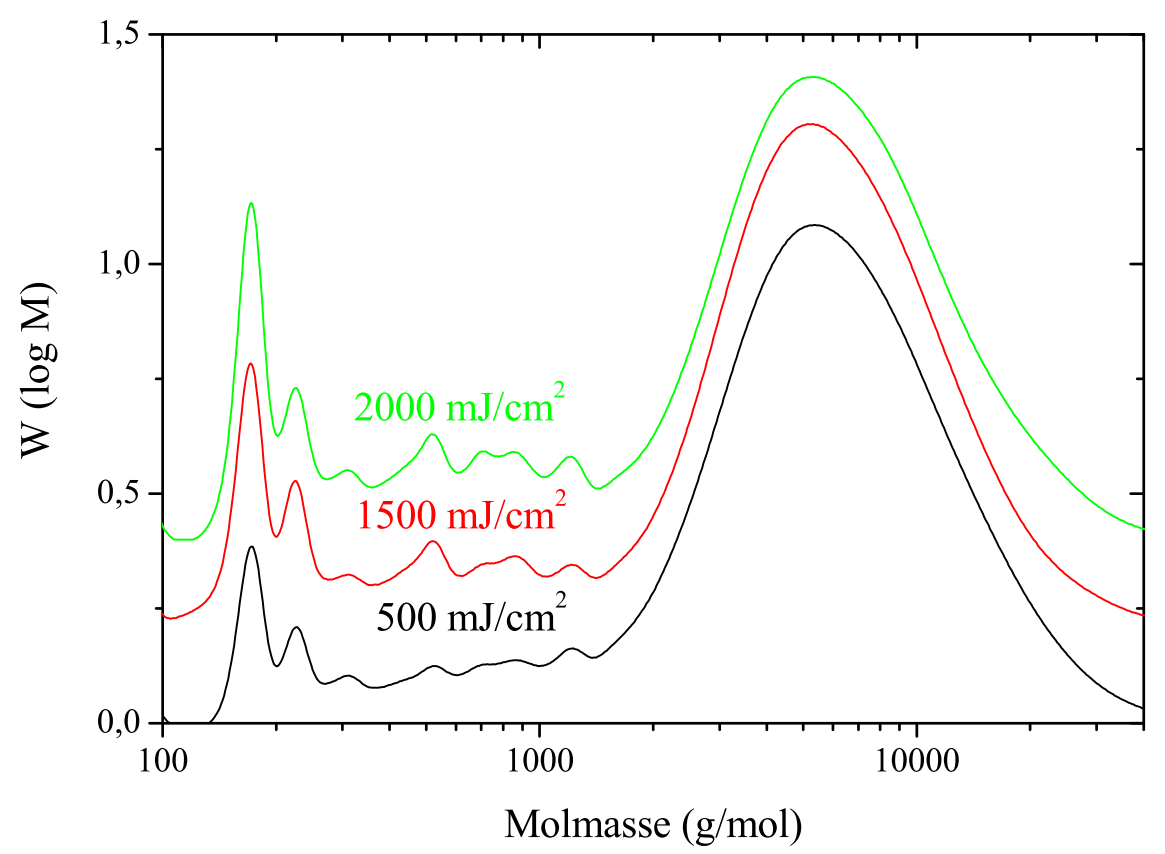

Abbildung 5.1: SE-Chromatogramm des in THF löslichen Anteils verschiedener PMMA-Filme, die bei unterschiedlichen Energiedichten von 500$2000 \mathrm{~mJ} / \mathrm{cm}^{2}$ hergestellt wurden (20000 Pulse, Target: $\mathrm{M}_{n}>4 \cdot 10^{6} \mathrm{~g} / \mathrm{mol}$ ). 


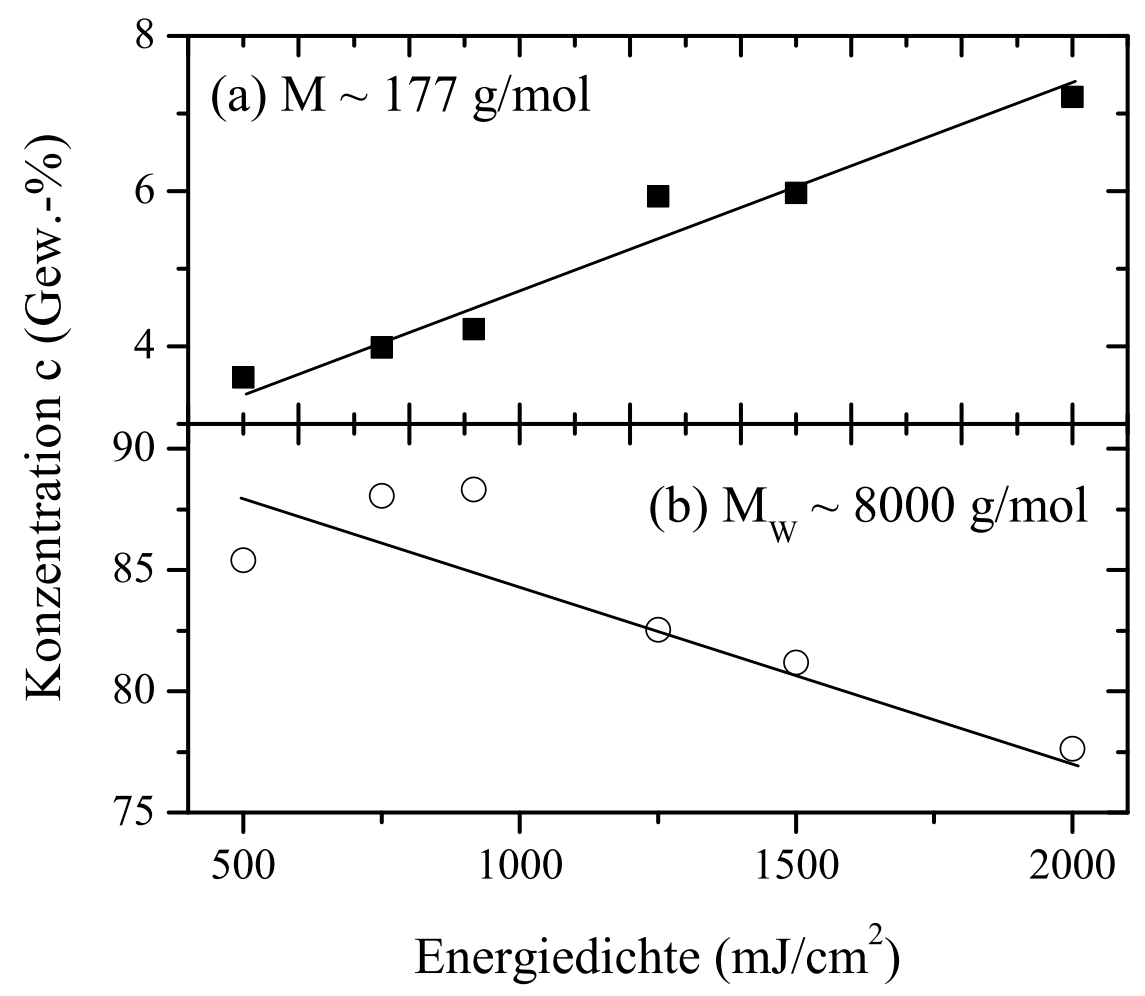

Abbildung 5.2: Gewichtskonzentration der Moleküle mit einer molaren Masse von $177 \mathrm{~g} / \mathrm{mol}$ als Funktion der Energiedichte (a), bestimmt über eine Anpassung der Chromatogramme (5.1) mit Gaußfunktionen und anschließender Integration, und Konzentration des Polymeranteils $\left(\mathrm{M}_{W} \simeq 8000 \mathrm{~g} / \mathrm{mol}\right)$ (b).

stellt diesen Anstieg anhand der Gewichtskonzentration von Molekülen mit einer Molmasse von $177 \mathrm{~g} / \mathrm{mol}$ dar. Damit verbunden ist auch eine Abnahme der Konzentration großer Moleküle $(\mathrm{M}>2000 \mathrm{~g} / \mathrm{mol})$, die von 87 Gew.- $\%$ bis auf 77 Gew.- $\%$ abfällt (Abb. $5.2(\mathrm{~b}))$. 


\subsection{Veränderung der chemischen Zusammensetzung}

Eine Variation der Energiedichte hat keinen Einfluss auf die Molmasse der Droplets, wohl aber auf den Anteil des unvernetzten Polymers an der Gesamtschicht, der mit steigender Energiedichte abnimmt. Da dieser durch eine Erhöhung der Energiedichte reduziert werden kann, wirkt sich die Energiedichte auch auf die Zusammensetzung aus.

Wie Abbildung 5.3 anhand von IR-Spektren vergleichbarer PMMA-Schichten zeigt, die bei unterschiedlichen Energiedichten von $900 \mathrm{~mJ} / \mathrm{cm}^{2}$ bis $2200 \mathrm{~mJ} / \mathrm{cm}^{2}$ hergestellt wurden, äußert sich diese Änderung durch eine zunehmende Abweichung vom idealen PMMA-Spektrum. Entsprechend der Struktur der vernetzten Komponente lässt sich die Veränderung der Zusammensetzung vor allem an Banden, die mit

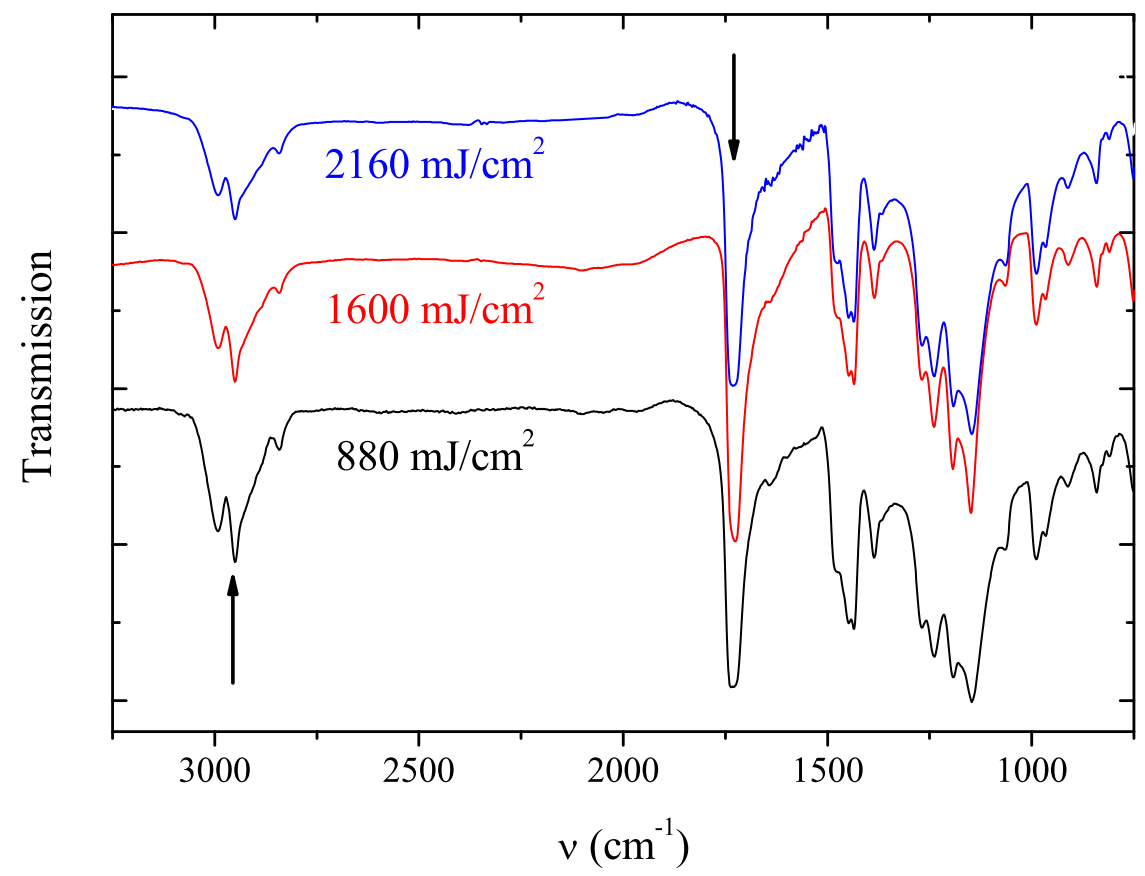

Abbildung 5.3: FTIR-Spektren von PMMA-Filmen, die unter unterschiedlichen Laserenergiedichten hergestellt wurden. 
der $\mathrm{OCH}_{3}$-Gruppe im Ester in Verbindung stehen, festmachen (vgl. Kap. 4.4). Es kann eine mit steigender Energiedichte fallende Absorption beispielsweise anhand der C-C-O-Banden bei 1268 oder $1238 \mathrm{~cm}^{-1}$ beobachtet werden. Deutlicher wird dieser Unterschied in der Absorption der Banden zwischen 3000 und $2842 \mathrm{~cm}^{-1}$, die als C-H-Streckschwingungen der $\mathrm{CH}_{3}-\mathrm{O}-, \mathrm{CH}_{2^{-}}, \mathrm{CH}_{3^{-}}$und $\mathrm{C}(=\mathrm{O}) \mathrm{OCH}_{3}-\mathrm{Gruppen}^{-}$ identifiziert werden können [102].

Abbildung 5.4 zeigt die Absorption dieser Banden bezogen auf die Absorption der Carbonylbande $\left(1730 \mathrm{~cm}^{-1}\right)$ als einer durch den Vernetzungsprozess unveränderten Bande. Das Verhältnis dieser Banden fällt mit steigender Energiedichte ab und

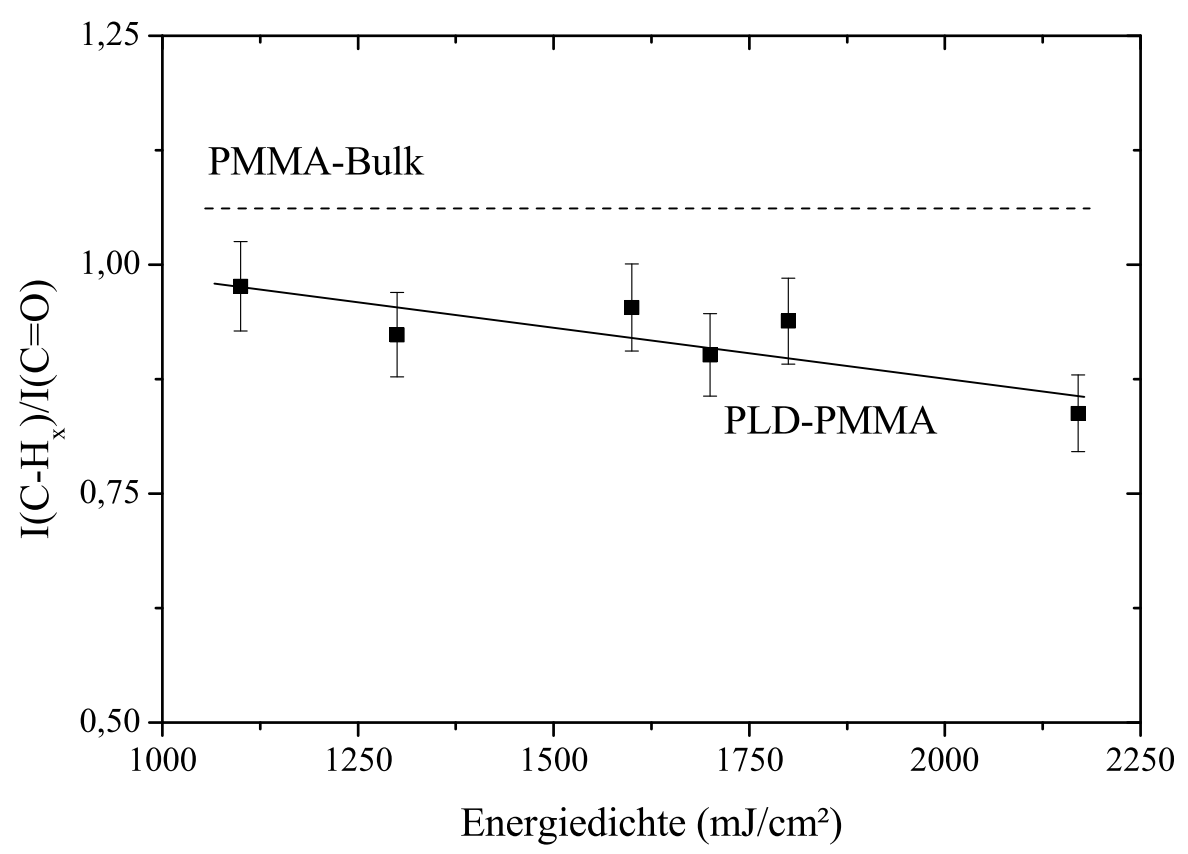

Abbildung 5.4: Verhältnis der Carbonyl- und C-H-Streckschwingungen. Die Intensitäten der Banden wurden durch Integration über die FTIR-Spektren in der Abbildung 5.3 zwischen 2856 und $3062 \mathrm{~cm}^{-1}(\mathrm{CH})$ und 1652 und $1750 \mathrm{~cm}^{-1}(>\mathrm{C}=\mathrm{O})$ bestimmt. Durch den Vergleich der Bandenabsorption können experimentelle Ungenauigkeiten wie variierende Schichtdicken oder unterschiedliche IR-Intensitäten des Spektrometers weitestgehend ausgeglichen werden. 
kann folglich als einfaches Maß für den Anteil von polymerer zu vernetzter Komponente betrachtet werden.

Mit einer Variation der Energiedichte kann also die Zusammensetzung der Ablationsprodukte verändert werden. Die zunehmende Energiedichte modifiziert dabei nicht die Produkte selbst, d.h. es werden weiterhin Droplets mit modifiziertem Polymer und kleine, photochemisch aus PMMA gebildete Degradationsprodukte ablatiert. Stattdessen wird die Verteilung der Produkte verändert: Sowohl die SEChromatogramme als auch FTIR-Spektren zeigen eine Zunahme von Produkten, die eine vom polymeren Anteil unterschiedliche Molmasse bzw. modifizierte Zusammensetzung aufweisen. Die Reduktion des unvernetzten polymeren Anteils ist bei beiden Methoden vergleichbar: Durch eine Erhöhung der Energiedichte von $1 \mathrm{~J} / \mathrm{cm}^{2}$ auf $2 \mathrm{~J} / \mathrm{cm}^{2}$ fällt der Anteil des unvernetzten Polymer um 10-15\% ab. Damit verbunden ist eine relative Zunahme der vernetzten Struktur. Durch eine Variation der Energiedichte wird daher der Vernetzungsgrad laserdeponierter Filme verändert. Als weiterführende Experimente bieten sich z.B. Festkörper-NMR-Analysen an, die diesen Vernetzungsgrad quantitativ beschreiben können.

\subsection{Modifikation der mechanischen Eigenschaften}

Die mechanischen Eigenschaften laserdeponierter PMMA-Filme werden wesentlich durch das Nebeneinander von vernetzter Komponente und unvernetzten Polymerdroplets bestimmt (vgl. Kap. 4.5). Mit der Zunahme der vernetzten Komponente mit der Energiedichte ist eine Verfestigung der Filme verknüpft, wie in Abhängigkeit der Energiedichte in Abbildung 5.5 anhand des reduzierten Elastizitätsmoduls $E_{\mathrm{HU}} /\left(1-\nu^{2}\right)$ dargestellt ist; der Elastizitätsmodul steigt von 2,4(2) GPa bei einer Energiedichte von $1 \mathrm{~J} / \mathrm{cm}^{2}$ auf 4,8(5) GPa bei $2,5 \mathrm{~J} / \mathrm{cm}^{2}$ an.

\subsection{Vergleich mit Modellsystem PVCin: Verfestigung mit zunehmender Vernetzung}

Laserdeponiertes PMMA zeigt eine Verfestigung der Filme mit zunehmendem Anteil der vernetzten Komponente. Um diese Abhängigkeit der mechanischen Eigen- 


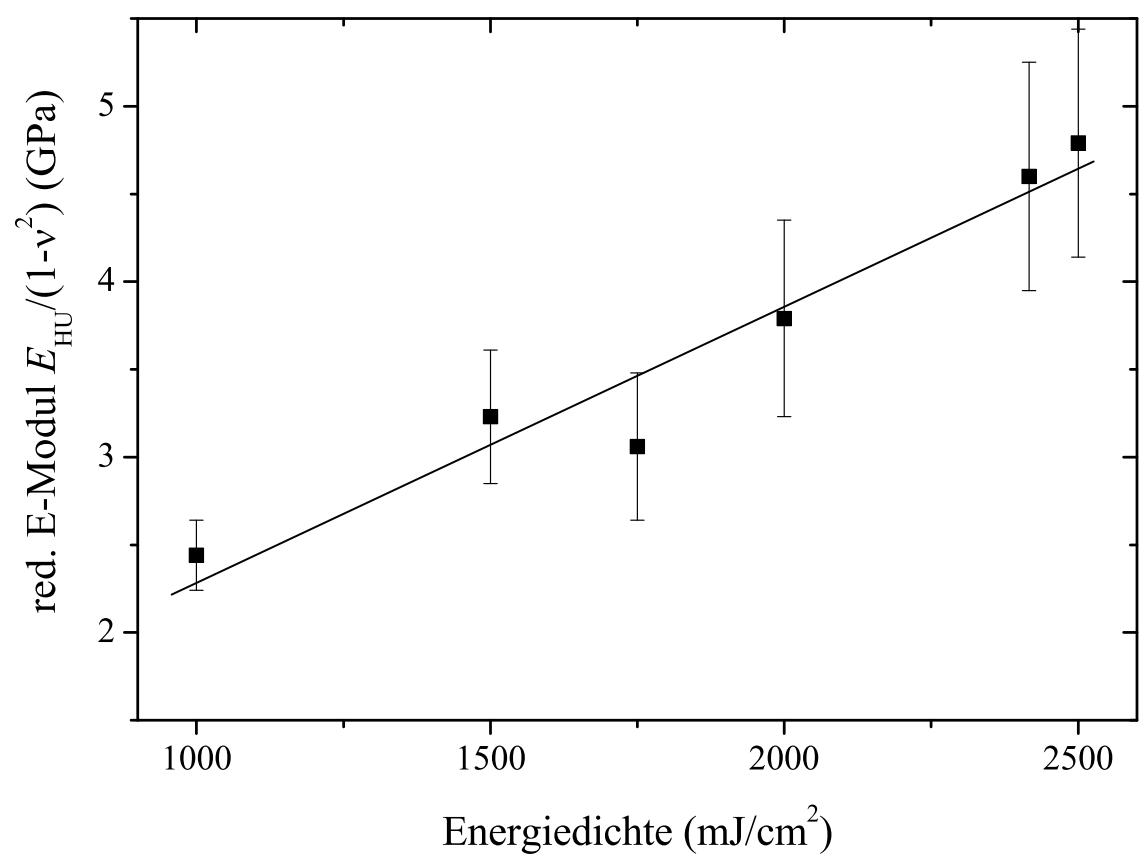

Abbildung 5.5: Reduzierter E-Modul von laserdeponierten PMMA-Schichten als Funktion der Energiedichte.

schaften vom Vernetzungsgrad detaillierter zu untersuchen, wurden entsprechende Experimente mit Poly(vinyl-zimtsäureester), PVCin, durchgeführt, das sich als Modellsystem anbietet, da es durch eine UV-Bestrahlung systematisch vernetzt werden kann. Dieser Vernetzungsprozess wird in Abbildung 5.6 skizziert. Der Grad der Vernetzung hängt von der Bestrahlungszeit ab [15].

Dazu wurden in Zusammenarbeit mit der Gruppe Polymerphysik am Institut für Materialphysik Filme auf Si- oder Polyimid-(PI)-Substrate aufgetropft und sukzessive mit einer Hg-Dampflampe belichtet. Durch Messung der IR-Transmission der an der Vernetzung beteiligten Gruppen [69] kann der Vernetzungsgrad des Polymers bestimmt werden. Parallel wurden die mechanischen Eigenschaften des PVCin am Beispiel des reduzierten Elastizitätsmoduls mit Hilfe des Nanoindenters untersucht.

Abbildung 5.7 (a) zeigt FTIR-Spektren von PVCin-Filmen für unterschiedliche Bestrahlungszeiten. Diese Spektren unterscheiden sich in den Absorptionen 


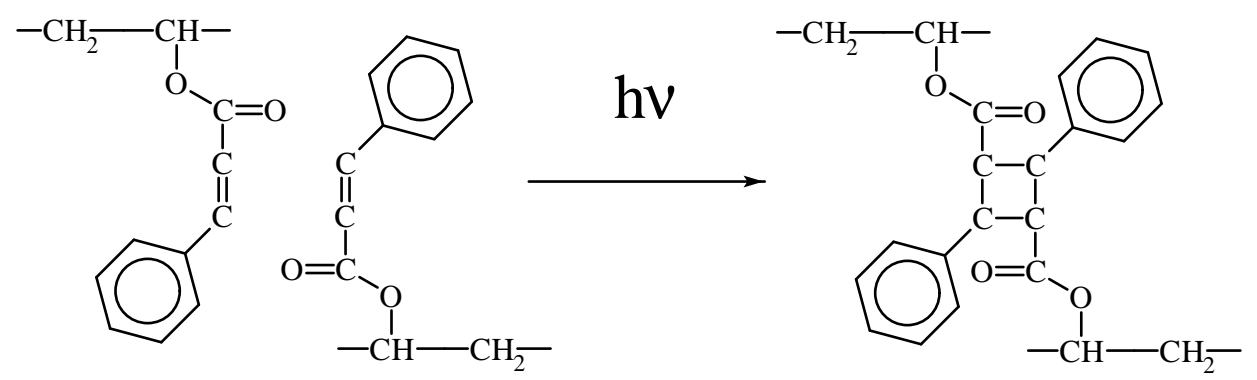

Abbildung 5.6: Schematische Vernetzungsreaktion von Poly(Vinyl Zimtsäureester). Durch die UV-Bestrahlung wird eine Estergruppe angeregt; die Vernetzung wird über eine Photoaddition dieser Gruppe mit einer Gruppe im Grundzustand realisiert.

der $\mathrm{C}=\mathrm{C}$-Bande bei $1635 \mathrm{~cm}^{-1}$ (1), die mit zunehmender Bestrahlungsdauer abfällt, und der Absorption der C=O-Gruppe des ungesättigten Esters $\left(1714 \mathrm{~cm}^{-} 1,(2)\right)$, die abfällt und sich zur Schwingung des gesättigten Esters $\left(1750 \mathrm{~cm}^{-1},(3)\right)$ hin verschiebt, wie man aus dem Vernetzungsprozess (vgl. Abb. 5.6) erwartet. Ein Maß für den Vernetzungsgrad ist die Intensität der Doppelbindung [69]. Um experimentelle Ungenauigkeiten auszugleichen, wurden diese Absorptionen, bestimmt über eine Integration, mit denen der CH-Streckschwingungen als von der Vernetzung unbeeinflusste Referenzbande verglichen. Dieses Intensitätsverhältnis ist in Abbildung 5.7 (b) für unterschiedliche Proben und unterschiedliche Bestrahlungszeiten dargestellt. Klar kann ein exponentieller Abfall der Intensität bis auf etwa 20\% des unbehandelten Ausgangsmaterials nach einer Bestrahlungszeit von 120 min beobachtet werden. Die verbliebene Absorption der Doppelbindung kann auf eine unvollständige Vernetzung in Folge zu kurzer Bestrahlungszeiten oder auf eine Vernetzungsprozess, bei dem nicht jede Doppelbindung zur Vernetzung beiträgt, hindeuten. Eine mögliche Erklärung für dieses Verhalten liegt in der räumlichen Entfernung der am Vernetzungsprozess beteiligten Doppelbindungen, die sich bei einer chemischen Reaktion in einem Abstand von weniger als 150 pm befinden müssen.

Verbunden mit der Vernetzung von PVCin ist eine Verfestigung des Polymers. In Abbildung 5.8 (a) ist der reduzierte Elastizitätsmodul einer PVCin-Schicht als Funktion der Beleuchtungszeit dargestellt. Der Elastizitätsmodul steigt von 4,2 auf 


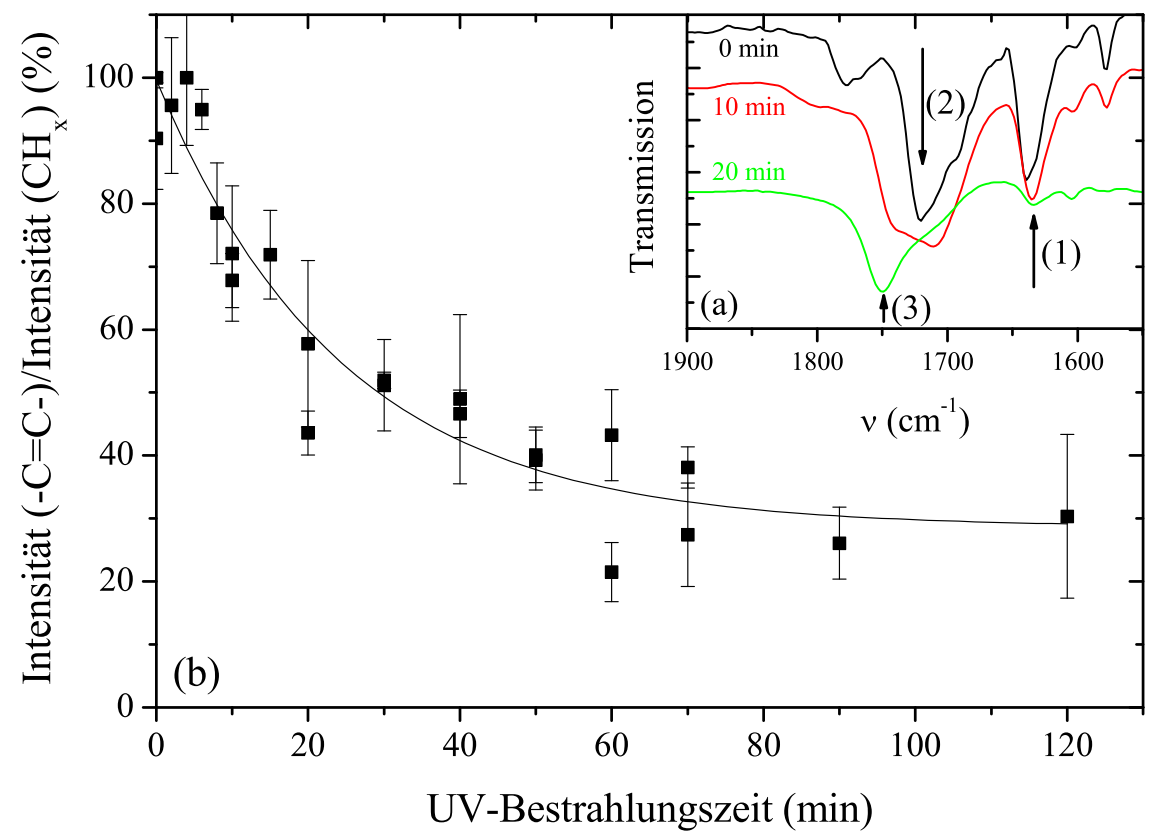

Abbildung 5.7: FTIR-Spektren von PVCin-Filmen nach unterschiedlichen Bestrahlungszeiten (a) und Verhältnis der $\mathrm{C}=\mathrm{C}$-Schwingungen zu $\mathrm{CH}$ Streckschwingungen als Funktion der UV-Bestrahlungszeit, bezogen auf die Absorptionen der unbehandelten Probe (b). Dieses Verhältnis kann als Maß für den Vernetzungsgrad des PVCin angenommen werden.

4,6 GPa nach 60 min UV-Bestrahlung an. Diese Verfestigung ist etwa proportional zur Absorption der Doppelbindungen, wie in Abbildung 5.8 (b) gezeigt wird. Ähnliche Ergebnisse konnten auch in der mechanischen Spektroskopie gefunden werden, wo mit der Bestrahlungszeit auch Glasübergangstemperatur und der Elastizitätsmodul zunehmen [43].

Insgesamt konnte gezeigt werden, dass die mechanischen Eigenschaften von Polymerfilmen systematisch von der Vernetzung abhängen. Über eine Veränderung der Energiedichte bietet sich bei der gepulsten Laserdeposition von PMMA die Möglichkeit, mit dem Ablationsprozess die Verhältnisse von kleinen Degradationsprodukten zu Polymerdroplets zu verändern. Diese Verschiebung der Anteile schlägt sich auch in der Deposition nieder, indem der Einfluss der vernetzten Komponente zunimmt. Dies 


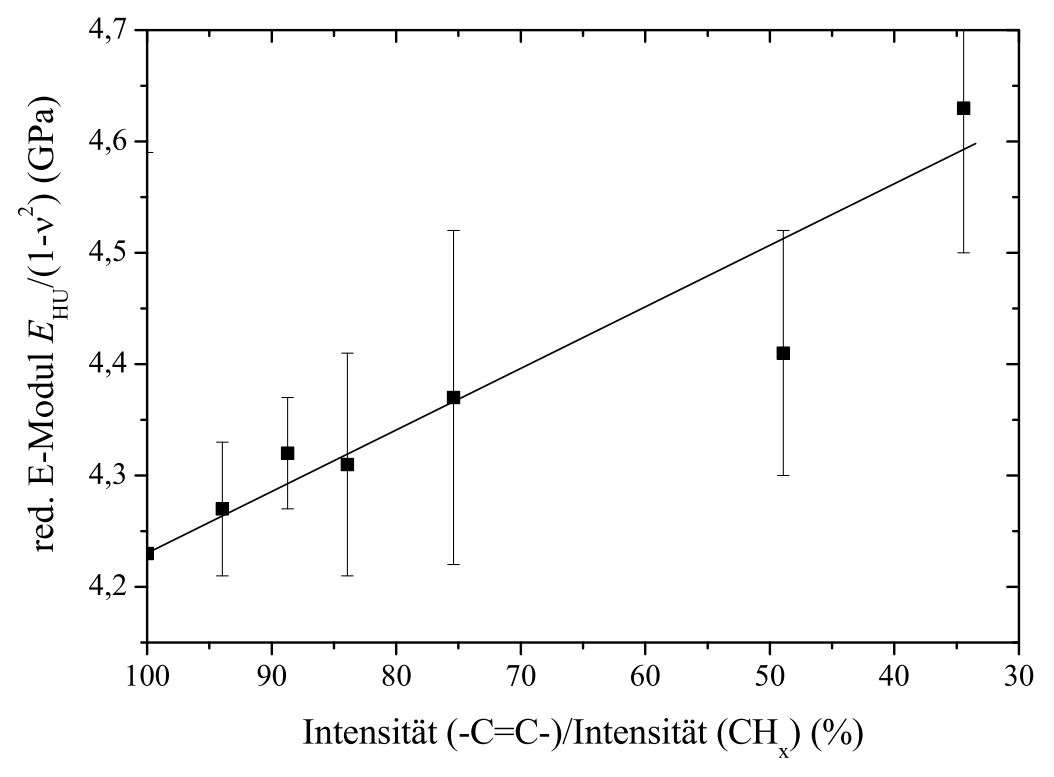

Abbildung 5.8: Reduzierter E-Modul von PVCin-Schichten als Funktion des Verhältnises der $\mathrm{C}=\mathrm{C}$-Schwingungen zu $\mathrm{CH}-$ Streckschwingungen als Maß des Vernetzungsgrades.

führt zu systematisch modifizierten Filmeigenschaften. Eine Variation der Energiedichte kann daher zur gezielten Einstellung von mechanischen Eigenschaften genutzt werden. 


\section{6}

\section{Einfluss der Substrattemperatur auf die Schichteigenschaften}

Zur Veränderung der Schichteigenschaften bietet sich als Alternative zum Eingriff in den Ablationsprozess eine Modifikation der ablatierten Produkte während der Deposition auf dem Substrat an: Durch eine Deposition auf geheizte Substrate können thermisch aktivierte Prozesse angeregt werden. Eine zusätzliche Energiezufuhr auf die Produkte sollte den Depositionsprozess grundlegend verändern. In diesem Kapitel daher wird der Einfluss der Substrattemperatur auf Schichtcharakteristika wie Mikrostruktur und Molmasse untersucht.

\subsection{Veränderung der Oberfläche}

Die Mikrostruktur laserdeponierter PMMA-Filme entsteht durch Abschrecken flüssiger Droplets bei der Deposition auf das Substrat. Die Zufuhr von thermischer Energie durch Erhöhen der Substrattemperatur reduziert diesen Abkühlprozess und verlängert somit die Zeit, in der sich die Droplets über der Glasübergangstemperatur befinden. Bei einer Molmasse von 5000-8000 g/mol liegt diese bei etwa $90-100{ }^{\circ} \mathrm{C}$ [73]. Oberhalb davon verhält sich das Polymer wie eine viskose Flüssigkeit und die Mobilität der Makromoleküle reicht aus, um Droplets teilweise glatt zu ziehen.

Abbildung 6.1 zeigt diesen Prozess anhand von elektronenmikroskopischen Aufnahmen: Die Morphologie von PMMA-Filmen, die bei Raumtemperatur deponiert wurden, wird wesentlich durch Droplets bestimmt, die kugelförmig-zylindrische In- 


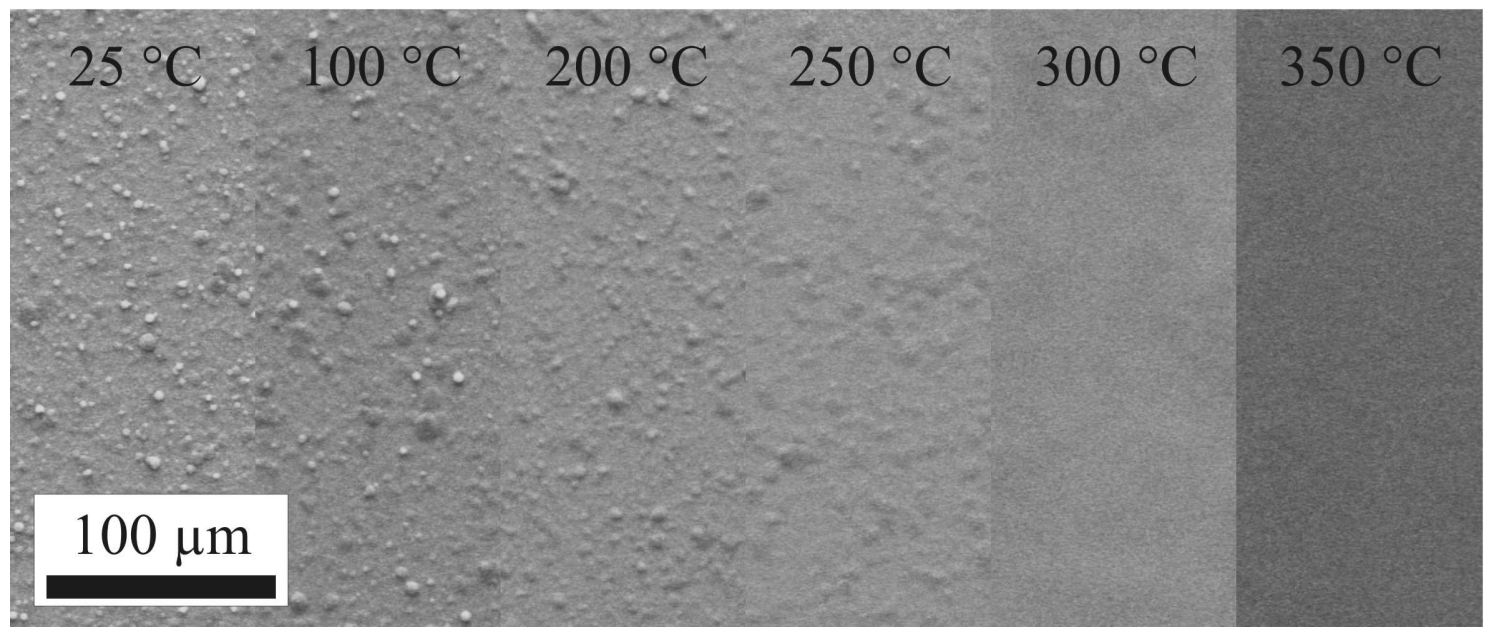

(a) Aufnahmen verschiedener PMMA-Filme

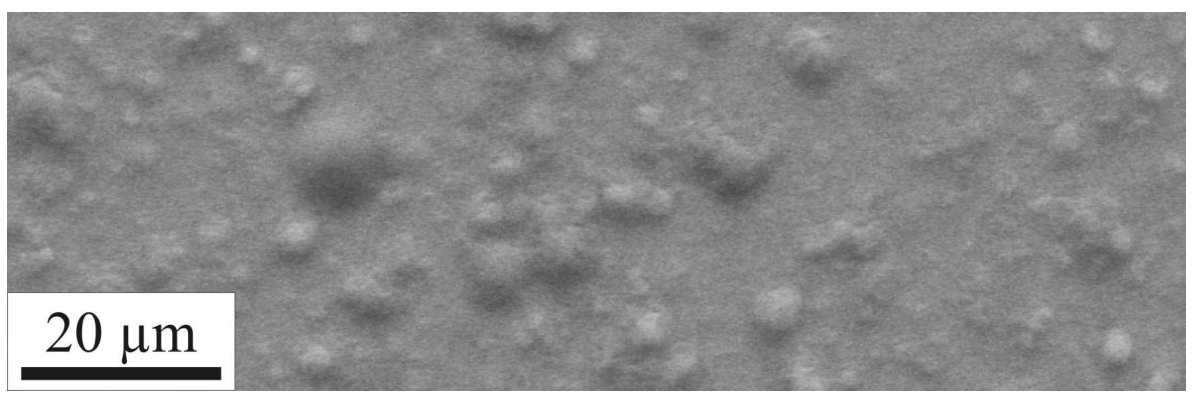

(b) Substrattemperatur $200{ }^{\circ} \mathrm{C}$

Abbildung 6.1: Elektronenmikroskopische Aufnahmen von PMMA-Filmen, die bei unterschiedlichen Substrattemperaturen von $25{ }^{\circ} \mathrm{C}$ bis $350{ }^{\circ} \mathrm{C}$ hergestellt wurden (1500 mJ $/ \mathrm{cm}^{2}, 5000$ Pulse) (a) und Ausschnitt eines Filmes, der bei $200{ }^{\circ} \mathrm{C}$ deponiert wurde (b).

seln bilden. Mit steigender Substrattemperatur verlaufen diese Tröpfchen, so dass die Inseln glockenförmige Erhebungen bilden, im Ausschnitt in Abbildung 6.1 (b) zu sehen. Entsprechend der Glastemperatur können diese auseinandergeflossenen Droplets bei Substrattemperaturen oberhalb von $100{ }^{\circ} \mathrm{C}$ beobachtet werden. Durch das Glattziehen der Droplets nimmt die Rauigkeit der Filme mit steigender Temperatur ab, so dass die Filme oberhalb von $250{ }^{\circ} \mathrm{C}$ eine im Rasterelektronenmikroskop kontrastarme Mikrostruktur aufweisen. Unter leicht veränderten Depositionsbedin- 
gungen (Nd-YAG-Laser, 266 nm, 10 ns) beschreiben Blanchet [9] oder Wee [101] eine ähnliche Glättung von laserdeponierten PMMA-Filmen mit steigender Substrattemperatur.

\section{2 Änderung der Molmasse des deponierten Polymers}

Die Zufuhr zusätzlicher Energie kann auch zur Anregung von thermisch aktivierten chemischen Reaktionen genutzt werden. Innerhalb der Polymerdroplets sind so Reaktionen möglich, die zu Makromolekülen erhöhter Molmasse führen. In Abbildung 6.2 sind Chromatogramme von Filmen dargestellt, die bei unterschiedlichen Temperaturen aufgewachsen wurden. Die Molmassenverteilung der

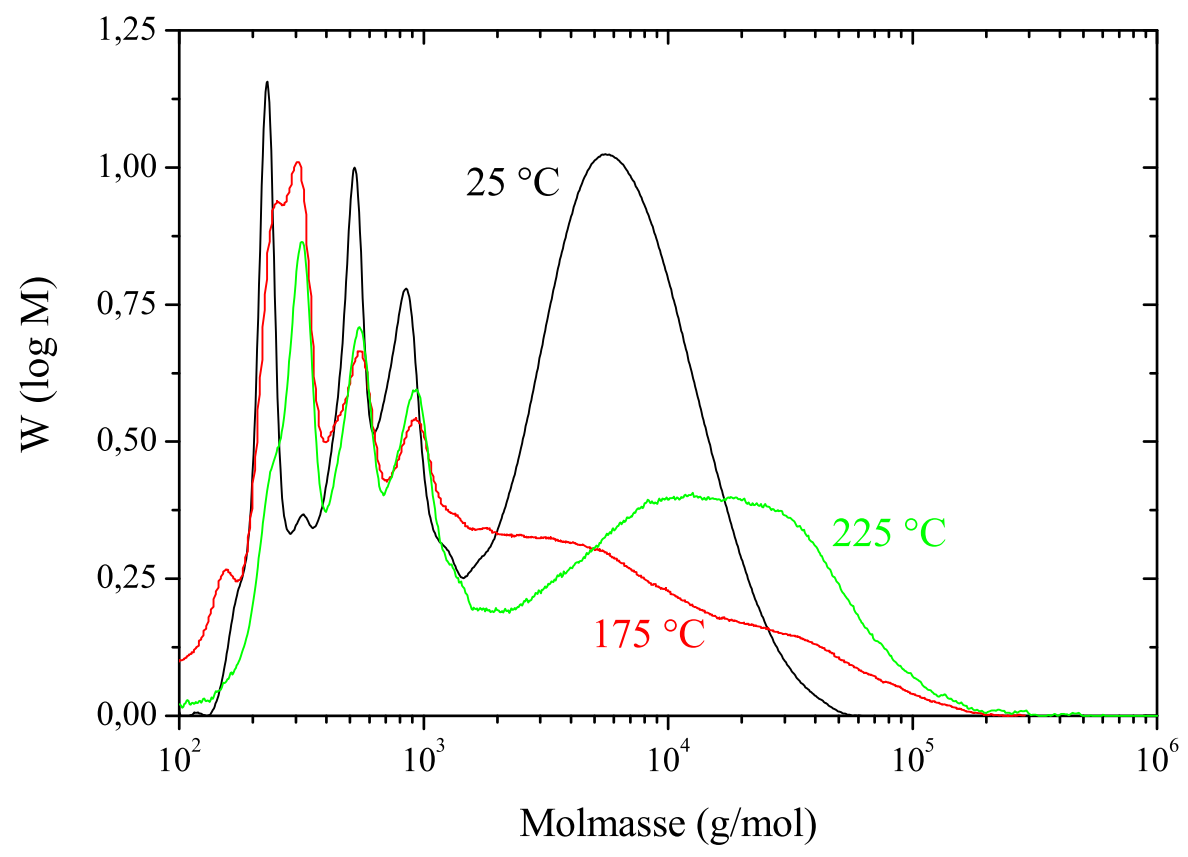

Abbildung 6.2: SE-Chromatogramme von PMMA-Filmen, die bei unterschiedlichen Substrattemperaturen hergestellt wurden (20000 Pulse, $\left.1500 \mathrm{~mJ} / \mathrm{cm}^{2}\right)$ ). 
Raumtemperatur-Probe weist - wie schon im Kapitel 4.2 beschrieben - neben Molekülen mit Molmassen von 177, 228, 318, 522, $843 \mathrm{~g} / \mathrm{mol}$, die auf die Deposition von Oligomeren zurückzuführen sind, im Wesentlichen Makromoleküle mit einer mittleren Molmasse $\mathrm{M}_{W}$ von $8300 \mathrm{~g} / \mathrm{mol}\left(\mathrm{M}_{n}=5600 \mathrm{~g} / \mathrm{mol}\right)$ auf. Bei Erhöhung der Substrattemperatur sinkt die Konzentration dieser Moleküle zu Gunsten höhermolekularen Materials ab, zu erkennen an der Schulter bei etwa $30 \mathrm{~kg} / \mathrm{mol}$ im Fall eines bei einer Substrattemperatur von $175^{\circ} \mathrm{C}$ deponierten Filmes. Mit zunehmender Substrattemperatur setzt sich dieser Trend fort. Damit verbunden steigt die mittlere Molmasse der Filme an. Kleine Veränderungen können auch in den niedermolekularen Bestandteilen der Filme beobachtet werden, wo die Konzentration der Moleküle mit einer Molmasse von $318 \mathrm{~g} / \mathrm{mol}$ auf Kosten der Moleküle mit einer Masse von $228 \mathrm{~g} / \mathrm{mol}$ steigt.

\subsection{Reaktionsmechanismus}

Abbildung 6.3 zeigt die Entwicklung der mittleren Molmasse $\mathrm{M}_{W}$ des höhermolekularen Polymers $(\mathrm{M}>2000 \mathrm{~g} / \mathrm{mol})$ mit zunehmender Substrattemperatur. $\mathrm{M}_{W}$ bleibt bis $125{ }^{\circ} \mathrm{C}$ konstant und steigt dann rasch an. Bei einer Deposition bei $250{ }^{\circ} \mathrm{C}$ können so Molmassen von $3,2 \cdot 10^{4} \mathrm{~g} / \mathrm{mol}$ erreicht werden. Anzeichen für eine Zunahme der Molmasse lassen sich auch aus der Literatur konstruieren; Abbildung 6.3 zeigt zusätzlich einen Vergleich der Molmassen laserdeponierter PMMA-Filme mit Daten von Blanchet [9], die PMMA-Filme beschreiben, die mit Hilfe eines Nd-YAG-Laser (266 nm, 15 ns) deponiert wurden. Das Molekulargewicht der Filme unterscheidet sich in den absoluten Werten der Molmassen deutlich von den hier beschriebenen Filmen, wohingegen der Verlauf der Molmassen mit zunehmender Substrattemperatur aber ähnlich ist: Bei niedrigen Substrattemperaturen weisen die Filme entsprechend einer durch den Ablationsprozess vorgegebenen Dropletdeposition konstante Molmassen unabhängig von der Temperatur auf. Oberhalb einer kritischen Temperatur steigen die Molekulargewichte jedoch exponentiell mit der reziproken Substrattemperatur an. Als kritische Temperatur kann für die hier beschriebenen Filme eine Temperatur von $125^{\circ} \mathrm{C}$ angegeben werden, während sich aus den Literaturdaten eine Temperatur von $75^{\circ} \mathrm{C}$ abschätzen lässt. Die Steigung oberhalb dieser Werte ist für beide Filmserien vergleichbar. Sie kann mit einem Arrheniusansatz beschrieben 


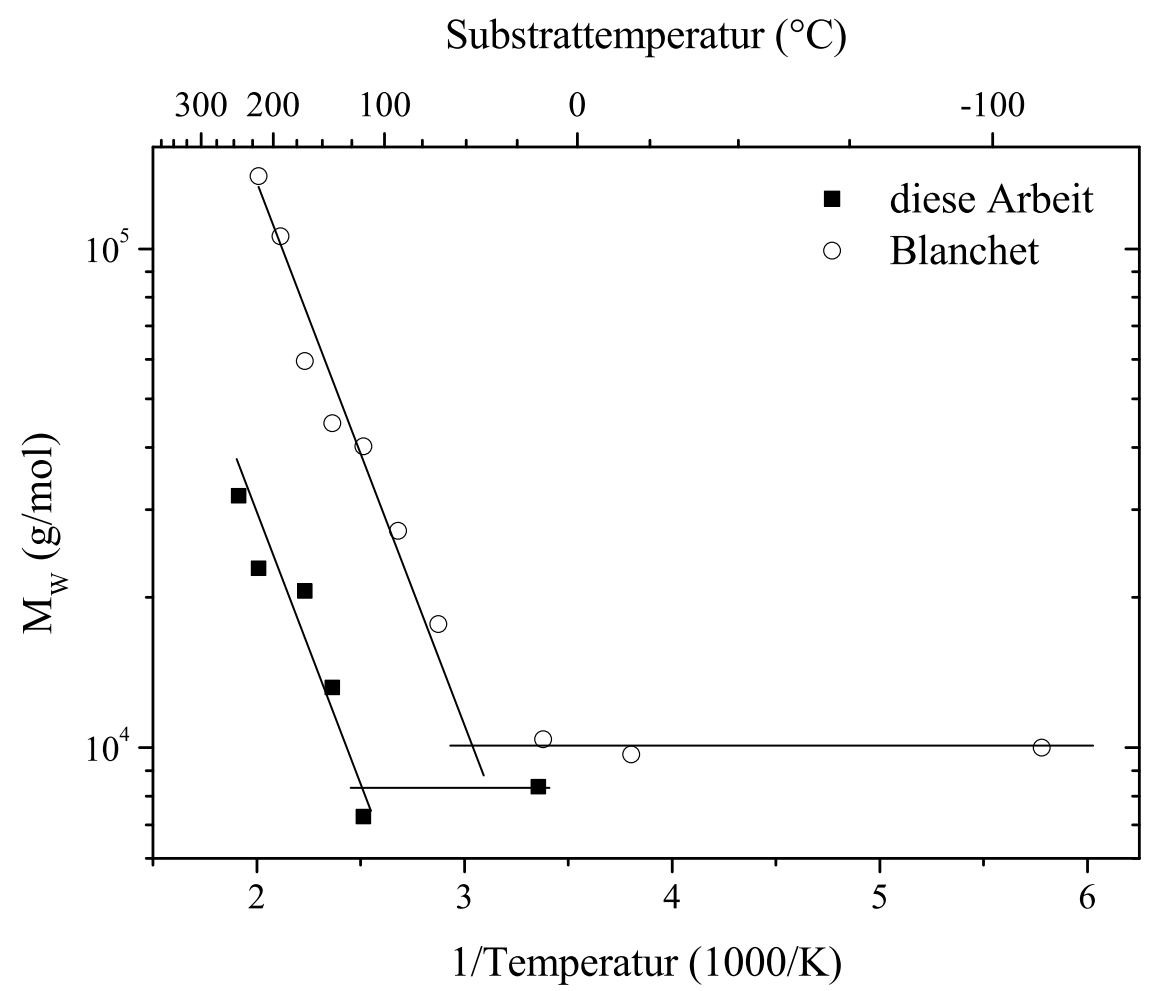

Abbildung 6.3: Mittlere Molmasse $\mathrm{M}_{W}$ der Moleküle mit einer Molmasse oberhalb von $2000 \mathrm{~g} / \mathrm{mol}$, logarithmisch gegen die reziproke Substrattemperatur aufgetragen $(\boldsymbol{\square})$. Zum Vergleich sind Daten von Blanchet [9] (○) abgebildet.

werden. Die damit abgeschätzte Aktivierungsenergie liegt bei $0,2 \mathrm{eV}$.

Der Mechanismus, der dem Zuwachs der Molmassen zu Grunde liegt, ist in beiden Fällen vergleichbar. Blanchet erklärt ihn durch eine Reaktion von Monomeren [11], die einer radikalischen Polymerisation entspricht. Obwohl die Aktivierungsenergie von 0,2 eV vergleichbar mit Aktivierungsenergien der radikalischen Polymerisation von PMMA [25] ist und ein Polymerisationsprozess auch von Tsuboi [96] vorgeschlagen wird, erscheint dieser Mechanismus auf Grund der Zusammensetzung der Ablationsprodukte aus Polymerdroplets und kleinen Degradationsprodukten fragwürdig: Die Deposition wird wesentlich durch den Übertrag von Droplets geprägt. Bei einer Deposition bei Raumtemperatur sind 60-70\% des Filmes auf die Deposition von Tröpfchen zurückzuführen (vgl. Kap. 4.2). Auch die Konstanz der 
Molmassen bei tiefen Temperaturen, die Blanchets Daten erkennbar zeigen, weist auf die Bedeutung dieser Tröpfchendeposition hin. Neben dem polymeren Anteil liegt in Filmen, die bei Raumtemperatur deponiert werden, ein Oligomer-Anteil von nur 15-20\% vor, der auf eine Reaktion von Monomeren zurückzuführen ist.

Auf Grund des geringen Anteils von Oligomeren erscheint eine radikalische Polymerisation von Monomeren als vorherrschender Prozess unwahrscheinlich. Zur detaillierteren Charakterisierung des Mechanismus lassen sich charakteristische Unterschiede der Taktizität von PMMA nutzten: Durch radikalische Polymerisation gebildetes PMMA ist ataktisch. Bei einem dominanten radikalischen Polymerisationsmechanismus sollte auch bei der Verwendung von isotaktischem Polymer als Ausgangsmaterial ein ataktisches Polymer entstehen [95].

Diese Veränderung der Taktizität lässt sich durch FTIR-Spektren von laserdeponierten Filmen eindeutig ausschließen: Abbildung 6.4 zeigt dieses exemplarisch durch einen Vergleich eines entsprechenden Spektrums eines deponierten Filmes mit dem isotaktischen Ausgangs- und einem ataktischen Referenzmaterial.

Die Unterschiede von iso- und ataktischem PMMA liegen vor allem in der Absorption der Valenzschwingungen der C-C-O-Gruppe gekoppelt mit der C-O-Gruppe im Wellenzahlenbereich von 1270-1240 $\mathrm{cm}^{-1}$ : Während isotaktisches PMMA bei $1260 \mathrm{~cm}^{-1}$ eine höhere Absorption als bei $1240 \mathrm{~cm}^{-1}$ aufweist, ist dieses Verhältnis bei ataktischem PMMA umgekehrt [20]. Vergleicht man das Spektrum des deponierten Filmes mit den Spektren von iso- und ataktischem PMMA, weist der Film deutlich charakteristische Züge von isotaktischem PMMA mit einer hohen Absorption bei $1260 \mathrm{~cm}^{-1}$ auf: Lediglich kleinere Veränderungen in den Spektren deuten - im Einklang mit Veränderungen von Oligomerkonzentrationen im SE-Chromatogramm - Reaktionen zwischen Monomeren an, die Taktizität der Filme entspricht aber wegen der Dropletdeposition im Wesentlichen dem Ausgangsmaterial.

Mit der Substrattemperatur wächst auch die Molmasse des Polymeranteils laserdeponierter PMMA-Schichten. Als Mechanismus kann ein Reaktionsmechanismus, der eine radikalische Polymerisation von Monomeren beinhaltet, als vorherrschender Prozess ausgeschlossen werden; vielmehr erscheint auf Grund der Ablationsprodukte eine Reaktion von Makromolekülen unter Bildung eines Polymers erhöhter molekularer Masse wahrscheinlich. Diese Vermutung kann mit der Deposition von PMMA mit einer genau bekannten, kleinen Molmasse $(<8000 \mathrm{~g} / \mathrm{mol}$ mit enger Molmassen- 


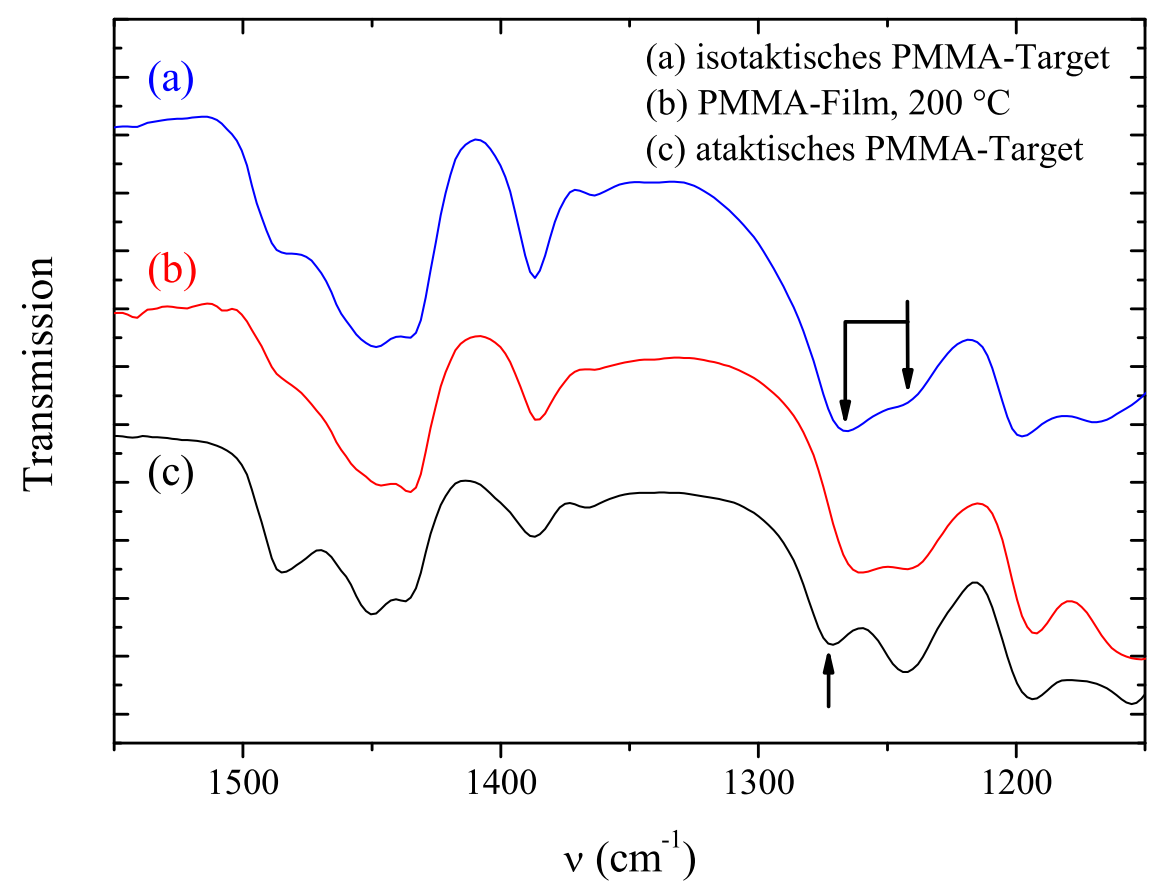

Abbildung 6.4: FTIR-Spektrum eines PMMA-Filmes nach der Deposition (2000 Pulse, $1500 \mathrm{~mJ} / \mathrm{cm}^{2}$ ) unter Verwendung eines isotaktischen PMMATargets bei einer Substrattemperatur von $200{ }^{\circ} \mathrm{C}$ (b). Zum Vergleich wurden auch Spektren von isotaktischem (a) und ataktischem PMMA (c).

verteilung) überprüft werden: Durch den Übertrag der kompletten Makromoleküle (vgl. Abb. 4.8) in Droplets stehen auf dem Substrat Produkte mit genau definierter Molmasse - entsprechend einer scharfen Molmassenverteilung - zur Verfügung. Bei einer Reaktion dieser Makromoleküle sollten Moleküle entstehen, die - ähnlich der Oligomere - ganzzahlige Vielfache dieser Molmasse aufweisen.

\section{4 Änderung der chemischen Zusammensetzung}

Neben diesen Reaktionen innerhalb der Droplets werden auch thermisch aktivierte Prozesse zwischen den beiden Ablationskomponenten angeregt, durch die die charakteristischen Unterschiede von polymerer und vernetzter Komponente auf dem Sub- 
strat verwischen. Einen ersten Hinweis auf diese Reaktionen ist in der Mikrostruktur der Filme (vgl. Abb. 6.1) zu erkennen, die bei Temperaturen zwischen der Glasübergangstemperatur und $250{ }^{\circ} \mathrm{C}$ deponiert wurden: Die Oberflächenstruktur beinhaltet Inseln, obwohl weit oberhalb der Glastemperatur eine vollständige Glättung des Filmes erwartet werden kann. Diese Inseln deuten auf eine Teilvernetzung der Tröpfchen durch Reaktionen mit kleinen Degradationsprodukten während der Deposition auf dem Substrat hin.

Chemische Reaktionen zwischen polymerem Anteil und kleinen Degradations-

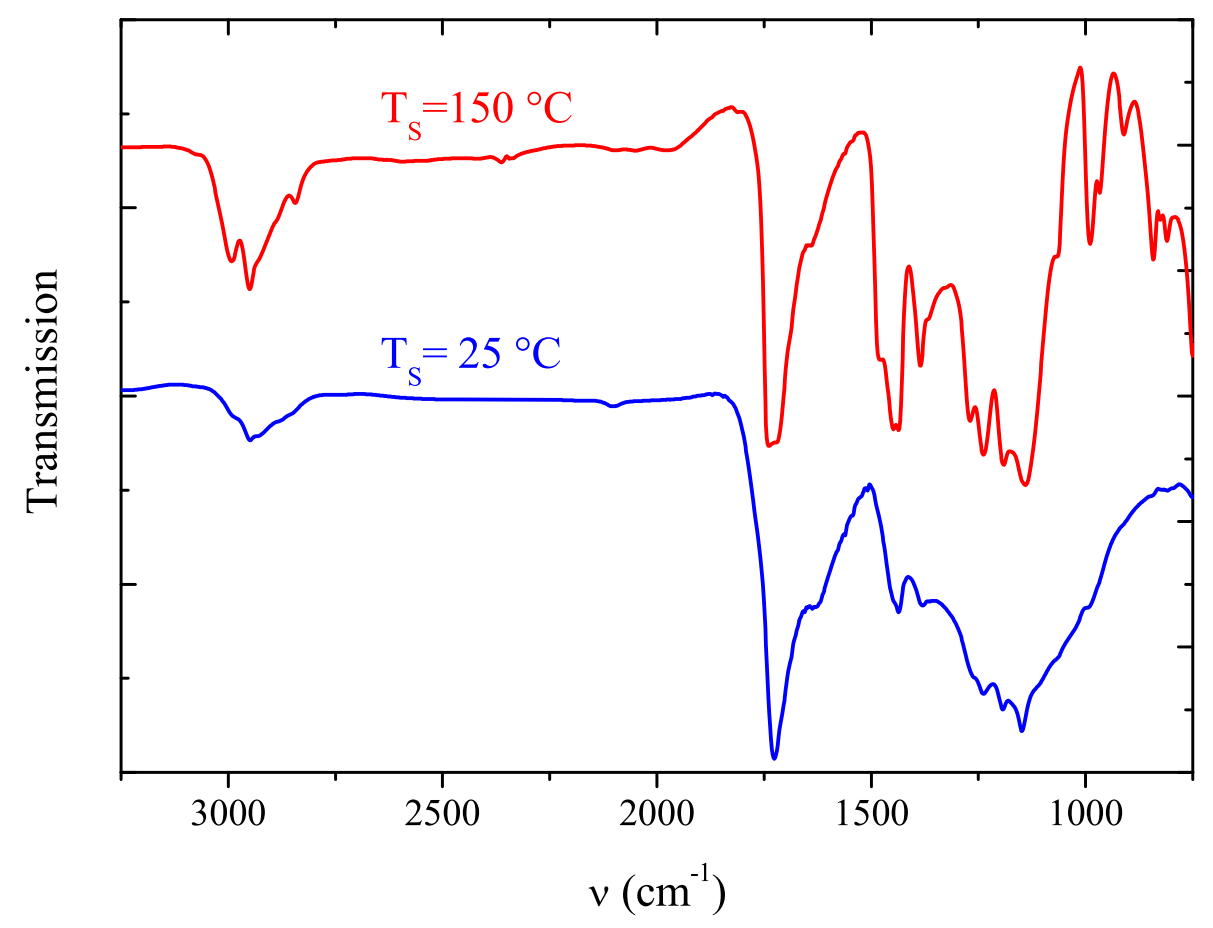

Abbildung 6.5: IR-Spektrum des unlöslichen Rückstandes von Filmen, die bei unterschiedlichen Substrattemperaturen hergestellt wurden. Beide Proben wurden eine Stunde mit THF behandelt und nach der Entfernung des Lösungsmittels inklusive gelöster Polymerbestandteile für 48 Stunden im Vakuum $\left(<10^{-7}\right.$ mbar $)$ ausgelagert, um Lösungsmittelrückstände im Spektrum $\mathrm{zu}$ vermeiden. 
produkten führen zu einer Vermischung der deponierten Komponenten, indem in der vernetzten Komponente auch hochmolekulares Polymer gebunden wird. Abbildung 6.5 belegt diese Vermischung am Beispiel eines IR-Spektrums des in organischen Lösungsmitteln wie THF unlöslichen Rückstandes eines laserdeponierten PMMAFilmes. Das Spektrum dieses vernetzten Filmanteils weist PMMA-typische Banden auf, die bei vergleichbaren, bei Raumtemperatur deponierten Filmen auf Grund des Vernetzungsprozesses nicht nachweisbar sind (vgl. Kap. 4.4). Am Beispiel der Streckschwingung der CH-Gruppen bei $2950 \mathrm{~cm}^{-1}$, und der Deformationsschwingungen der $\mathrm{CH}_{2}$-Gruppe $\left(1484 \mathrm{~cm}^{-1}\right)$, der $\mathrm{CH}_{3}$-Gruppe $\left(1385 \mathrm{~cm}^{-1}\right)$, oder der $\mathrm{OCH}_{3}$-Gruppe $\left(989 \mathrm{~cm}^{-1}\right)$ wird dies besonders deutlich.

\subsection{Thermische Zersetzung der Filme}

Die chemische Zusammensetzung laserdeponierter PMMA-Filme reflektiert bei Substrattemperaturen bis zu $200{ }^{\circ} \mathrm{C}$ die Veränderungen der polymeren Komponente mit einem Anstieg der Molmasse und vernetzter Schicht, in die hochmolekulares PMMA eingebaut wird. Oberhalb dieser Temperaturen kann eine thermische Degradation von PMMA einsetzen [48]. Als zusätzlicher Prozess kann dieser Zerfall des deponierten Polymers, die durch eine Abspaltung der Carbonylgruppe [68] initiiert wird, am Absorptionsrückgang der entsprechenden Bande bei $1730 \mathrm{~cm}^{-1}$ in IR-Spektren beobachtet werden. In Abbildung 6.6 ist dies exemplarisch durch ein IR-Spektrum eines bei einer Substrattemperatur von $300{ }^{\circ} \mathrm{C}$ laserdeponierten PMMA-Filmes belegt, der mit dem Spektrum eines bei Raumtemperatur deponierten Filmes verglichen wird: Vor allem im Wellenzahlenbereich von $1800-750 \mathrm{~cm}^{-1}$ zeigt die bei $300{ }^{\circ} \mathrm{C}$ deponierte Schicht eine vergleichsweise schwache Absorption. Abbildung 6.7 zeigt die thermische Degradation anhand der Absorption der Carbonylbande, die auf die Intensität der C-H-Streckschwingungen (bei $2950 \mathrm{~cm}^{-1}$ ) bezogen wurde. Das Verhältnis dieser Schwingungen zeigt bis zu Substrattemperaturen von etwa $175^{\circ} \mathrm{C}$ keine signifikanten Veränderungen, fällt aber bei weiterer Erhöhung der Temperatur auf $60 \%$ des Ausgangswertes ab. Im Vergleich zur Degradation von deponierten PMMA-Schichten ist diese Zerfallstemperatur um mehr als $20 \mathrm{~K}$ verschoben [91] (vgl. Kap. 7.2). Diese Verschiebung kann auf bei der Deposition in den Droplets gespeicherte Energie zurückzuführen sein. Die Degradation von PMMA führt zu Monomeren und klei- 


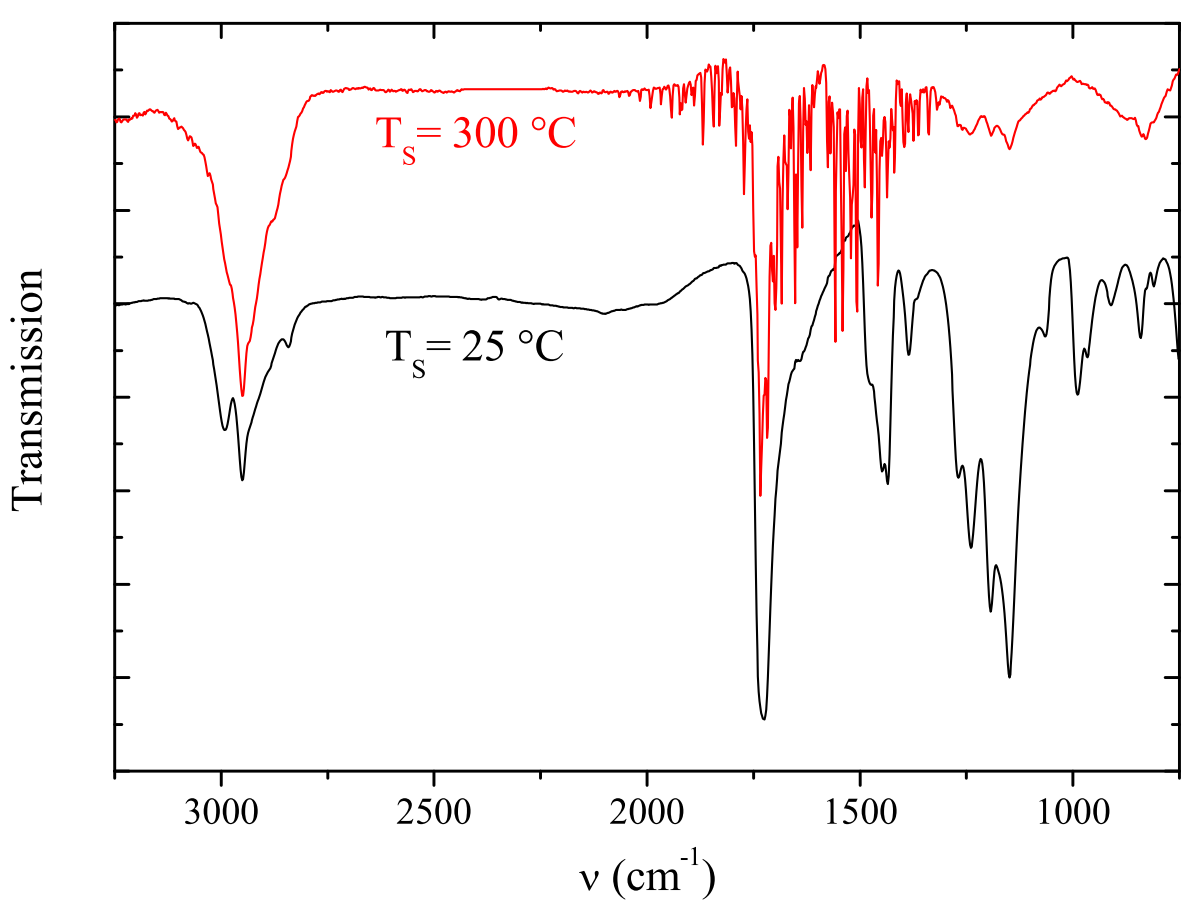

Abbildung 6.6: FTIR-Spektrum eines PMMA-Filmes, der auf ein auf $300{ }^{\circ} \mathrm{C}$ geheiztes Substrat deponiert wurde. Zum Vergleich ist das Spektrum eines bei Raumtemperatur deponierten Filmes mit abgebildet.

nen thermischen Zerfallsprodukten, die im Wesentlichen den Ablationsprodukten (vgl. Kap. 3.1) entsprechen. Daher erscheint es wahrscheinlich, dass diese Produkte in einem Prozess ähnlich der Deposition kleiner UV-Degradationsprodukte (vgl. Kap. 4.4) auf dem Substrat gebunden werden. Ein getrennter experimenteller Nachweis der Reaktionsprodukte des thermischen Zerfalls gestaltet sich auf Grund dieser Analogie als schwierig.

Grundsätzlich kann die Degradation von PMMA als konkurrierender Prozess zur Zunahme des Molekulargewichts bei hohen Temperaturen angesehen werden; sie begrenzt damit das maximale Molekulargewicht. Bis zu Substrattemperaturen von $250{ }^{\circ} \mathrm{C}$ wird aber kein Abknicken der Molmassen (vgl. Abb. 6.3) beobachtet, so dass als obere Schranke eine Substrattemperatur oberhalb von $250{ }^{\circ} \mathrm{C}$ angegeben werden kann. 


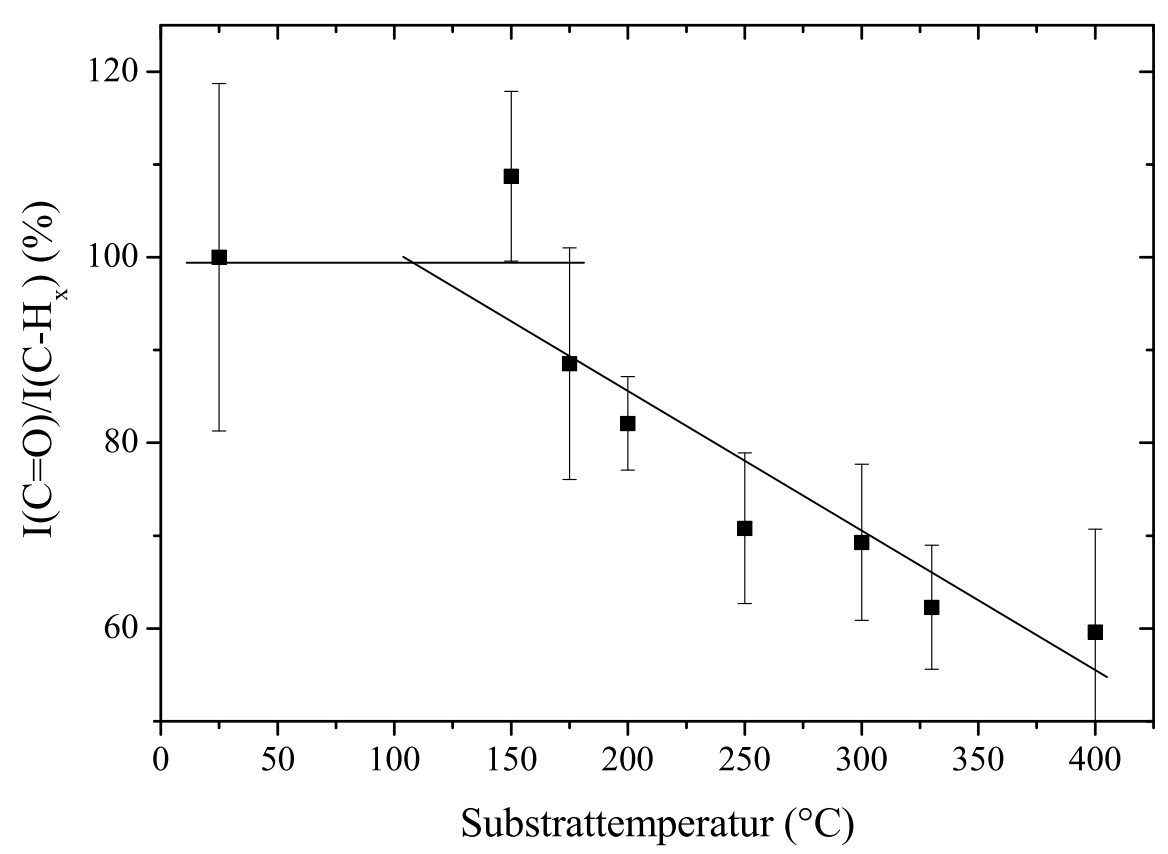

Abbildung 6.7: Absorptionsverhältnis von Carbonyl- und C-HStreckschwingungen, gegen die reziproke Substrattemperatur aufgetragen. Zur Vermeidung von Abweichungen durch nicht identische Depositionsbedingungen (Energiedichte) wurden gleichzeitig Filme auf einem Substrat bei Raumtemperatur hergestellt und die Absorptionsverhältnisse als relative Abweichungen von den Absorptionsverhältnissen bei Raumtemperatur dargestellt.

\subsection{Mechanische Eigenschaften bei unterschiedlichen Substrattemperaturen}

Durch eine Deposition auf einem geheizten Substrat kann die Zusammensetzung der Filme eingestellt werden: Thermisch aktivierte Prozesse führen zu einer Zunahme der Molmasse des Polymers. Gleichzeitig wird auch die vernetzte Komponente der Schicht durch den Einbau von hochmolekularem PMMA verändert. Bei hohen Temperaturen kann die Zusammensetzung der Filme zusätzlich durch eine Degradation des deponierten Materials auf dem Substrat modifiziert werden. 
Die makroskopischen mechanischen Eigenschaften der Filme, die - wie im Kapitel 4.5 dargestellt - wesentlich durch die Koexistenz von unvernetztem Polymer reduzierter Molmasse neben einer vernetzten Komponente bestimmt werden, repräsentieren diese Veränderungen der chemischen Zusammensetzung: Die Filme weisen wegen der Zunahme der Molmasse eine Verfestigung auf, die in Abbildung 6.8 am Beispiel des reduzierten Elastizitätsmodul $E_{\mathrm{HU}} /\left(1-\nu^{2}\right)$ für unterschiedliche Substrattemperaturen dargestellt ist. Der Elastizitätsmodul bleibt bis zu Temperaturen von $125^{\circ} \mathrm{C}$ konstant bei 3,4 GPa und steigt dann mit der Substrattemperatur auf 7 GPa bei $250^{\circ} \mathrm{C}$ an. Die Verfestigung der Filme zeigt ein qualitativ ähnliches Bild wie der Anstieg der Molmasse (vgl. Abb. 6.3) mit einem konstanten Plateau bis zu einer kritischen Temperatur von $125^{\circ} \mathrm{C}$ und dann einem exponentiellen Anstieg mit der reziproken Temperatur. Auch die abgeschätzte Aktivierungsenergie liegt mit $0,15 \mathrm{eV}$ in der gleichen Größenordnung.

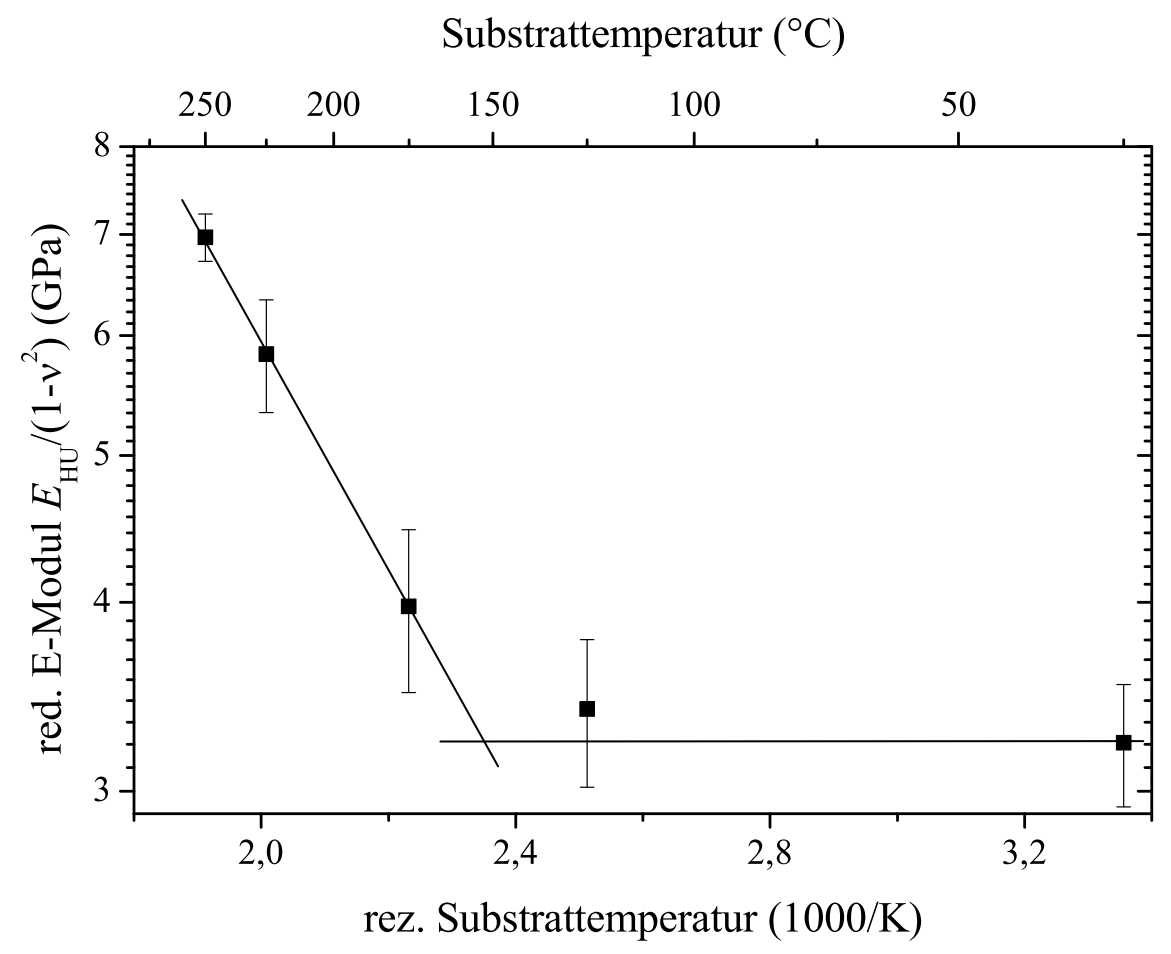

Abbildung 6.8: Reduzierter E-Modul $E_{\mathrm{HU}} /\left(1-\nu^{2}\right)$ von PMMA-Filmen (1500 mJ $/ \mathrm{cm}^{2}, 10000$ Pulse) als Funktion der Substrattemperatur. 
Die Verfestigung der Filme ist aber nicht allein auf die Zunahme der Molmasse zurückzuführen. Vielmehr steigen die Elastizitätsmodulen stärker an als die Molmasse: Abbildung 6.9 zeigt einen Vergleich der reduzierten elastischen Modulen von laserdeponierten PMMA-Proben mit unterschiedlichen mittleren Molmassen $\mathrm{M}_{W}$, bestimmt durch SE-Chromatographie, mit den Modulen von PMMA-Folien unterschiedlicher Molekulargewichte. Der Elastizitätsmodul der PMMA-Folien steigt von 3,1 GPa bei einer Molmasse von 3,9 kg/mol bis auf $5 \mathrm{GPa}\left(\mathrm{M}_{W}=350 \mathrm{~kg} / \mathrm{mol}\right)$ an. Auch die Modulen der laserdeponierten Proben wachsen an. Bis zu einer Molmasse von etwa $20 \mathrm{~kg} / \mathrm{mol}$ ist die Zunahme mit der Änderung der elastischen Modulen der PMMA-Folien vergleichbar. Die weitere Entwicklung der Modulen der laserdeponierten Proben ist hingegen deutlich steiler.

Diese starke Verfestigung laserdeponierter PMMA-Filme kann erst bei Sub-

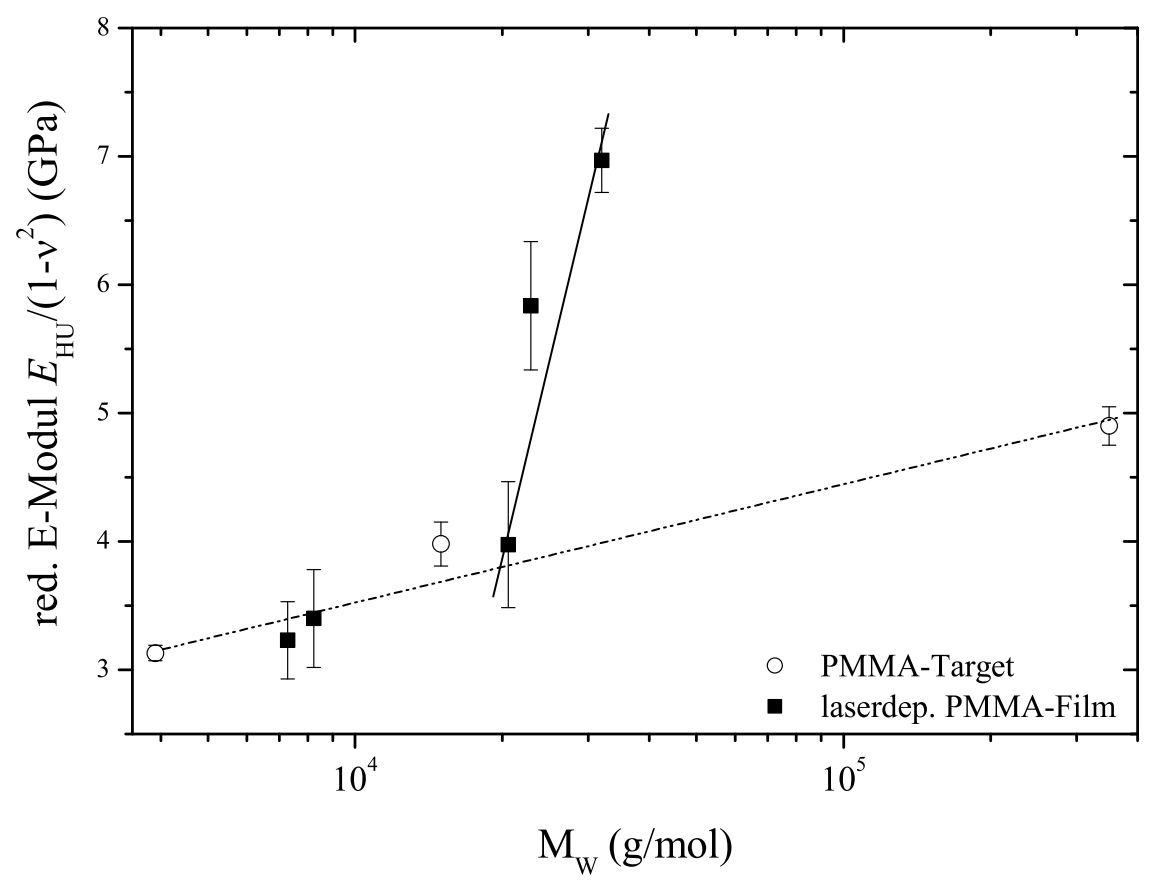

Abbildung 6.9: Reduzierter E-Modul $E_{\mathrm{HU}} /\left(1-\nu^{2}\right)$ laserdeponierter Filme (10000 Pulse, $1500 \mathrm{~mJ} / \mathrm{cm}^{2}$ ) (ם) und reduzierter E-Modul unterschiedlicher PMMA-Targets $(\bigcirc)$ in Abhängigkeit der mittleren Molmasse $\mathrm{M}_{W}$. 
strattemperaturen oberhalb von $200{ }^{\circ} \mathrm{C}$ nachgewiesen werden. Als Erklärung sind verschiedene Prozesse denkbar: Die Zusammensetzung der vernetzten Komponente wird durch den Einbau von hochmolekularem PMMA verändert. Da die mechanischen Eigenschaften auch von dieser vernetzten Schicht abhängen (vgl. Kap. 4.5), erscheint eine Veränderung wahrscheinlich. Auch Modifikationen der mechanischen Eigenschaften durch den Einfluss der thermischen Degradation können nicht ausgeschlossen werden. Mit den hier beschriebenen Experimenten können die Prozesse, die die makroskopischen mechanischen Eigenschaften bestimmen, nicht klar identifiziert werden. Zur eindeutigen Beschreibung der Verfestigungsmechanismen bieten sich weiterführende Experimente an, in denen die mechanischen Eigenschaften der unterschiedlichen Komponenten durch ortsaufgelöste AFM-Messungen beschrieben werden.

Alle Ergebnisse lassen sich verstehen, indem die Ablationssprodukte während der Deposition auf dem Substrat zusätzliche Energie erhalten. Dadurch werden thermisch aktivierte Prozesse angeregt, die die Eigenschaften der Schichten maßgeblich verändern: Bei einer Deposition oberhalb der Glastemperatur verhalten sich die deponierten Polymerdroplets wie eine viskose Flüssigkeit und die Mikrostruktur der Filme weist auf Grund von Glättungsmechanismen eine vergleichsweise niedrige Rauigkeit auf. Chemische Reaktionen der Makromoleküle untereinander erhöhen die Molmasse des Polymeranteils der Schicht. Ferner kann durch Reaktionen zwischen Makromolekülen und kleinen Degradationsprodukten hochmolekulares PMMA in die vernetzte Schicht eingebaut werden. 


\section{7}

\section{Thermische Veränderung der Filme: Relaxation und Zerfall}

In diesem Kapitel werden die Veränderungen laserdeponierter PMMA-Filme und ihrer Eigenschaften bei einer thermischen Auslagerung untersucht.

\subsection{Glasübergang laserdeponierter Filme}

Die Eigenschaften laserdeponierter PMMA-Filme werden wesentlich vom Polymeranteil der Filme geprägt. Abbildung 7.1 zeigt einen Vergleich eines dielektrischen Verlustspektrums eines laserdeponierten PMMA-Films (a) mit dem entsprechenden Spektrum einer PMMA-Folie (b), aufgenommen gegen die Temperatur bei einer konstanten Frequenz von $5,4 \mathrm{kHz}$ in Zusammenarbeit mit dem I. Physikalischen Institut der Universität Göttingen und dem Lehrstuhl für Experimentalphysik V der Universität Augsburg. Der dielektrische Verlust beider Proben steigt zunächst mit zunehmender Temperatur an. Das Maximum des Verlustes der laserdeponierten Probe wird bei etwa $100^{\circ} \mathrm{C}$ erreicht. Es ist auf die mit dem kalorischen Glasübergang verbundene $\alpha$-Relaxation und die $\beta$-Relaxation der polymeren Komponente zurückzuführen, bei der die $\mathrm{C}(\mathrm{C}=\mathrm{O}) \mathrm{OCH}_{3}$-Gruppe durch Torsion Energie aufnimmt. Diese Relaxationen, die bei einer Anregungsfrequenz von 5,4 kHz zusammenfallen (merging, [1]) können auch in der PMMA-Folie nachgewiesen werden, bei der die Temperatur der maximalen Dämpfung aber mit $120{ }^{\circ} \mathrm{C}$ deutlich höher liegt. Da die $\alpha$-Relaxation von der Molmasse des Polymers abhängt, ist diese Verschiebung auf 


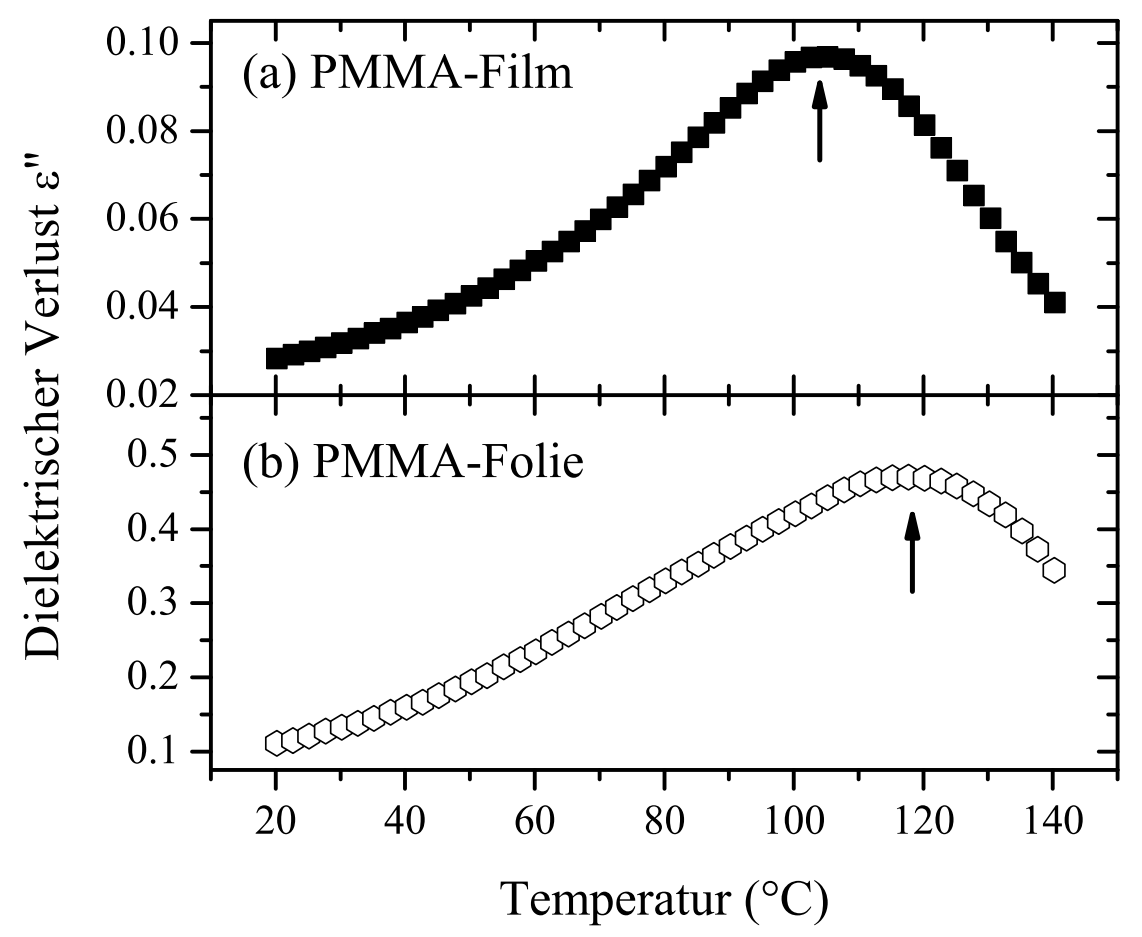

Abbildung 7.1: Dielektrische Verlustspektren eines laserdeponierten PMMAFilmes (1500 mJ $/ \mathrm{cm}^{2}, 5000$ Pulse) (a) und einer PMMA-Folie (b), aufgenommen bei konstanter Frequenz von 5,4 kHz [76].

die reduzierte Molmasse der laserdeponierten PMMA-Filme zurückzuführen. Mit Hilfe der von O'Driscoll und Amin Sanayei [73] gefundenen empirischen Beziehung

$$
T_{g}=T_{g, \infty}-K / X_{n}^{2 / 3}
$$

lässt sich die Glasübergangstemperatur $T_{g}$ des deponierten Polymers aus der Anzahl der durchschnittlichen Monomereinheiten $X_{n}$ eines Makromoleküls abschätzen. Die dabei verwendeten Konstanten sind materialspezifisch, $T_{g, \infty}$ beschreibt die Glastemperatur eines PMMA-Makromoleküls mit unendlicher Kettenlänge. Unter der Annahme einer Molmasse $\mathrm{M}_{n}$ von $5600 \mathrm{~g} / \mathrm{mol}$ erhält man so eine Übergangstemperatur von etwa $90{ }^{\circ} \mathrm{C}$, und damit eine Verschiebung der Glastemperatur um etwa 20 K im Vergleich zum Ausgangsmaterial. Diese Verschiebung liegt im gleichen Rah- 
men wie die experimentell beobachtete Verschiebung des maximalen dielektrischen Verlustes. Ein direktes Ablesen der Glasübergangstemperaturen ist auf Grund der Verschmelzung von $\alpha$ - und $\beta$-Relaxation bei einer Anregungsfrequenz von $5,4 \mathrm{kHz}$ nicht möglich. Daher kann aus den hier beschriebenen Experimenten auch nicht ausgeschlossen werden, dass auch die $\beta$-Relaxation von der Molmasse oder von Veränderungen der PMMA-Schichten in Folge von Depositionsprozessen beeinflusst wird; eine zusätzliche Verschiebung der Glasübergangstemperatur durch die Reduktion einer Dimension [49, 31, 32] oder den Einfluss des Substrates (z.B. [50, 99, 105]) kann auf Grund der hohen Schichtdicke aber vernachlässigt werden.

In Abbildung 7.2 wird die Zusammensetzung der Schichten bei einer Auslagerung mit Hilfe der FTIR-Spektroskopie charakterisiert. Bis zu Auslagerungstempe-

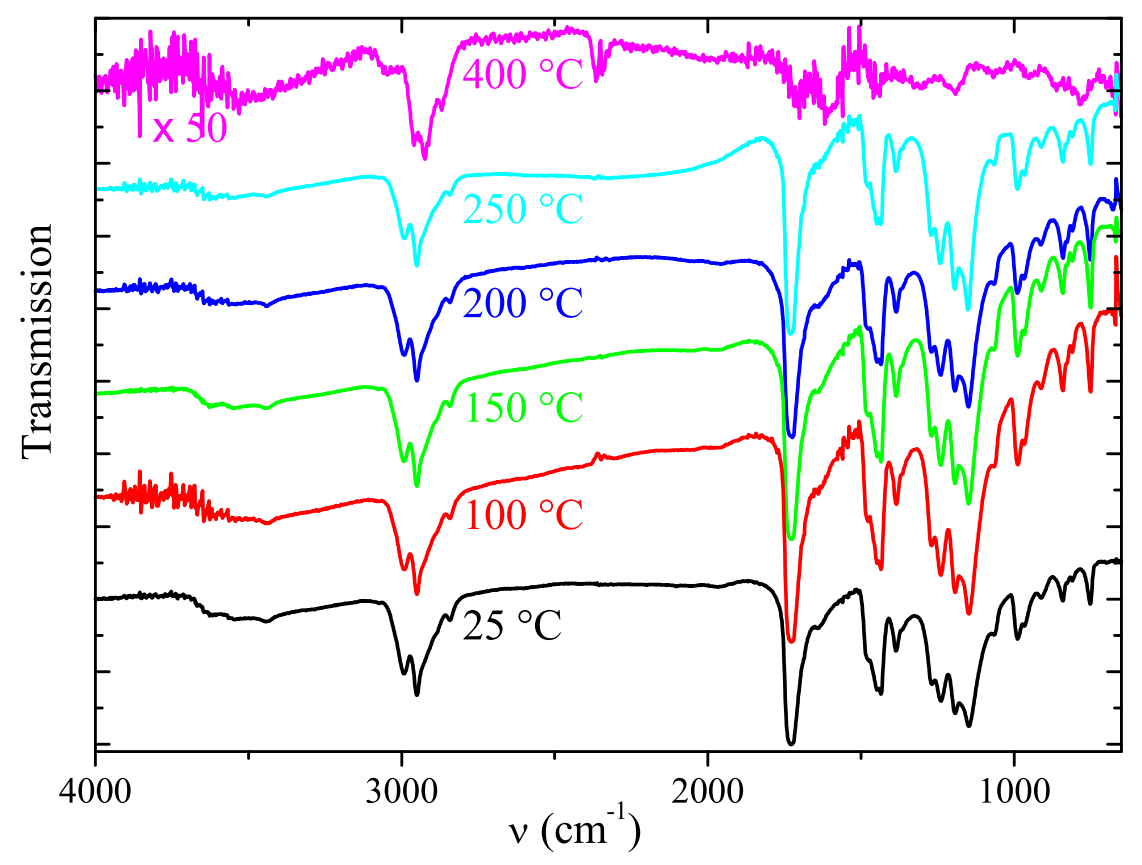

Abbildung 7.2: FTIR-Spektren von PMMA-Filmen nach der Herstellung bei Raumtemperatur, bzw. nach unterschiedlichen Auslagerungsschritten, jeweils für eine Stunde im Vakuum $\left(<10^{-7}\right.$ mbar). Die Spektren wurden zur besseren Übersicht verschoben. Das Spektrum der bis auf $400^{\circ} \mathrm{C}$ ausgelagerten Schicht wurde zusätzlich mit einem Faktor 50 multipliziert. 
raturen von etwa $250{ }^{\circ} \mathrm{C}$ zeigen die Filme ähnliche Spektren. Alle Banden sind im Vergleich zum Spektrum eines einfachen, nicht ausgelagerten Filmes vorhanden und weisen keine bemerkenswerten Absorptionsveränderungen auf. Die Zusammensetzung der Filme ändert sich in diesem Temperaturbereich also nicht signifikant. Auch die Molmasse der deponierten Filme ist keiner größeren Veränderung unterworfen. Abbildung 7.3 zeigt die SE-Chromatogramme von Proben, die auf unterschiedliche Temperaturen ausgelagert wurden. Die nachgewiesenen Droplet-Molmassen sind in allen drei Chromatogrammen vergleichbar, kleine Veränderungen können lediglich in der Konzentration der Moleküle verzeichnet werden. Bei einer Auslagerung verhalten sich beide Komponenten daher bis zu $200{ }^{\circ} \mathrm{C}$ stabil und chemische Reaktionen treten in den Filmen nicht auf.

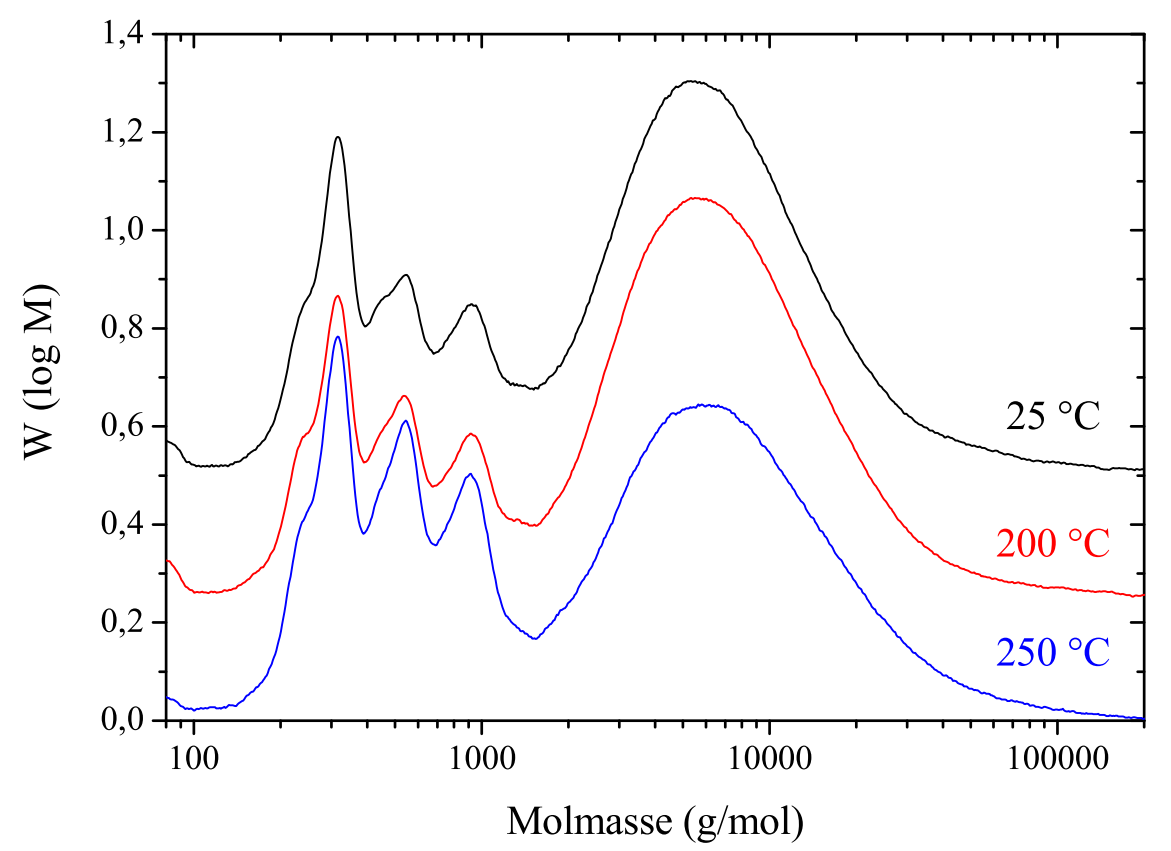

Abbildung 7.3: Molmassenverteilung laserdeponierten PMMA-Filme (1500 mJ $/ \mathrm{cm}^{2}, 10000$ Pulse) nach der Auslagerung. Als Eluent wurde THF verwendet. 


\subsection{Zerfall des Polymers}

Abbildung 7.4 zeigt eine thermogravimetrische Analyse (TGA) eines laserdeponierten Filmes. Im Einklang mit der FTIR-Spektroskopie zeigt die TGA bei Auslagerungen bis zu $215^{\circ} \mathrm{C}$ keine signifikanten Massenveränderungen. Bei höheren Auslagerungstemperaturen verliert die Probe jedoch stark an Gewicht und der polymere Anteil der Filme zerfällt in einem Temperaturbereich bis $400{ }^{\circ} \mathrm{C}$.

Diese thermische Degradation ist typisch für PMMA [48, 68]. Entsprechend zeigt die thermogravimetrische Analyse einer PMMA-Folie einen ähnlichen Verlauf (vgl. Abb. 7.4 (b)), wobei sich lediglich die Zerfallstemperaturen um etwa $35 \mathrm{~K}$ un-

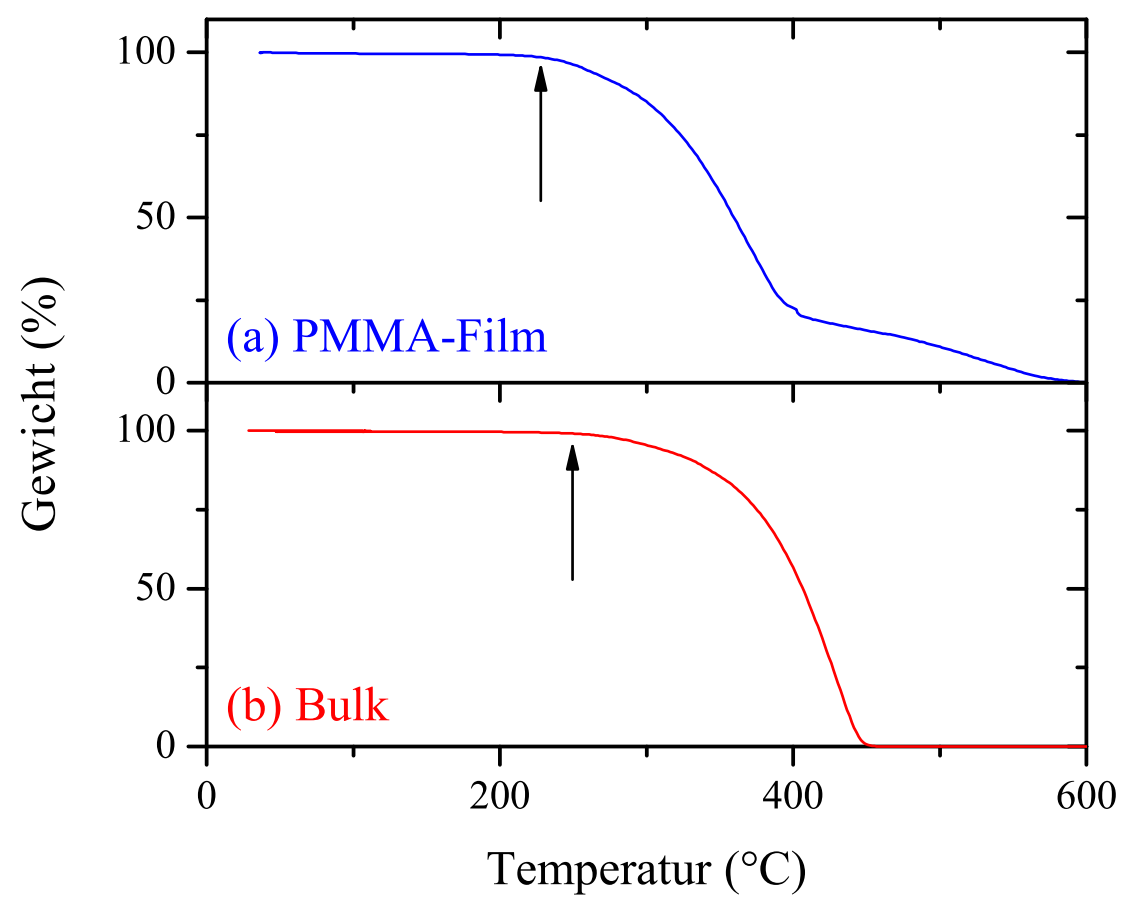

Abbildung 7.4: Thermogravimetrische Analyse eines PMMA-Films (a) (2000 mJ $/ \mathrm{cm}^{2}$, 5000 Pulse, Substrat $\mathrm{Al}$ ), aufgenommen mit einer Heizrate von $20 \mathrm{~K} / \mathrm{min}$ in Ar-Atmosphäre. Zum Vergleich ist auch eine TGA eines PMMA-Targets abgebildet (b). 
terscheiden. Die niedrigere Zerfallstemperatur des laserdeponierten Filmes ist auf die unterschiedlichen Molmassen der Polymere zurückzuführen: Mit der Reduktion der Molmasse von $335 \mathrm{~kg} / \mathrm{mol}$ im Ausgangsmaterial auf 8,3 kg/mol im laserdeponierten Film ist eine Absenkung der Aktivierungsenergie der thermischen Degradation verbunden [48].

Die Degradation von PMMA wird über eine Abspaltung der Carbonylgruppe initiiert [68] (vgl. Abb. 3.2). In den IR-Spektren (vgl. Abb. 7.2) äußert sich der thermische Zerfall des Polymers vor allem durch einen Absorptionsverlust dieser Schwingung bei $1730 \mathrm{~cm}^{-1}$. Der weitere Zerfall wird im Wesentlichen durch eine Depolymerisation unter Bildung des Monomeren Methylmethacrylat realisiert, das bei einer Auslagerung auf Grund der hohen Flüchtigkeit (Dampfdruck von 42,7 mbar

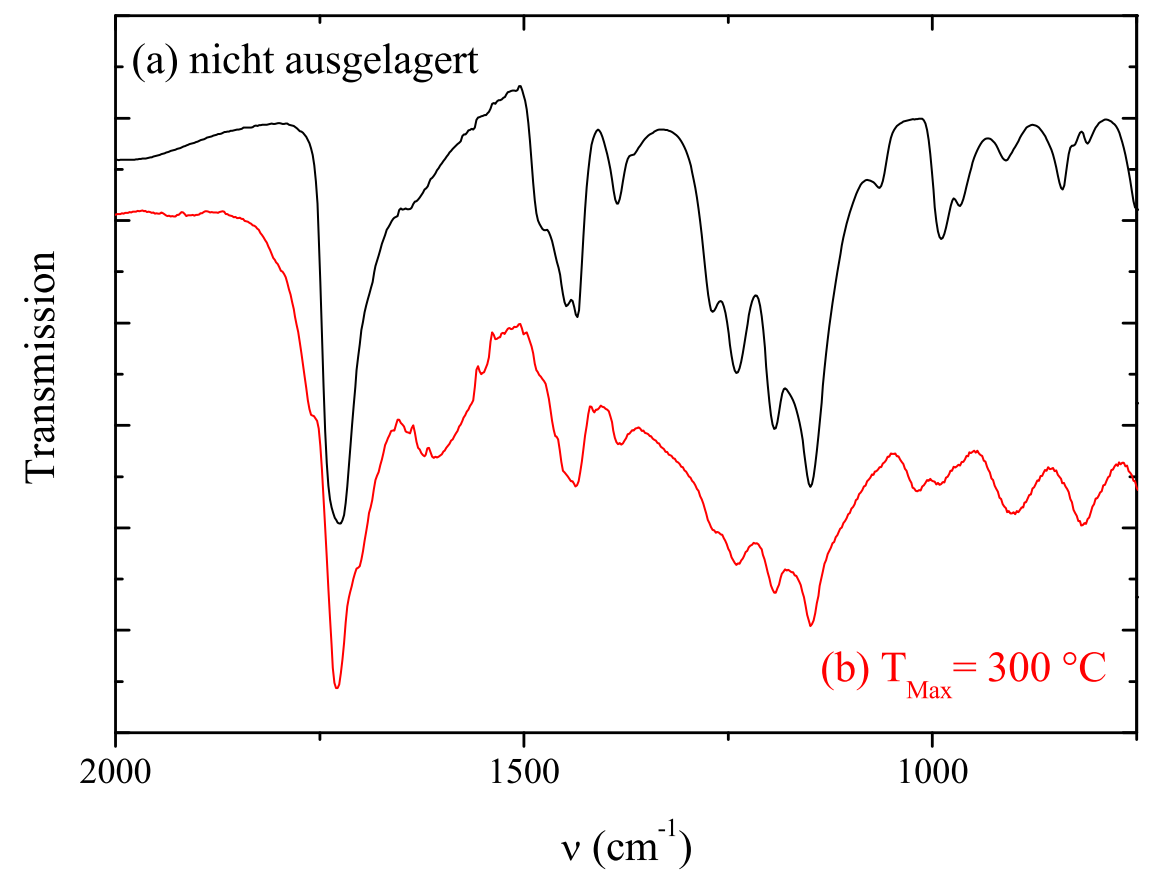

Abbildung 7.5: FTIR-Spektrum einer bis auf $300{ }^{\circ} \mathrm{C}$ ausgelagerten PMMAProbe (b), (1500 mJ $/ \mathrm{cm}^{2}, 5000$ Pulse). Zum Vergleich ist auch das Spektrum einer unbehandelten, bei Raumtemperatur unter gleichen Bedingungen hergestellten Schicht dargestellt (a). 
bei $20{ }^{\circ} \mathrm{C}$ [30]) aus dem Film entfernt wird. Dieser Materialverlust wird vor allem durch einen Absorptionsrückgang im Fingerprintbereich (750-1500 cm${ }^{-1}$ ) sichtbar, andere Banden wie die C-H-Streckschwingungen bei $3000 \mathrm{~cm}^{-1}$ werden teilweise verschoben. Abbildung 7.5 zeigt einen Ausschnitt eines IR-Spektrums, das neben einem Absorptionsrückgang im Fingerprintbereich eine neue Bande bei $1610 \mathrm{~cm}^{-1}$ aufweist. Diese Bande deutet eine Veränderung der Bindungsverhältnisse in der Nebengruppe $\left(-\mathrm{C}(=\mathrm{O}) \mathrm{OCH}_{3}\right)$ an [94]. Auch die Bildung einer $\mathrm{C}=\mathrm{C}-$ Doppelbindung, die als Bande bei $1642 \mathrm{~cm}^{-1}$ [58] auftritt, ist wahrscheinlich.

Detaillierter lässt sich die thermische Degradation bei unterschiedlichen Auslagerungstemperaturen durch die Entwicklung der Carbonylbande beschreiben. In Abbildung 7.6 wird die Absorption dieser Schwingung (bei $1730 \mathrm{~cm}^{-1}$ ) mit der der C-H-Streckschwingungen bei $2950 \mathrm{~cm}^{-1}$ verglichen. Das Verhältnis dieser Banden

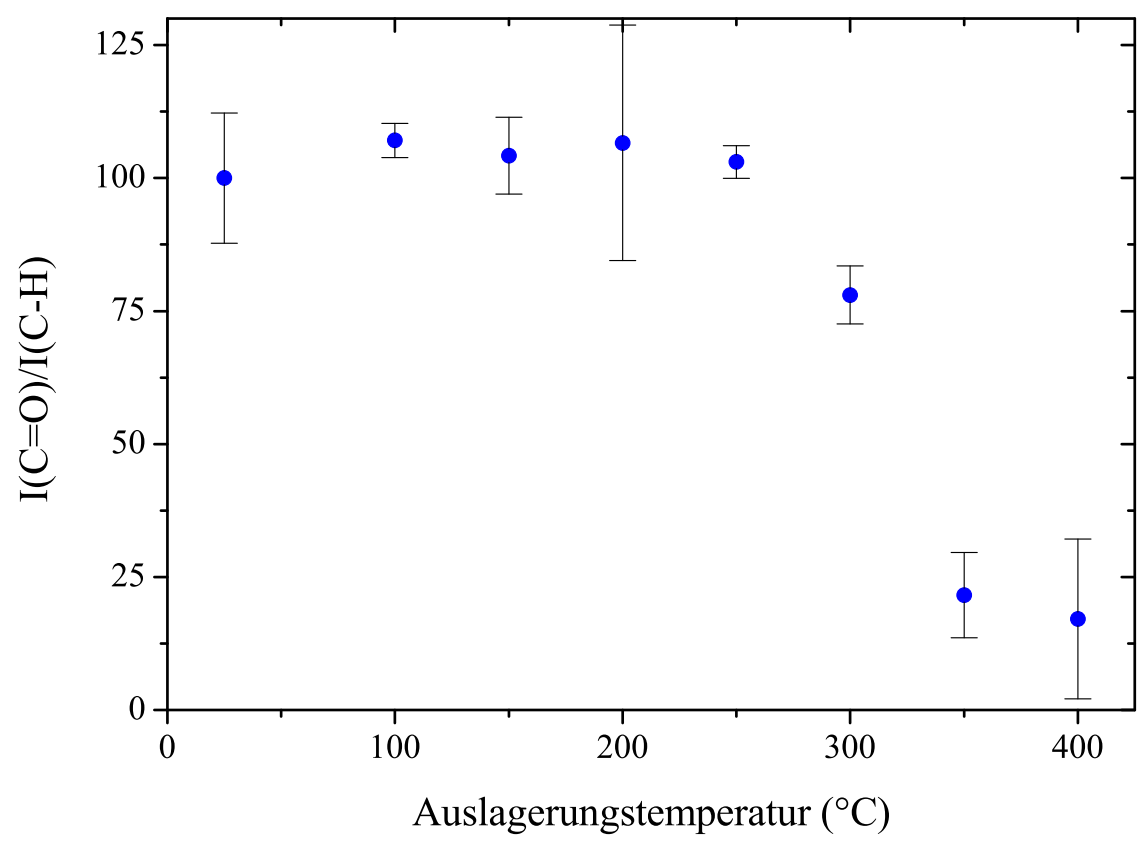

Abbildung 7.6: Verhältnis der IR-Intensitäten der Carbonyl- und Methylgruppe für unterschiedliche Auslagerungstemperaturen (Abb. 7.2), normiert auf das Verhältnis des entsprechenden Filmes vor der Auslagerung. 
weist bis zu Auslagerungstemperaturen von $250{ }^{\circ} \mathrm{C}$ keine signifikanten Veränderungen auf. Bei höheren Temperaturen nimmt die Absorption der Banden in den FTIR-Spektren jedoch ab. Diese Abnahme ist - bis auf den Beginn des Zerfalls bei Temperaturen oberhalb von $250{ }^{\circ} \mathrm{C}$ - vergleichbar mit dem in der TGA beobachteten Massenverlust. Die Differenz der Zerfallstemperaturen begründet sich durch die Insensibilität der IR-Spektroskopie auf kleine Massenänderungen ohne große Veränderung der chemischen Zusammensetzung.

\subsection{Vernetzte Komponente als Rückstand}

Bei $400{ }^{\circ} \mathrm{C}$ ist der Zerfall des Polymers abgeschlossen. Übrig bleibt ein Rückstand, der etwa 20 Gew.-\% des Ausgangsmaterials ausmacht (vgl. Abb. 7.4). Dieser Rückstand ist auf die vernetzte Komponente des laserdeponierten Filmes zurückzuführen. Abbildung 7.7 zeigt die Mikrostruktur dieses Rückstandes anhand eines Vergleiches einer elektronenmikroskopischen Aufnahme eines bis auf $400{ }^{\circ} \mathrm{C}$ ausgelagerten PMMA-Filmes mit der Aufnahme eines entsprechenden Filmes vor der Auslagerung. Beide Aufnahmen zeigen eine ähnliche Mikrostruktur, die von deponierten Droplets geprägt wird. Da diese Droplets bereits bei der Deposition durch ein Überwachsen

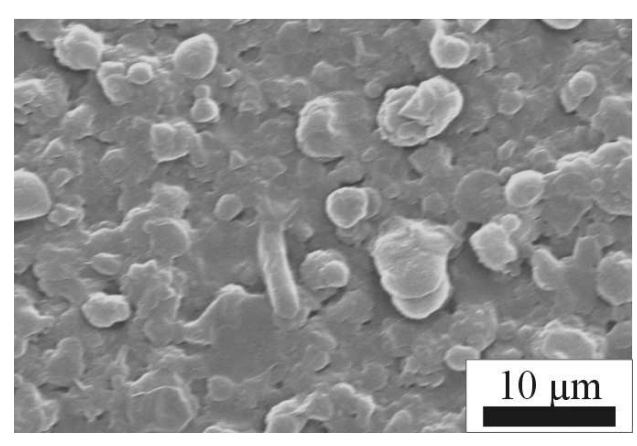

(a) $25{ }^{\circ} \mathrm{C}$

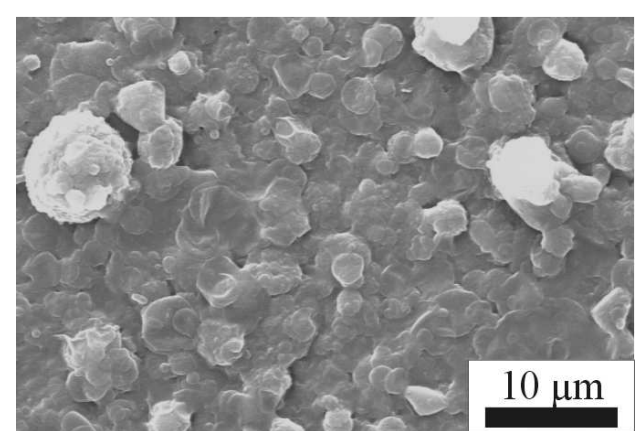

(b) $400{ }^{\circ} \mathrm{C}$

Abbildung 7.7: Rasterelektronenmikroskopische Aufnahmen von PMMASchichten, die bei Raumtemperatur (1500 mJ/ $\mathrm{cm}^{2}$, 5000 Pulse) hergestellt wurden (a), nach Auslagerung für eine Stunde auf $400{ }^{\circ} \mathrm{C}$ (b). Um Aufladungserscheinungen zu vermeiden, wurden die Schichten mit einer dünnen Au-Schicht überzogen. 
mit reaktiven kleinen Degradationsprodukten an der Oberfläche vernetzt werden (vgl. Abb. 4.1), entsteht ein vernetztes Skelett. Infolgedessen bleibt die Mikrostruktur der Filme auch weit oberhalb der Glastemperatur des Polymers $\left(90-100{ }^{\circ} \mathrm{C}\right.$ bei $\mathrm{M}_{n} \simeq 6 \mathrm{~kg} / \mathrm{mol}$, vgl. Kap. 7.1 oder [73]) erhalten, da die Mobilität der Makromoleküle eingeschränkt ist. Indirekt deuten die REM-Aufnahmen von Proben, die bis auf $400{ }^{\circ} \mathrm{C}$ ausgelagert wurden, auf einen schwammähnlichen Film hin, in dem durch den Zerfall des Polymers Poren gebildet werden, ohne dass die Mikrostruktur der Filme signifikante Veränderungen aufweist.

Bei weiterer Erhöhung der Auslagerungstemperatur können auch Bindungen innerhalb der vernetzten Phase aufgebrochen werden. Erst bei Temperaturen von $600{ }^{\circ} \mathrm{C}$ ist kein Rückstand des laserdeponierten Filmes mehr nachweisbar.

Die Auslagerung von laserdeponierten PMMA-Proben weist somit drei unterschiedliche Temperaturbereiche auf: bei moderaten Temperaturen können keine chemischen Veränderungen der Filme nachgewiesen werden. In diesem Temperaturbereich weist der polymere Anteil der Filme einen Glasübergang auf, der entsprechend der Molmasse bei etwa $90{ }^{\circ} \mathrm{C}$ liegt. Oberhalb von $200{ }^{\circ} \mathrm{C}$ zerfällt das Polymer über eine thermische Degradation. Dieser Prozess ist bei $400{ }^{\circ} \mathrm{C}$ abgeschlossen. Übrig bleibt ein Rückstand von etwa 20 Gew.-\%, der auf die vernetzte Komponente der Filme zurückzuführen ist. Eine nachträgliche Auslagerung der Proben bietet damit die Möglichkeit, die Filme zu modifizieren. Technologisch von Interesse ist vor allem der Temperaturbereich bis $200{ }^{\circ} \mathrm{C}$, in dem Relaxationsprozesse im Polymer möglich sind, die chemische Zusammensetzung der Schichten aber nicht verändert wird. Dies sollte sich auf die makroskopischen Eigenschaften der Filme auswirken, wie im Folgenden beispielhaft anhand der mechanischen Eigenschaften gezeigt wird.

\subsection{Mechanische Eigenschaften}

In Abbildung 7.8 ist der reduzierte Elastizitätsmodul $E_{\mathrm{HU}} /\left(1-\nu^{2}\right)$ aus der Nanoindentation von PMMA-Filmen, die bei Raumtemperatur hergestellt wurden, als Funktion der Auslagerungstemperatur dargestellt. Tatsächlich fällt dieser E-Modul mit steigender Auslagerungstemperatur von 3,2 GPa auf etwa 2,2 GPa bei $250{ }^{\circ} \mathrm{C}$ ab. Gleichzeitig nimmt auch die Universalhärte von $64 \mathrm{~N} / \mathrm{mm}^{2}$ auf $45 \mathrm{~N} / \mathrm{mm}^{2}$ ab. Oberhalb von $250{ }^{\circ} \mathrm{C}$ fällt der E-Modul sodann sprunghaft auf etwa $1 \mathrm{GPa}$ bei 


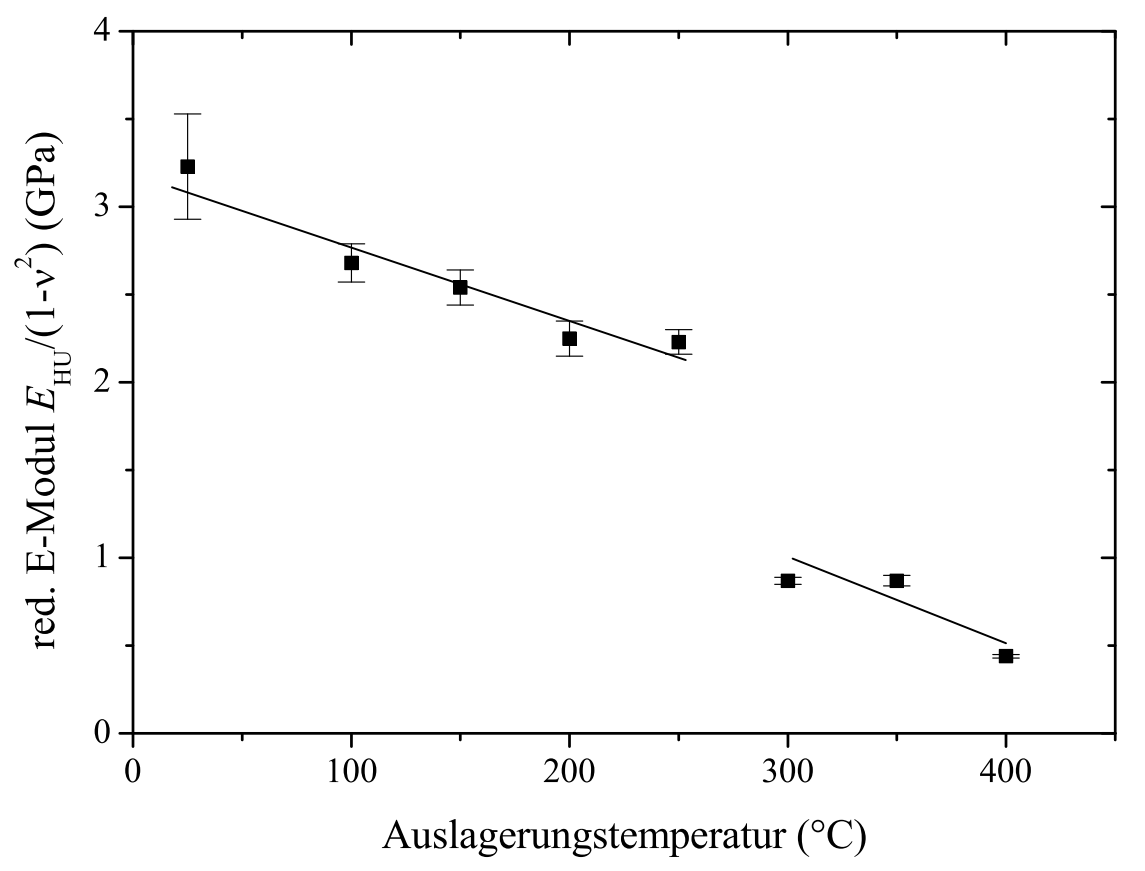

Abbildung 7.8: Reduzierter E-Modul von PMMA-Schichten $\left(1500 \mathrm{~mJ} / \mathrm{cm}^{2}\right.$, 20000 Pulse) als Funktion ihrer Auslagerungstemperatur (gemessen bei Raumtemperatur).

$300{ }^{\circ} \mathrm{C}$.

Der Verlauf des Elastizitätsmoduls lässt sich aus der dielektrischen Spektroskopie und der thermogravimetrischen Analyse erklären: zunächst bleibt das Material bis zu Temperaturen von $215{ }^{\circ} \mathrm{C}$ erhalten. Da auch die chemische Zusammensetzung keine signifikanten Veränderungen aufweisen kann, deutet das Erweichen des Materials in diesem Temperaturbereich auf Relaxationseffekte im Polymer hin, die mit einer steigenden Mobilität des unvernetzten Anteils bei $\alpha$ - und $\beta$-Relaxation einhergehen. Vermutlich damit verbunden ist das Ausheilen von Defekten in den Filmen, in dem die Makromoleküle vom Depositionszustand in einen relaxierteren Zustand übergehen. Solche Relaxationen sind typisch für amorphe Materialien, in denen durch den Abschreckvorgang freies Volumen eingefroren ist. Besonders charakteristisch sind diese Alterungserscheinungen bei Polymeren [90]. Auch bei nanokristallinen laserdeponierten Metallen wurden ähnliche Relaxationseffekte gefunden [88], die auf eine 
Erzeugung von Defekten während des Depositionsvorganges zurückgeführt werden konnten.

Die starke Abnahme des Elastizitätsmoduls bei Erhöhung der Temperatur auf über $250{ }^{\circ} \mathrm{C}$ ist mit einem deutlichen Materialverlust verbunden, der auf den Zerfall des unvernetzten Polymers hindeutet. Der auf etwa 1 GPa gefallene reduzierte Elastizitätsmodul repräsentiert den verbleibenden schwammähnlichen Rückstand.

\subsection{Mechanische Spektroskopie}

Relaxationen im Temperaturbereich bis $250{ }^{\circ} \mathrm{C}$ lassen sich auch mit der mechanischen Spektroskopie charakterisieren, in dem die Probe zyklischen Anregungen ausgesetzt wird. Dazu wurden in Zusammenarbeit mit dem I. Physikalischen Institut (Universität Göttingen) PMMA-Filme direkt auf einen Si-Doppelpaddeloszillator aufgebracht. Diese Oszillatoren werden zu freien, mechanischen Schwingungen angeregt und bei einer Frequenz von $5,7 \mathrm{kHz}$ temperaturabhängig die Dämpfung gemessen. Eine detaillierte Beschreibung dieser Experimente ist bei Rösner [76, 77] zu finden. In Abbildung 7.9 ist ein Dämpfungsspektrum als Differenz der Spektren des beschichteten und unbeschichteten Oszillators dargestellt. Zu erkennen ist - wie in der dielektrischen Spektroskopie (vgl. Abb. 7.1) - ein Maximum in der Dämpfung bei etwa $140{ }^{\circ} \mathrm{C}$. Die Energieaufnahme der Probe in diesem Maximum weist auf die mit dem Glasübergang verbundene $\alpha$-Relaxation und die mit der Drehung der $\mathrm{C}(=\mathrm{O}) \mathrm{OCH}_{3}$-Seitengruppe verbundene $\beta$-Relaxation hin, die bei einer Anregungsfrequenz von 5,4 kHz zusammenfallen [1]. Die unterschiedliche Position der Maxima in der mechanischen und dielektrischen Spektroskopie ist in der unterschiedlichen Art der Anregung begründet: Die dielektrische Spektroskopie wird über eine kapazitive Anregung realisiert, die auf das mit der Nebengruppe $\mathrm{C}(=\mathrm{O}) \mathrm{OCH}_{3}$ verbundene Dipolmoment wirkt. Es wird daher primär die $\beta$-Relaxation des PMMA und erst im zweiten Schritt über die Bewegung der Seitengruppen die $\alpha$-Relaxation des ganzen Makromoleküls angeregt. Anders wird der Anregungsmechanismus bei der mechanischen Spektroskopie verwirklicht, wo durch die mechanische Schwingung eine Bewegung auf die Makromoleküle übertragen wird, und damit stärker die $\alpha$ - als die $\beta$-Relaxation angeregt wird [77].

Für das zweite, in der mechanischen Spektroskopie bei $240{ }^{\circ} \mathrm{C}$ zu erkennende 


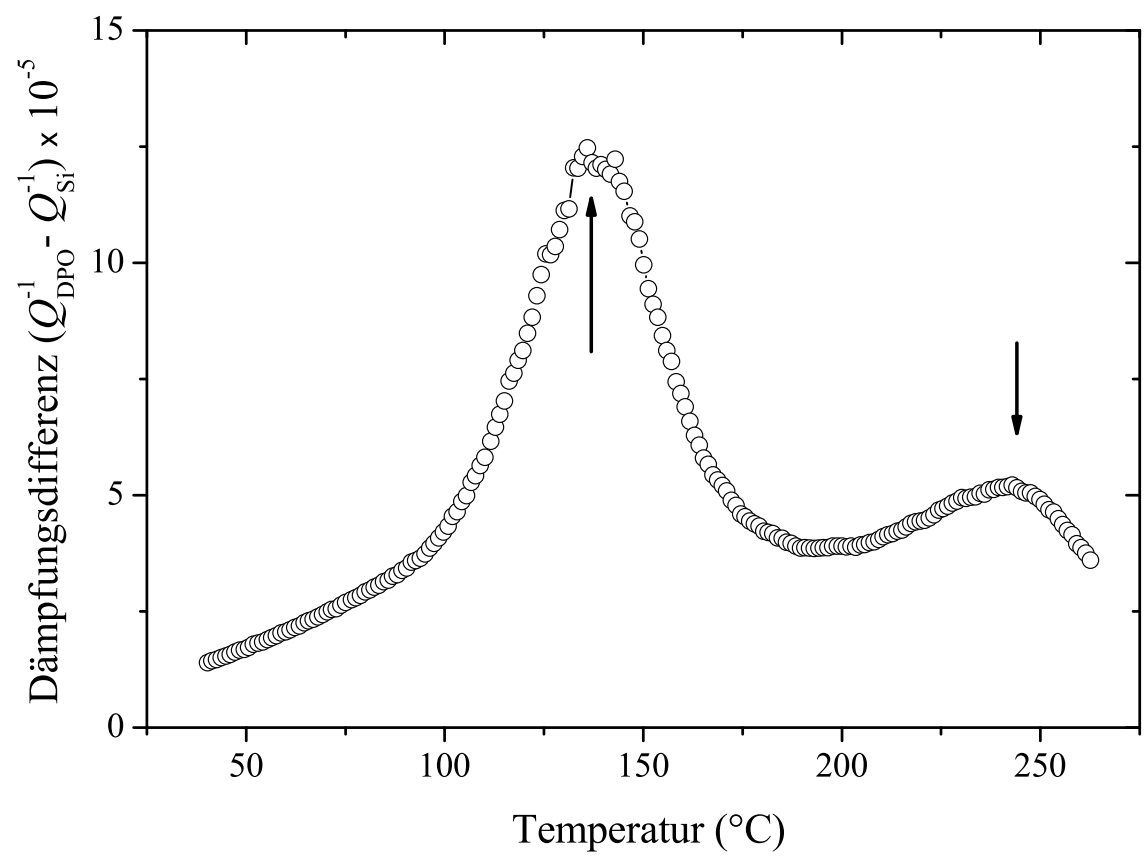

Abbildung 7.9: Dämpfungsspektrum als Differenz von mit PMMA beschichtetem $\left(1500 \mathrm{~mJ} / \mathrm{cm}^{2}, 5000\right.$ Pulse) und unbeschichtetem Oszillator, aufgenommen während eines Heizzyklus [76].

Maximum gibt es in der Literatur keine Entsprechung. Es deutet erneut auf die zweikomponentige Zusammensetzung laserdeponierter PMMA-Filme hin: Der vernetzte Schichtanteil kann über einen der $\alpha$ - oder $\beta$-Relaxation ähnlichen Prozess Energie aufnehmen. Die im Vergleich zu PMMA hohe Relaxationstemperatur ist auf die Vernetzung des Materials zurückzuführen. Vergleichbare Ergebnisse sind auch für vernetztes Poly(vinyl-zimtsäureester) (PVCin) bekannt, das eine mit dem Vernetzungsgrad ansteigende Glastemperatur [43] aufweist. Im Vergleich zum PVCin-System, wo bisher eine maximale Temperaturdifferenz von $30 \mathrm{~K}$ bei einem Vernetzungsgrad von etwa $70 \%$ beobachtet wurde, erscheint der hier beschriebene Anstieg der Relaxationstemperatur um $100 \mathrm{~K}$ recht hoch. Da in diesem Temperaturbereich aber bereits ein nennenswerter Materialverlust auftritt, sind Aussagen über Relaxationen und Umwandlungen in der Probe, durch die mechanische Energie aufgenommen werden kann, nicht mehr eindeutig zu gewährleisten. 
Abbildung 7.10 beschreibt die Relaxation eines laserdeponierten PMMA-Filmes bis $200{ }^{\circ} \mathrm{C}$ : sie zeigt Dämpfungsspektren des Filmes im Bereich der $\alpha$ - und $\beta$ Relaxation, die jeweils eine maximale Energieaufnahme bei etwa $140{ }^{\circ} \mathrm{C}$ aufweisen (vgl. Abb 7.9). Diese maximale Dämpfung steigt mit der Auslagerungstemperatur von $6,5 \cdot 10^{5}$ auf $1,4 \cdot 10^{6}$ an, was auf eine Verfestigung der Probe bei der jeweils maximalen Auslagerungstemperatur zurückzuführen ist (vgl. [76]).

Diese Verfestigung lässt sich mit Hilfe der Frequenzverschiebung des Oszillators quantifizieren. Abbildung 7.11 zeigt die Frequenzverschiebung bei einer festen Temperatur von $40{ }^{\circ} \mathrm{C}$ als Funktion der maximalen Auslagerungstemperatur, die mit zunehmender Temperatur zu größeren negativen Werten ansteigt. Unter der Annahme einer kontinuierlichen Schicht mit konstanter Dicke kann hiermit der Schermodul $G$ aus der Dicke $d$ und Dichte $\rho$ der Schicht und den entsprechenden Konstanten

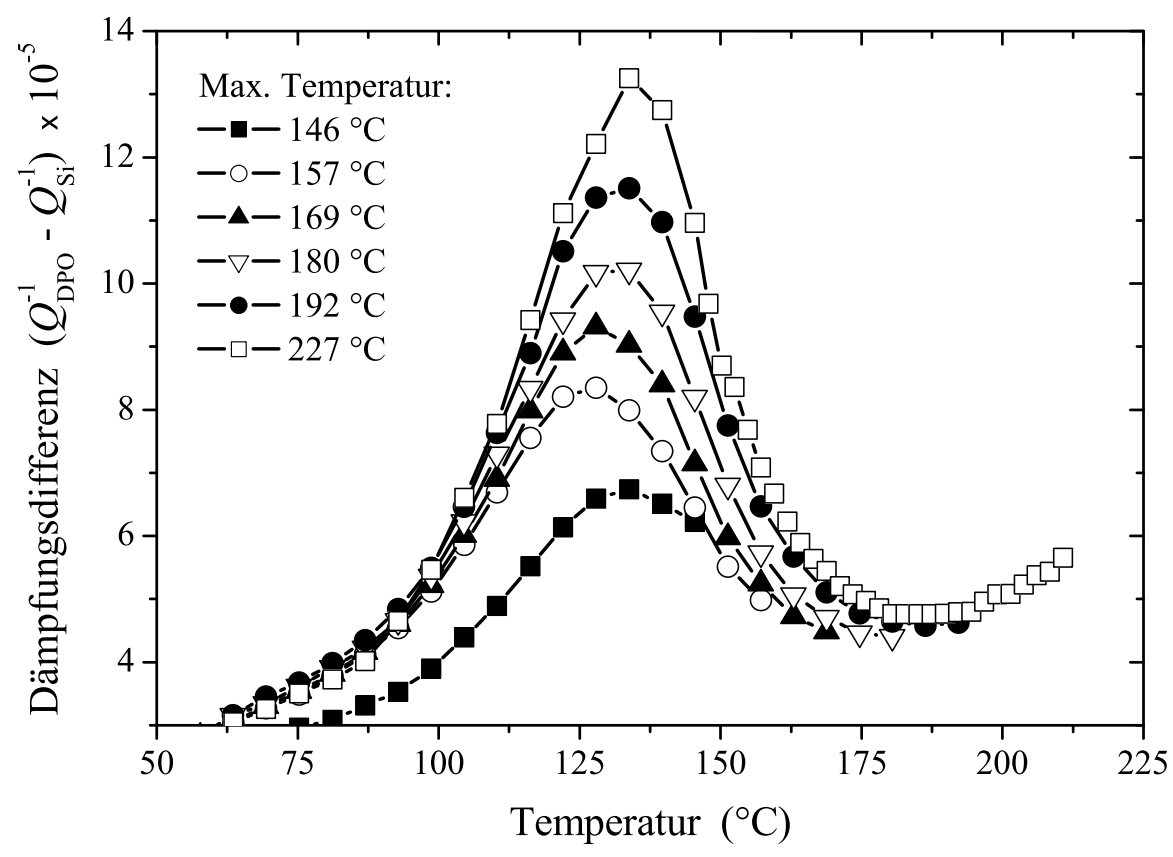

Abbildung 7.10: Differenz der Dämpfung von mit PMMA beschichtetem (1500 mJ $/ \mathrm{cm}^{2}, 5000$ Pulse) und unbeschichtetem Oszillator für verschiedene Heizzyklen mit jeweils steigender maximaler Auslagerungstemperatur (vgl. $[76])$. 
des Si-Substrates über [76]

$$
G_{\text {Film }}=\left(\frac{\Delta f}{f}+\frac{\rho_{\text {Film }} d_{\text {Film }}}{2 \rho_{\mathrm{Si}} d_{\mathrm{Si}}}\right) \cdot \frac{2 G_{\mathrm{Si}} d_{\mathrm{Si}}}{3 d_{\mathrm{Film}}}
$$

bestimmt werden. Entsprechende Daten sind in Abbildung 7.11 (b) aufgetragen. Der so bestimmte Schermodul ist mit 7,4 GPa im Vergleich zu Literaturdaten (1,7 GPa [104]) hoch. Teilweise kann diese Diskrepanz mit Abschätzungsfehlern begründet werden: In den Schermodul geht die Schichtdicke ein, die aus einer typischen Depositionsrate abgeschätzt wurde, und so leicht einen Fehler von 50\% aufweisen kann.

\section{Temperatur $\left({ }^{\circ} \mathrm{C}\right)$}

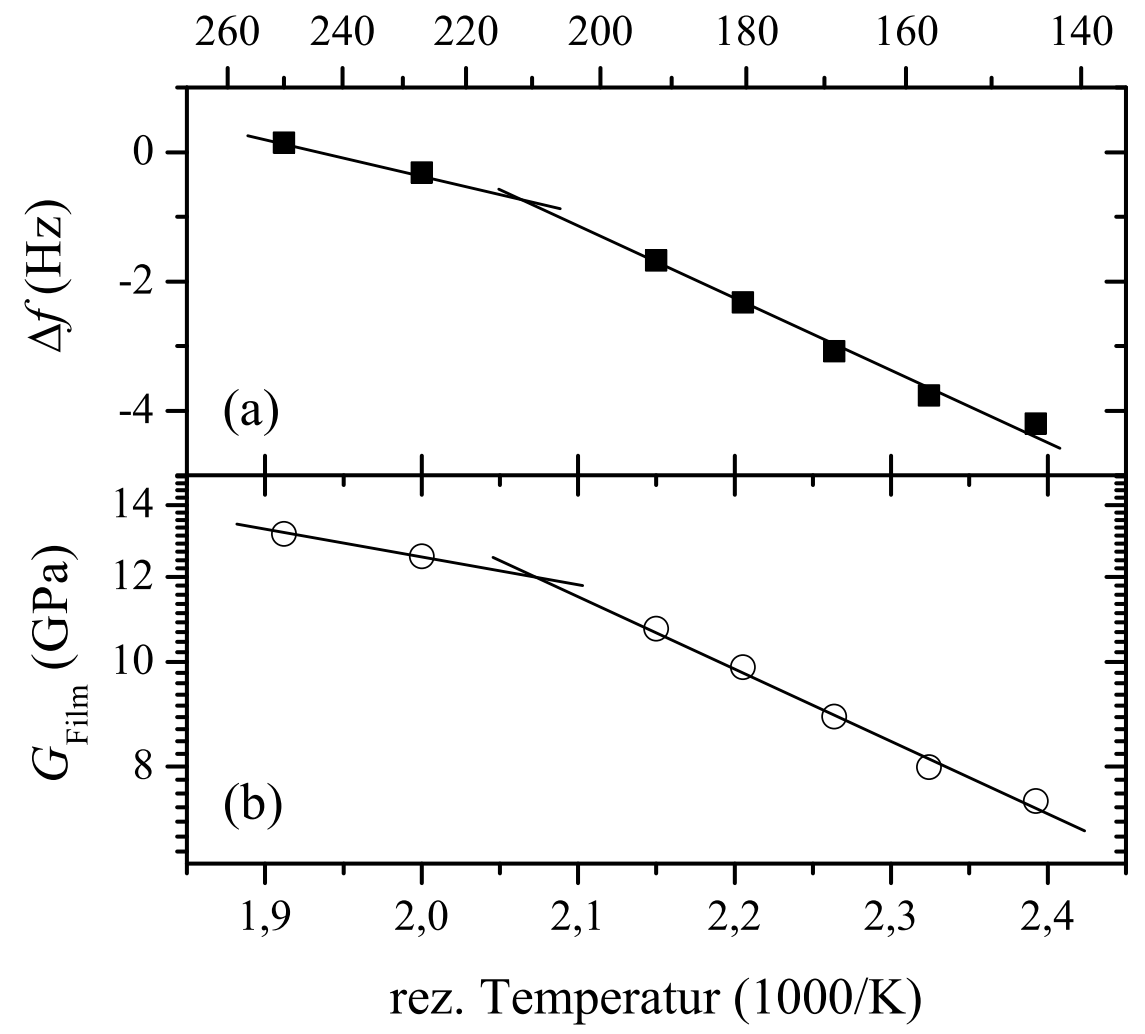

Abbildung 7.11: Frequenzverschiebung $\left(f_{\mathrm{DPO}}-f_{\mathrm{Si}}\right)$ des DPO-Systems mit einer PMMA-Schicht (1500 mJ/ $\mathrm{cm}^{2}, 5000$ Pulse) als Funktion der maximalen Auslagerungstemperatur, gemessen beim jeweiligen Kühlzyklus bei einer Temperatur von $40{ }^{\circ} \mathrm{C}$ (a) und daraus bestimmter Schermodul (b). 
Der absolute Fehler des Schermoduls liegt daher bei 2 GPa, relative Änderungen des Moduls werden durch diese Abschätzungsungenauigkeiten jedoch nicht beeinflusst. Die auch unter Berücksichtigung dieses Fehlers verbliebene Differenz zu Literaturdaten lässt sich analog zur Härte (vgl. Kap. 4.5) durch den im Herstellungsprozess vernetzten Schichtanteil verstehen.

Wie schon aus dem Anstieg des Dämpfungsmaximums (vgl. Abb. 7.10) ersichtlich, zeigen die Filme eine weitere Verfestigung mit steigender Auslagerungstemperatur. Der Schermodul steigt von 7,4 GPa nach einer Auslagerung auf etwa $190{ }^{\circ} \mathrm{C}$ bis auf 10,8 GPa an. Die Verfestigung der Filme deutet - wie die Entwicklung des Elastizitätsmoduls (vgl. Kap. 7.4) - auf die Bedeutung von Alterungseffekten [90] hin: Während die laserdeponierten Filme keine chemischen Veränderungen zeigen, verändern sich die mechanischen Eigenschaften der Filme deutlich. Das Abflachen der Kurven oberhalb dieser Temperaturen kann auf den bereits in der thermogravimetrischen Analyse nachgewiesenen Massenverlust zurückgeführt werden.

Die Ergebnisse von mechanischer Spektroskopie und statischer Nanoindentation scheinen zunächst widersprüchlich: der reduzierte Elastizitätsmodul, bestimmt aus Härtemessungen, fällt mit steigender Auslagerungstemperatur um 30\% ab, der Schermodul steigt im gleichen Temperaturintervall um 45\% an. Obwohl Schermodul, bestimmt aus Torsionsexperimenten, und Elastizitätsmodul, bestimmt aus Zugoder Druckexperimenten, sich in der experimentellen Bestimmung unterscheiden, geben sie ähnliche Materialcharakteristika an. In der experimentellen Untersuchung können kleine Unterschiede auf Grund der zweidimensionalen Geometrie der Probe nicht ausgeschlossen werden, aber der fundamentale Unterschied zwischen den hier beschriebenen Ergebnissen ist auf die Viskoelastizität des PMMA zurückzuführen: Bei Anregung mit niedrigen Frequenzen verhält sich das Polymer viskos und fließt unter Spannung, bei hohen Frequenzen reagiert es mit elastischer Dehnung [46, 47]. Die Frequenzbereiche, in denen hier die elastischen Konstanten der Filme charakterisiert wurden, unterschieden sich recht deutlich: in der mechanischen (und dielektrischen) Spektroskopie wird mit einer Frequenz von 5,4 kHz gearbeitet. Die so untersuchten mikroskopischen Prozesse haben eine typische Zeitdauer von 0,18 ms. Im Gegensatz dazu wird der Elastizitätsmodul aus der Entlastung eines Nanoindenterexperiments bestimmt, wo die experimentelle Dauer bei einigen Sekunden liegt. Beide Experimente sprechen also Prozesse an, die auf anderen Zeitskalen liegen, 
und damit grundsätzlich andere Charakteristika der Filme beschreiben. Scher- und Elastizitätsmodul sind folglich ohne Berücksichtigung der Zeitskalen nicht vergleichbar. Die oberhalb der Glastemperatur beobachteten Relaxationen des PMMA-Filme können also gleichzeitig ein Erweichen des Materials bei niedrigen Frequenzen und eine Verfestigung auf kurzen Zeitskalen bewirken. Zur besseren Vergleichbarkeit bieten sich Experimente an, die andere typische Zeitskalen untersuchen. Eine Möglichkeit liegt dabei in der Vibrating-Rheed-Methode mit typischen Anregungsfrequenzen von 500-3000 Hz oder der Dynamisch-Mechanischen Analyse (DMA) mit Schwingungsfrequenzen von 50 bis $500 \mathrm{~Hz}$ (vgl. z.B. [100]). Zur Untersuchung der mikroskopischen Veränderungen der Filme bieten sich ebenfalls weitere Experimente an. Eine Möglichkeit, einen Alterungsprozess zu realisieren, besteht beispielsweise in einer mit einem Ausheilen von freiem Volumen verbundenen mikrostrukturellen Modifikation der Filme. Die dann mit einer Auslagerung verbundene Kompaktierung der Filme sollte in Experimenten zur Bestimmung des freien Volumens sichtbar werden.

Mit den hier verwendeten Methoden konnte somit gezeigt werden, dass laserdeponierte PMMA-Filme bis $\mathrm{zu} 200{ }^{\circ} \mathrm{C}$ thermisch stabil sind und die chemische Zusammensetzung sich nicht verändert. In diesem Temperaturbereich ändern sich die Eigenschaften der Filme, bedingt durch den kalorischen Glasübergang, der wegen der reduzierten Molmasse der Filme um $20 \mathrm{~K}$ vermindert ist. Die mechanischen Eigenschaften der Filme oberhalb des Glasübergangs entwickeln sich kontrovers: Während der Elastizitätsmodul bei niedrigen Untersuchungsfrequenzen abfällt, steigt der Schermodul, bestimmt bei einer Frequenz von 5,4 kHz, an. Auslagerungsprozesse in diesem Temperaturintervall bieten somit eine Möglichkeit, die Charakteristika der Filme nachträglich gezielt zu verändern. Oberhalb dieser Temperaturen setzt ein Zerfall der Filme ein, der auf Grund der zweikomponentigen Zusammensetzung der laserdeponierten PMMA-Filme in zwei Stufen abläuft. Zunächst werden die polymeren Bestandteile entfernt. Der vernetzte Anteil der Filme ist jedoch thermisch deutlich stabiler, so dass ein schwammartiges Material mit einem vernetzten Skelett entsteht, aus dem die unvernetzten Bestandteile weitestgehend entfernt werden und so Poren im Material zurückbleiben. Der Zerfall des vernetzten Materials setzt bei $400{ }^{\circ} \mathrm{C}$ ein und ist oberhalb von $600{ }^{\circ} \mathrm{C}$ abgeschlossen. 


\section{8}

\section{Zusammenfassung}

Ziel dieser Arbeit war, eine zusammenhängende Beschreibung der gepulsten Laserdeposition von PMMA zu erarbeiten. Über eine Charakterisierung der Filme und ein Verständnis der Depositionsmechanismen - unter Berücksichtigung der Ablationsvorgänge - sollte eine systematische Modifikation und Einstellung der Filmeigenschaften möglich werden. Anwendungsbezogen sollten dabei - neben einem Vergleich der laserdeponierten Filme mit anderen typischen Polymerdepositionsverfahren - die makroskopischen mechanischen Eigenschaften der Filme ebenso berücksichtigt werden wie eine nachträgliche Modifikationen der Filme durch thermische Alterung.

Ein besonderes Charakteristikum laserdeponierter PMMA-Filme ist ihre - im Rahmen dieser Arbeit erstmals diskutierte - zweikomponentige Zusammensetzung aus einem Polymer mit einer Molmasse von etwa $8000 \mathrm{~g} / \mathrm{mol}$ und einer vernetzten Komponente. Diese Zusammensetzung spiegelt sich in allen Eigenschaften der Filme - angefangen von der Mikrostruktur über die mechanischen Eigenschaften bis zu den thermischen Veränderungen - wider. Diese zweikomponentige Zusammensetzung der Filme ist auf den Ablationsprozess zurückzuführen, während dessen PMMA chemisch modifiziert wird: Abgetragen werden Polymertröpfchen (Droplets) und kleine Degradationsprodukte, die mit Hilfe eines Massenspektrometers identifiziert werden konnten. Ein wesentlicher Bestandteil dieser Produkte sind Monomere, die bei der Ablation von PMMA zu etwa $40 \%$ gebildet werden. Die übrigen Produkte sind durch die Abspaltung von Teilen der Estergruppe verständlich.

Die zweikomponentige Zusammensetzung bleibt auch während der Deposition 
erhalten. Der Depositionsmechanismus bei Raumtemperatur umfasst neben einem Erstarren der Droplets eine chemische Reaktion, in der aus kleinen Degradationsprodukten eine vernetzte Schicht gebildet wird. Mit Hilfe der Infrarotspektroskopie konnte nachgewiesen werden, dass die vernetzte Komponente - in Übereinstimmung mit den Degradationsprodukten - über eine mit der Estergruppe verbundene Reaktion gebildet wird. Untersuchungen der Filme zeigen die unterschiedlichen Komponenten deutlich: Mikrostrukturelle Charakterisierungen wie Rasterelektronen- und Rasterkraftmikroskopie belegen, dass etwa 60-70\% der Schicht aus Droplets besteht, die die Eigenschaften der Filme wesentlich prägen. Ihre chemische Zusammensetzung entspricht in IR-Spektren einem nur leicht modifizierten PMMA, das eine Molmasse von etwa $8000 \mathrm{~g} / \mathrm{mol}$ aufweist. Neben diesem polymeren Anteil liegen Oligomere vor, die etwa 15 Gew.-\% der Schicht ausmachen, und die auf chemische Reaktionen von Monomeren zurückzuführen sind.

Aus dem Ablations- und Depositionsmechanismus lassen sich Möglichkeiten ableiten, die Eigenschaften der Filme über die Zusammensetzung der Komponenten zu verändern. Zunächst wurde ein Eingriff in den Ablationsprozess vorgeschlagen: Durch eine Variation der Energiedichte werden bei der Ablation vermehrt kleine Produkte freigesetzt. Dadurch sinkt der Anteil der polymeren Komponente an der Schicht. Diese Verschiebung der Zusammensetzung konnte mit Hilfe der IRSpektroskopie genauso wie mit der Größenausschlusschromatographie nachgewiesen werden.

Eine andere Möglichkeit, die Schichteigenschaften zu verändern, bietet eine Modifikation der Ablationsprodukte auf dem Substrat. Daher wurde der Einfluss einer zunehmenden Substrattemperatur auf die Filme charakterisiert. Die zusätzlich auf die Ablationsprodukte übertragene Energie beeinflusst die polymere Komponente wesentlich: Mit der Erhöhung der Mobilität der Makromoleküle verbunden ist eine Glättung der Schicht durch ein Auseinanderfließen der Droplets auf dem Substrat. Zusätzlich zu dieser mikrostrukturellen Veränderung der Filme wächst bei einer Deposition oberhalb von $125{ }^{\circ} \mathrm{C}$ die Molmasse des Polymers bis auf $3,2 \cdot 10^{4} \mathrm{~g} / \mathrm{mol}$ bei $250{ }^{\circ} \mathrm{C}$. Als dominanter Mechanismus kann aus der IR-Spektroskopie eine radikalische Polymerisation von Monomeren ausgeschlossen werden. Vielmehr erscheint auf Grund der Zusammensetzung der Ablationsprodukte eine Reaktion zwischen Makromolekülen wahrscheinlich. Auch die Zusammensetzung der vernetzten Komponente 
wird durch den Einbau hochmolekularen Materials verändert.

Sodann wurden die Veränderungen der Filme bei einer thermischen Auslagerung diskutiert. Wegen der charakteristischen Zusammensetzung lassen sich diese Veränderungen in drei unterschiedliche Bereiche einteilen: bei Auslagerungstemperaturen bis zu $200{ }^{\circ} \mathrm{C}$ sind die Filme chemisch stabil. Dieser Temperaturbereich wird geprägt vom kalorischen Glasübergang der Filme bei etwa $90{ }^{\circ} \mathrm{C}$, der mit Hilfe der dielektrischen Spektroskopie untersucht werden konnte. In diesem Temperaturbereich werden die mechanischen Eigenschaften durch Relaxationsprozesse modifiziert: Durch die Viskoelastizität des Polymers zeigt der mit Hilfe von Nanoindentationsmessungen charakterisierte reduzierte Elastizitätsmodul ein Erweichen der Filme, während der Schermodul, der mit der mechanischen Spektroskopie untersucht wurde, eine Verfestigung des Materials zeigt. Der Temperaturbereich zwischen 200 und $400{ }^{\circ} \mathrm{C}$ ist durch einen Zerfall der polymeren Komponente gekennzeichnet. Dieser Zerfall, der über thermogravimetrische Messungen und die IR-Spektroskopie beschrieben werden konnte, beruht auf der thermischen Degradation von PMMA. Bei Temperaturen oberhalb von $400{ }^{\circ} \mathrm{C}$ kann ein Rückstand von etwa 20 Gew.- $\%$ des Materials nachgewiesen werden, der auf die vernetzte Komponente der laserdeponierten Filme hindeutet. Dieser Rückstand zerfällt im Temperaturintervall von 400 bis $600{ }^{\circ} \mathrm{C}$.

Die makroskopischen mechanischen Eigenschaften werden wesentlich durch die charakteristische Zusammensetzung der Filme geprägt. Der reduzierte Elastizitätsmodul ist mit 3,3 GPa vergleichbar mit den Modulen von idealem PMMA. Als Kontrast dazu weisen die laserdeponierten Filme eine um einen Faktor 5 auf etwa $60 \mathrm{~N} / \mathrm{mm}^{2}$ erhöhte Härte auf, was den Einfluss der vernetzten Komponente auf die mechanischen Eigenschaften zeigt. Auf Grund dieser Abhängigkeiten können auch die mechanischen Eigenschaften der Filme über die Zusammensetzung verändert werden: der wachsende Einfluss der vernetzten Komponente mit steigender Energiedichte führt ebenso zu einer Verfestigung der Filme wie die Zunahme der Molmasse über eine erhöhte Substrattemperatur. Wie für amorphe Materialien typisch, hängen die mechanischen Eigenschaften der Filme zusätzlich von der Vorgeschichte ab: Nach dem Abschreckprozesses liegen die Filme in einer energetisch ungünstigen Materialkonfiguration vor. Durch Alterungsprozesse bietet sich daher die Möglichkeit, die mechanischen Eigenschaften der Filme nachträglich zu verändern. 
Insgesamt konnte mit den durchgeführten Experimenten erstmals eine Beschreibung der gepulsten Laserdeposition von PMMA erstellt werden, die die wesentlichen Eigenschaften der Filme erklärt. Diese weisen eine charakteristische Zusammensetzung aus vernetzter Schicht und polymerartigen Droplets auf, die durch die Depositionsparamter in Grenzen variiert werden kann. Die Eigenschaften der Filme werden von beiden Komponenten geprägt, so dass die gepulste Laserdeposition die Herstellung von Polymerfilmen ermöglicht, die gegenüber den Ausgangsmaterialien geänderte Charakteristika aufweisen. Sie liegen zwischen denen unvernetzten Polymers, wie es sich etwa durch einen Spincoating-Prozess aufbringen lässt, und hochgradig vernetzten Filmen, die durch eine Plasmapolymerisation hergestellt werden können. 


\section{Literaturverzeichnis}

[1] R. Bergman, F. Alvarez, A. Alegria, J. Colmenero, J. Chem. Phys. 109, 7546 (1998)

[2] H. H. Behncke, W. Weiler, Materialprüf. 30, 239 (1988)

[3] H. H. Behncke, Zeitschrift für Wärmebehandlung und Werkstofftechnik 5, 3 (1993)

[4] H. H. Behncke, VDI Berichte 1194, 33 (1995)

[5] H. Biedermann, Y. Osada, Plasma Polymerisation Processes, Elsevier, Amsterdam (1992)

[6] G. Binnig, C. F. Quate, C. Gerber, Phys. Rev. Lett. 56930 (1986)

[7] G. B. Blanchet, Appl. Phys. Lett. 62, 479 (1993)

[8] G. B. Blanchet, S. I. Shah, Appl. Phys. Lett. 62, 1026 (1993)

[9] G. B. Blanchet, Macromolecules 28, 4603 (1995)

[10] G. B. Blanchet, J. Appl. Phys. 80, 4082 (1996)

[11] G. B. Blanchet, P. Cotts, C. R. Fincher, J. Appl. Phys. 88, 2975 (2000)

[12] I. M. Campbell, Introduction to synthetic polymers, Oxford University Press, Oxford (1994)

[13] D. B. Chrisey, G. K. Hubler (editors), Pulsed Laser Deposition Of Thin Films, Wiley Interscience Publication, New York (1994)

[14] D. B. Chrisey, A. Piqué, R. A. McGill, J. S. Horwitz, B. R. Ringeiesen, D. M. Bubb, P. K. Wu, Chem. Rev. 103, 553 (2003)

[15] M. M. Coleman, Y. Hu, M. Sobkowiak, P. C. Painter, J. Pol. Sci. B 36, 1579 (1998)

[16] R. Cristescu, G. Sobol, I. N. Mihailescu, M. Popescu, F. Sava, E. Ion, C. O. Morosnu, I. Stamatin, Appl. Surf. Sci. 208-209, 645 (2003)

[17] B. D. Cullity (editor), Elements of X-ray Diffraction, Addison-Wesley Publishing Company, Massachusetts (1967)

[18] B. Danielzik, N. Fabricius, M. Röwekamp, D. von der Linde, Appl. Phys. Lett. 48, $212(1986)$ 
[19] G. M. Davis, M. C. Gower, C. Fortakis, T. Efthimiopoulos, P. Argyrakis, Appl. Phys. A 36, $27(1985)$

[20] J. Dechant, Ultrarotspektroskopische Untersuchung an Polymeren, Akademie Verlag, Berlin (1972)

[21] D. Dijkkamp, T. Venkatesan, X. D. Wu, S. A. Shareen, N. Jiswari, Y. H. Min-Lee, W. L. McLean, M. Croft, Appl. Phys. Lett. 51, 619 (1987)

[22] P. Dyer, R. Srinivasan, J. Appl. Phys. 66, 2608 (1989)

[23] P. E. Dyer, Appl. Phys. A 77, 167 (2003)

[24] T. Efthimiopoulos, C. Kiagias, G. Heliotis, E. Helidonis, Can. J. Phys. 78, 509 (2000)

[25] H. G. Elias, Makromoleküle, Hüthig \& Wepf, Basel (1992)

[26] R. C. Estler, N. S. Nogar, Appl. Phys. Lett. 49, 1175 (1986)

[27] S. Fähler, H.-U. Krebs, Appl. Surf. Sci. 96-98, 61 (1996)

[28] S. Fähler, M. Strömer, H.-U. Krebs, Appl. Surf. Sci. 109/110, 433 (1997)

[29] S. Fähler, Dissertation, Universität Göttingen (1998)

[30] D. Fleischer, Physical Properties of Monomers, in Polymer Handbook, Hrsg. J. Brandrup, E. H. Immergut, Wiley, New York (1989)

[31] J. A. Forrest, K. Dalnoki-Verres, J. R. Dutcher, Phys. Rev. E 56, 5705 (1997)

[32] J. A. Forrest, K. Dalnoki-Verres, Adv. Col. Int. Sci. 94, 167 (2001)

[33] H. Fujiwara, T. Hayashi, H. Fukumara, H. Masuhara, Appl. Phys. Lett. 64, 2451 (1994)

[34] B. J. Garrison, R. Srinivasan, Appl. Phys. Lett. 44, 849 (1984)

[35] D. B. Geohegan, Appl. Phys. Lett. 63, 1463 (1993)

[36] A. Gibaud, S. Hazra, Current Science 78, 1467

[37] P. Gotthardt, A. Grüger, H. G. Brion, R. Plaetschke, R. Kirchheim, Macromolecules 30, 8058 (1997)

[38] H. Günzler, H. M. Heise, IR-Spektroskopie, VCH-Verlag, Weinheim (1996)

[39] A. Gupta, R. Liang, F. T. Tsay, J. Moacanin, Macromolecules 13, 1696 (1980)

[40] J. Hachenberg, C. Streng, E. Süske, S. Vauth, S. G. Mayr, H.-U. Krebs, K. Samwer, Phys. Rev. Lett. 92, 246102 (2004)

[41] S. G. Hansen, T. E. Robitaille, A. Appl. Lett. 52, 81 (1988)

[42] S. G. Hansen, T. E. Robitaille, J. Appl. Phys. 64, 2122 (1988) 
[43] T. Haramina, private Mitteilung (2005)

[44] J. Heitz, J. T. Dickinson, Appl. Phys. A 68, 515 (1999)

[45] B. L. Henke, E. M. Gullikson, J. C. Davis, Atomic Data and Nuclear Data Tables 54, 181 (1993), in: Atomic scattering factors: X-Ray interaction with Matter, http://wwwcxro.lbl.gov/optical_constants/

[46] Physik der Polymere, 22. IFF-Ferienkurs, Forschungszentrum Jülich (1991)

[47] Soft Matter, 33. IFF-Ferienkurs, Reihe Materie und Material 10, Forschungszentrum Jülich (2002)

[48] H. H. G. Jellinek, M. D. Luh, Makromol. Chem. 115, 89 (1968)

[49] J. L. Keddie, R. A. L. Jones, R. A. Cory, Europhys. Lett. 27, 59 (1994)

[50] J. L. Keddie, R. A. L. Jones, R. A. Cory, Faraday Discuss. 98, 219 (1994)

[51] R. Kelly, J. E. Rothenberg, Nucl. Instr. Meth. B 7/8, 755 (1985)

[52] R. Kelly, J. J. Cuomo, P. A. Leary, J. E. Rothenberg, B. E. Braren, C. F. Aliotta, Nucl. Instr. Meth. B 9, 329 (1985)

[53] R. Kelly, A. Miotello, B. Braren, A. Gupta, K. Casey, Nucl. Instr. Meth. B 65, 187 (1992)

[54] K. Konstadinidis, B. Thakkar, A. Chakraborty, L.W. Potts, R. Tannenbaum, M. Tirrell, Langmuir 8, 1307 (1992)

[55] H.-U. Krebs, O. Bremert, Appl. Phys. Lett. 62, 2341 (1993)

[56] H.-U. Krebs, Int. J. Non-Equilib. Proc. 10, 3 (1997)

[57] S. Küper, M. Stuke, Appl. Phys. B 44, 199 (1987)

[58] S. Küper, M. Stuke, Appl. Phys. A 49, 211 (1989)

[59] S. Küper, S. Modaressi, M. Stuke, J. Phys. Chem. 94, 7514 (1990)

[60] R. Larciprete, M. Stuke, Appl. Phys. B 42, 181 (1987)

[61] S. Lazare, W. Guan, D. Drillhole, Appl. Surf. Sci. 96-98, 605 (1996)

[62] S. Lazare, J. Lopez, J.-M. Turlet, M. Kufner, S. Kufner, P. Chavel, Appl. Opt. 35, 4471 (1996)

[63] S. Lazare, J. Lopez, F. Weisbuch, Appl. Phys. A 69, S1 (1999)

[64] T. Lippert, R. L. Webb, S. C. Langford, J. T. Dickinson, J. Appl. Phys. 85, 1838 (1999)

[65] D. Lubben, S. A. Barnett, K. Susuki, S. Gorbatkin, J. E. Greene, J. Vac. Sci. Technol. B 3, 968 (1985) 
[66] J. G. Lunney, Appl. Surf. Sci. 55, 79 (1995)

[67] H. Lüth, Surfaces and Interfaces of Solid Materials, Springer, Berlin (1995)

[68] L. E. Manring, Macromolecules 24, 3304 (1991)

[69] M. Matsuguchi, J. Minra, Y. Sakai, J. Pol. Sci. 78, 1744 (2000)

[70] A. Miotello, R. Kelly, Appl. Phys. Lett. 67, 3535 (1995)

[71] C. Mortimer, Chemie, Georg Thieme Verlag, Stuttgart (1987)

[72] T. Novet, S. Kevan, D. C. Johnson, Mat. Sci. Eng. A 195, 21 (1995)

[73] K. O’Driscoll, R. Amin Sanyei, Macromolecules 24, 4479 (1991)

[74] W. C. Oliver, G. M. Pharr, J. Mater. Res. 7, 1564 (1992)

[75] L. G. Parrat, Phys. Rev. 95, 359 (1954)

[76] P. Rösner, Dissertation, Universität Göttingen (2004)

[77] P. Rösner, J. Hachenberg, K. Samwer, R. Wehn, P. Lunkenheimer, A. Loidl, E. Süske, T. Scharf, H.-U. Krebs, Phys. Rev. B, (eingereicht 2004)

[78] C. Scheer, J. M. Olaf, E. Schenuit, Materialprüfung 10 (1993)

[79] H. Schmidt, J. Ihlemann, B. Wolff-Rottke, K. Luther, J. Troe, J. Appl. Phys. 83, 5458 (1998)

[80] C. Shim, D. Jung, Jpn. J. Appl. Phys. 43, 940 (2004)

[81] H. M. Smith, A. F. Turner, Appl. Opt. 4, 147 (1965)

[82] R. Srinivasan, B. Braren, D. E. Seeger, R. W. Dreyfus, Macromolecules 19, 916 (1985)

[83] R. Srinivasan, B. Braren, R. W. Dreyfus, L. Hadel, D. E. Seeger, J. Opt. Soc. Am. B. 3, 785 (1986)

[84] R. Srinivasan, B. Braren, K. G. Casey, M. Yeh, Appl. Phys. Lett 55, 2790 (1989)

[85] R. Srinivasan, B. Braren, K. G. Casey, J. Appl. Phys. 68, 1842 (1990)

[86] R. Srinivasan, J. Appl. Phys. 73, 2743 (1993)

[87] R. Srinivasan, Appl. Phys. A 56, 417 (1993)

[88] M. Störmer, Dissertation, Universität Göttingen (1998)

[89] K. Sturm, Dissertation, Universität Göttingen (2000)

[90] L. C. E. Struik, Physical Aging im Amorphous Glassy Polymers and other Materials, Elsevier, Amsterdam (1978) 
[91] E. Süske, Diplomarbeit, Universität Göttingen (2001)

[92] E. Süske, T. Scharf, P. Schaaf, E. Panchenko, D. Nelke, M. Buback, H. Kijweski, H.-U. Krebs, Appl. Phys. A 79, 1295 (2004)

[93] E. Sutcliff, R. Srinivasan, J. Appl. Phys. 60, 3315 (1986)

[94] R. Tannenbaum, C. Hakanson, A. Zeno, M. Tirrell, Langmuir 18, 5592 (2002)

[95] Y. Tsuboi, M. Goto, A. Itaya, J. Appl. Phys. 95, 4189 (1999)

[96] Y. Tsuboi, H. Adachi, E. Yamamoto, A. Itaya, Jpn. J. Appl. Phys. 41, 885 (2002)

[97] M. Tsunekawa, S. Nishio, H. Sato, J. Appl. Phys. 76, 5598 (1994)

[98] A. A. Voevedin, S. J. Laube, S. D. Walck, J. S. Solomon, M. S. Donley, J. S. Zabinski, J. Appl. Phys. 78, 4123 (1995)

[99] W. E. Wallace, J. H. van Zanten, W. L. Wu, Phys. Rev. E 52, R3329 (1995)

[100] I. M. Ward, Mechanical Properties of Solid Polymers, Wiley, Chichester (1983)

[101] S. W. Wee, S. M. Park, Bull. Korean Chem. 22, 914 (2001)

[102] H. A. Willis, V. J. I. Zichy, P. J. Hendra, Polymer 10, 737 (1969)

[103] D. L. Windt, IMD 4.4.1, http://cletus.phys.columbia.edu/ windt/imd/ (2000)

[104] W. Wunderlich, Physical Constants of Poly(methyl methacrylate), in Polymer Handbook, Hrsg. J. Brandrup, E. H. Immergut, Wiley, New York (1989)

[105] J. H. van Zanten, W. E. Wallace, W. Wu, Phys. Rev. E 53, R2053 (1996) 


\section{Danke schön ...}

... allen, die zum Gelingen dieser Arbeit beigetragen haben - sei es durch fachliche oder technische Hilfe, sei es durch fachfremde und zwischenmenschliche Unterstützung ...

- Beginnen möchte ich mit Prof. Dr. Krebs, bei dem ich mich ganz herzlich bedanken möchte. Er hat diese Arbeit erst ermöglicht und durch sein stetes Interesse und sei-

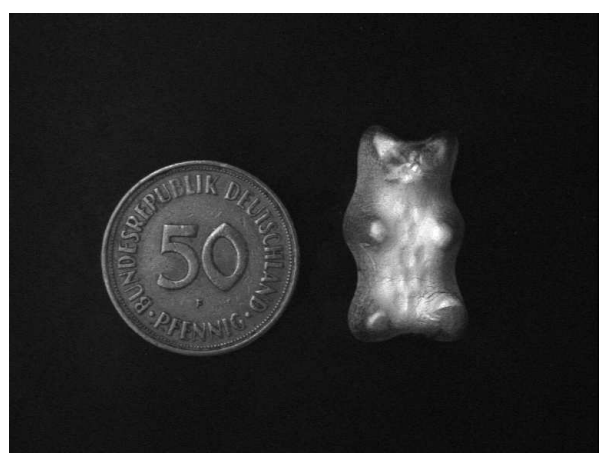
ne große Diskussionsbereitschaft einen wesentlichen Anteil daran. Nennenswert ist auch die besondere Atmosphäre, die die Physik in der Lasergruppe sehr aufgelockert hat. Mein besonderer Dank gilt daher auch der Gruppe mit assimilierten Mitgliedern, Thorsten Scharf, Jörg Faupel, Andreas Meschede, Johanna Röder, Carsten Herweg und Conni Mewes, die beim wöchentlichen Schweinemarmeladenkonsum die Diskussion nicht zu kurz haben kommen lassen. Nicht vergessen möchte ich auch die Ehemaligen Prof. Dr. Satish Vitta, Kai Sturm, Martin Weisheit und Christian Fuhse.

- Herrn Prof. Dr. Kirchheim danke ich für die große Unterstützung innerhalb des SFBs, und der Gruppe Polymerphysik - allen voran Tatjana Haramina für die Zusammenarbeit mit Poly(vinyl-zimtsäureester).

- Eine große Bereicherung war das von Prof. Dr. Suhm vorbildlich organisierte Graduiertenkolleg 782, das durch seine angenehme Diskussionsatmosphäre viel zum Verständnis chemischer Vorgänge beigetragen hat. In diesem Rahmen besonders hervorzuheben ist die Polymerabteilung von Prof. Dr. Buback am 
Institut für Physikalische Chemie mit Dorit Nelke, Elena Panchenko und vor allem Thomas Junkers und Mark Egorov, die neben der Unterstützung mit der Größenausschlusschromatographie noch viele Anregungen parat hatten.

- Besonders bedanken möchte ich mich auch beim I. Physikalischen Institut für die gute Zusammenarbeit: Peter Rösner steuerte neben den DPO-Analysen nicht nur die Kontakte nach Augsburg bei, sondern hat durch sein Paddeln auch manches Seminar aufgelockert ..., wohingegen die AFM-Messungen dieser Arbeit in Zusammenarbeit mit Christian Vree und Jörg Hachenberg entstanden sind. An dieser Stelle nicht unerwähnt bleiben sollen die dielektrischen Verlustspektren, die von Prof. Dr. Loidl, PD Dr. Lunkenheimer und Robert Wehn, Institut für Experimentalphysik V, Universität Augsburg, aufgenommen wurden.

- Mein Dank gilt auch PD Dr. Dr. Kijewski, Institut für Rechtsmedizin, und Prof. Dr. P. Schaaf am II. Phys. Institut für die Bereitstellung des FTIRSpektrometers bzw. des Nanoindenters, sowie Dr. Sebastian Kranzusch am Laserlaboratorium Göttingen e.V. für die Transmissionsmessungen.

- Bedanken möchte ich mich auch bei allen Mitarbeitern des Instituts für Materialphysik für das - nicht nur im zweiten Stock - angenehme Arbeitsklima. Außerdem schulde ich allen technischen Mitarbeitern des Instituts und der Werkstatt besonderen Dank nicht nur für die große Hilfe beim Umzug und Wiederaufbau ...

- Bei allen meinen Freunden möchte ich mich für die vielen, schönen gemeinsamen Stunden bedanken, in denen sie mich an die wesentlichen Aspekte des Lebens neben der Physik erinnert haben.

- Herzlich bedanken möchte ich mich auch bei meinen Eltern, die mir dieses Studium ermöglicht und mich dabei immer unterstützt haben.

- Nicht vergessen möchte ich auch Dorthe und Indra, die immer für eine Überraschung gut waren und wesentlich zu meiner Ablenkung beigetragen haben. 


\section{Lebenslauf}

\section{Persönliche Daten}

$\begin{array}{ll}\text { Name: } & \text { Erik Süske } \\ \text { Geburtsdaten: } & \text { 20.04.1975 in Hameln } \\ \text { Staatsangehörigkeit: } & \text { deutsch }\end{array}$

\section{Schulbildung}

$\begin{array}{ll}1981-1985 & \text { Grundschule Hehlen } \\ 1985-1986 & \text { Orientierungsstufe Bodenwerder } \\ 1986-1994 & \text { Viktoria-Luise-Gymnasium Hameln } \\ 10.06 .1994 & \text { Abitur }\end{array}$

\section{Studium}

WS 1995

17.10.1997

WS 1997

SS 1998

WS 1998-SS 2001

11.05.2001

SS 2002

WS 2002/2003
Aufnahme des Physikstudium an der Universität Göttingen Vordiplomprüfung Physik

Hauptstudium Physik an der Universität Göttingen Erasmusstipendium Physik an der Universität Groningen, Niederlande Hauptstudium Physik an der Universität Göttingen Diplomarbeit bei Prof. Dr. H.-U. Krebs, Institut für Materialphysik zum Thema Laserdeposition von Polymer-Metall-Schichten Diplom

Einschreibung in den Aufbaustudiengang Physik an der Universität Göttingen Umschreibung in den Promotionsstudiengang Physik

\section{Beschäftigungen}

